\title{
LEVI-FLAT INVARIANT SETS OF HOLOMORPHIC SYMPLECTIC MAPPINGS
}

\author{
by Xianghong GONG
}

\section{Introduction.}

Let $\varphi$ be a biholomorphic mapping defined near the origin of $\mathbb{C}^{2 n}$ with $\varphi(0)=0$. Recall that $\varphi$ is symplectic if it preserves the holomorphic symplectic 2-form $\sum_{j=1}^{n} d \xi_{j} \wedge d \eta_{j}$. Assume that the linear part of $\varphi$ is diagonalizable. In suitable local holomorphic symplectic coordinates, $\varphi$ is then given by

$$
\xi_{j} \rightarrow \lambda_{j} \xi_{j}+u_{j}(\xi, \eta), \quad \eta_{j} \rightarrow \lambda_{j}^{-1} \eta_{j}+v_{j}(\xi, \eta), \quad j=1, \ldots, n,
$$

where $u_{j}, v_{j}$ are holomorphic functions starting with terms of order at least two. One says that $\lambda=\left(\lambda_{1}, \ldots, \lambda_{n}\right)$ is non-resonant, if

$$
\lambda_{1}^{\alpha_{1}} \lambda_{2}^{\alpha_{2}} \cdots \lambda_{n}^{\alpha_{n}} \neq 1
$$

for all integers $\alpha_{1}, \ldots, \alpha_{n}$ with $\left|\alpha_{1}\right|+\cdots+\left|\alpha_{n}\right|>0$. G.D. Birkhoff [4] showed that under formal symplectic transformations, $\varphi$ is equivalent to the formal symplectic mapping

$$
\xi_{j} \rightarrow \lambda_{j} \xi_{j} e^{\omega_{j}}, \quad \eta_{j} \rightarrow \lambda_{j}^{-1} \eta_{j} e^{-\omega_{j}}, \quad \omega_{j}=\partial_{\zeta_{j}} H
$$

Supported in part by NSF grants DMS-9704835 and DMS-0096047.

Keywords: Levi-flat set - Segre variety - Holomorphic symplectic map - Birkhoff normal form.

Math. classification: 37G05 - 32V40 - 70H06. 
with $H$ a formal power series in the products $\zeta_{1}=\xi_{1} \eta_{1}, \ldots, \zeta_{n}=\xi_{n} \eta_{n}$ without constant term.

Analogous to work of C.L. Siegel [17] on Hamiltonian systems, a result of H. Rüssmann [12] says that as a rule the Birkhoff normal form (1.3) of $\varphi$ is not realizable by convergent symplectic transformations. In fact, one aspect of divergence for Birkhoff's normalization for Hamiltonian systems was understood by Siegel [16] much earlier. Recall that holomorphic functions invariant under a holomorphic symplectic mapping or the flow of a holomorphic Hamiltonian system are called first-integrals. Siegel [16] showed that there are Hamiltonian systems of degree of freedom larger than one that admit no first-integrals other than functions of their Hamiltonians. On the other hand, when admitting a certain system of first-integrals, a holomorphic symplectic mapping or Hamiltonian system can be transformed into the Birkhoff normal form by convergent transformations. This is the so-called integrability of Hamiltonian systems (or symplectic mappings) via first-integrals, which was already observed by Birkhoff [3] for the case of one degree of freedom. In general cases, such integrability results were obtained by Rüssmann [13] for analytic Hamiltonian systems with two degrees of freedom and by J. Vey [18] for analytic Hamiltonian systems and symplectic mappings of any degrees of freedom, for which the quadratic forms of the first-integrals satisfy a certain non-degeneracy condition. Most recently H. Ito [10] studied the convergence of Birkhoff's normalization for analytic Hamiltonian systems and symplectic mappings which admit certain first-integrals with degenerate quadratic forms. The reader is also referred to results of L.H. Eliasson [9] on smooth real Hamiltonian systems. We should mention that the results in [13], [18], and [10], based on the first-integral method, hold for both real and complex cases.

In case the holomorphic symplectic map is in its normal form (1.3), it is clear that for each $j$ the quantity $\xi_{j} \eta_{j}$ is invariant under the map. In particular the set defined by $\Re \xi_{j} \eta_{j}=0(j=1, \ldots, n)$ is invariant. More generally, if $H_{j}$ are holomorphic functions of $n$ variables, the set defined by $\Re\left\{H_{j}(\xi \eta)\right\}=0$ is invariant.

In Vey's theorem and in Ito's one has of course the existence of a (singular) foliation of $\mathbb{C}^{2 n}$ by ( $n$-codimensional) invariant sets. In the present work, we show the convergence of (the reduction to) the normal form under the sole hypothesis of just one appropriate invariant real analytic set containing the origin. The invariant set that we assume to exist is of co-dimension $n$ in $\mathbb{C}^{2 n}$, and Levi-flat, as above. A generic $n$-dimensional 
real analytic set in $\mathbb{C}^{2 n}$ is said to be Levi-flat if its regular set (away from singular points and $C R$ singularities) is foliated by $n$-dimensional complex manifolds. The main result of this paper is the convergence of the normalization under the assumption of the existence of an invariant set in two special cases (see below): (1.4) and (1.5).

We now state the following.

Theorem 1.1. - Let $\varphi$ be a holomorphic symplectic mapping of $\mathbb{C}^{2 n}$ given by (1.1)-(1.2), and let $M \subset \mathbb{C}^{2 n}$ be the real analytic set

$$
\Re\left\{\sum a_{j k} \xi_{k} \eta_{k}\right\}+R_{j}(\xi, \eta, \bar{\xi}, \bar{\eta})=0, \quad j=1, \ldots, n,
$$

where $\operatorname{rank}\left(a_{j k}\right)=n$ and $R_{j}(\xi, \eta, \bar{\xi}, \bar{\eta})=O(3)$ are real-valued analytic functions. Assume that $M$ is Levi-flat and $\varphi(M)=M$. Then $\varphi$ can be transformed into the Birkhoff normal form (1.3) by convergent symplectic transformations.

It turns out that the integrability of $\varphi$ in terms of Levi-flat invariant sets of the form (1.4) is well connected to its first-integrals; namely, we shall prove that $\varphi$ has $n$ first-integrals if it has a Levi-flat invariant set (1.4). Thus Theorem 1.1 follows eventually from the above-mentioned result of Vey. Our next result is the following.

Theorem 1.2. - Let $\varphi, M$ be as in Theorem 1.1 except that $M$ is given by

$$
\left|\xi_{j}\right|^{2}-\left|\eta_{\sigma(j)}\right|^{2}+R_{j}(\xi, \eta, \bar{\xi}, \bar{\eta})=0, \quad j=1, \ldots, n,
$$

where $\sigma$ is a permutation of $1, \ldots, n$ and $R_{j}(\xi, \eta, \bar{\xi}, \bar{\eta})=O(3)$ are realvalued analytic functions. Assume that $\varphi(M)=M$. Then $\left|\lambda_{\sigma(j)}\right| \cdot\left|\lambda_{j}\right|=1$ for $j=1, \ldots, n$. Moreover, $\varphi$ has $n$ formal first-integrals $\delta_{j} \kappa$ such that $\varphi$ can be transformed into (1.3) by convergent symplectic transformations, provided $\sigma^{2}=\mathrm{Id}$ and all $\delta_{j} \kappa \equiv 1$.

For the definition of $\delta_{j} \kappa\left(\delta_{j} \kappa \equiv 1\right.$ if $\left.\sigma=\mathrm{Id}\right)$, see (4.37) below.

In contrary to case (1.4), the Levi-flat invariant set (1.5), however, leads to meromorphic eigenfunctions of the holomorphic symplectic mappings. We shall show that such a mapping $\varphi$ has $n$ meromorphic functions $m_{j}$ satisfying $m_{j} \circ \varphi=\lambda_{j} \lambda_{\sigma(j)} m_{j}$. An example (end of Section 2) shows that it is not enough to assume the existence of an invariant Levi-flat $n$ codimensional set in $\mathbb{C}^{2 n}$ for $n \geq 2$, but in case of (1.5) we have no counterexample if one drops the restrictive hypotheses on the permutation $\sigma$ 
and on $\delta_{j} \kappa$. It also remains open if the Birkhoff normalization converges for the holomorphic symplectic mappings of $\mathbb{C}^{2}$ that have a Levi-flat real hypersurface. Only for a special case shall we prove the following.

THEOREM 1.3. - Let $\varphi$ be a holomorphic symplectic mapping of $\mathbb{C}^{2}$ given by (1.1)-(1.2). Let $M \subset \mathbb{C}^{2}: r=0$ be a real analytic Levi-flat hypersurface invariant under $\varphi$. Assume that the quadratic form of $r$ defines a real hypersurface in $\mathbb{C}^{2}$. Then $\varphi$ can be transformed into its Birkhoff normal form by convergent symplectic transformations.

Note that Theorem 3 is meant for the case $|\lambda|=1$, since Moser [11] already showed that a holomorphic symplectic mapping of $\mathbb{C}^{2}$ is always normalizable by convergent transformations if $|\lambda| \neq 1$.

The present paper relies on some techniques used in very recent joint work of D. Burns and the author [6], where singular Levi-flat real analytic hypersurfaces of $\mathbb{C}^{n}$ are studied. These techniques will allow us to construct holomorphic first-integrals or meromorphic eigenfunctions for the holomorphic symplectic mappings. Motivated by applications to holomorphic symplectic mappings, we shall also extend some results in [6] to Levi-flat sets of higher codimensions. One of main ingredients used in [6] is that of Segre varieties, which is a family of invariant complex varieties associated to a real analytic hypersurface in complex space [15]. In dealing with singular Levi-flat structure one encounters with difficulties of constructing formal normal forms. In [6] and in this work, the Segre varieties serve as an essential tool to avoid the formal normal forms of Levi-flat sets. As another application of Segre varieties we shall also use Segre varieties to prove part of Theorem 1.3 directly (see Proposition 4.2). The reader is also referred to work of E. Bedford [2] on the domain of holomorphy of complements of singular Levi-flat hypersurfaces. For other applications of Segre varieties, see work of S.M. Webster [19] on algebraic real hypersurfaces with non-degenerate Levi-form and work of DiederichFornaess [8] on complex varieties in real analytic sets.

The paper is organized as follows. Sections 2 and 3 consist in a preliminary study of Levi-flat invariant sets of codimension $n$. In Section 2, we study those Levi-flat sets defined by real quadratic forms. Their classification happens to be already non-trivial, and in fact we succeed only in classifying Levi-flat sets of special types. In Section 3, the Levi-flat sets that have been studied serve as models for more general Levi-flat sets. At the expense of having the hypotheses looking more artificial, the reader can 
read Section 3 without having read Section 2. Also, the results but not the proofs in Section 3 are to be used in Section 4.

In Section 4, the simplified equations that we found for the Leviflat invariant sets allow one to study the defining functions of those sets, i.e., functions whose at least the common zero level set is invariant. From this study one is able to get either enough first-integrals, or meromorphic eigenfunctions.

In case enough first-integrals are obtained, the convergence of normal forms follows from Vey's theorem. This however does not establish Theorem 1.2, which correspond to a case for which in Section 4 one obtains only meromorphic eigenfunctions. This latter case is treated in Section 5, using a KAM method.

In Section 6 we shall formulate analogous results for holomorphic Hamiltonian systems, based on an observation regarding normal forms of the time-one mappings of Hamiltonian systems. The paper is concluded with an appendix about two equivalent Birkhoff normal forms of holomorphic symplectic mappings.

Acknowledgment. The author is indebted to Dan Burns for the insights in Segre varieties through the collaboration [6]. The author would also like to thank Jean-Pierre Rosay for helpful suggestions.

\section{Invariant Levi-flat sets defined by real quadratic forms.}

In this section we shall study certain classes of Levi-flat sets of high codimension in $\mathbb{C}^{2 n}$ defined by real quadratic forms. Here the classification is not complete. In fact, we shall only consider 4 families of Levi-flat sets of high codimension under an additional condition that the sets are invariant under an elliptic complex linear symplectic transformation. A complete classification of Levi-flat quadratic hypersurfaces of $\mathbb{C}^{n}$ is in [6].

Recall that a germ $M$ of real analytic set of dimension $k$ at $0 \in \mathbb{C}^{n}$ admits a decomposition $M=M^{*} \cup M_{s}$, where $M^{*}$ consists of points $x$ near which $M$ is a $k$-dimensional submanifold of $\mathbb{C}^{n}$. By a result of Bruhat and Cartan [5], if $M$ is irreducible, $M^{*}$ is the union of a finite number of topological components $M_{j}^{*}$ with the origin of $\mathbb{C}^{n}$ in the closure of each $M_{j}^{*}$. We define the $C R$ dimension of $M$ to be

$$
\liminf _{M^{*} \ni p \rightarrow 0} \operatorname{dim}_{\mathbb{C}} T_{p} M \cap \sqrt{-1} T_{p} M \equiv \operatorname{dim}_{C R} M .
$$


Let $M_{c s}$ be the set of points $x \in M^{*}$ at which the complex dimension of $T_{x} M \cap \sqrt{-1} T_{x} M$ is larger than $\operatorname{dim}_{C R} M$. Then $M_{c s}$ is a real analytic subset of $M^{*}$. We say that $M$ is Levi-flat, if $M_{c} \equiv M^{*} \backslash M_{c s}$ is foliated by complex submanifolds of dimension $\operatorname{dim}_{C R} M$. Notice that we allow $M_{c s}$ to contain some components of $M^{*}$. In general, one has $\operatorname{dim}_{C R} M \geq$ $\max \{\operatorname{dim} M-n, 0\}$. We say that $M$ is generic, or of minimal $C R$ dimension, when $\operatorname{dim}_{C R} M=\max \{\operatorname{dim} M-n, 0\}$, i.e., when $T_{x} M_{c}+\sqrt{-1} T_{x} M_{c}=\mathbb{C}^{n}$ for all $x \in M_{c}$, or $T_{x} M_{c} \cap \sqrt{-1} T_{x} M_{c}=0$ for all $x \in M_{c}$. In the latter case $M_{c}$ is said to be totally real. We shall also use a standard fact that the real analytic submanifold $M_{c}$ is Levi-flat, if and only if the Lie brackets $\left[L_{i}, \bar{L}_{j}\right]$ remain in the span of $L_{1}, \ldots, L_{m}, \bar{L}_{1}, \ldots, \bar{L}_{m}$ for any local basis $L_{1}, \ldots, L_{m}\left(m=\operatorname{dim}_{C R} M\right)$ of $(1,0)$ tangent vectors of $M_{c}$, i.e., tangent vectors of $M_{c}$ of the form $\sum_{j=1}^{n} a_{j}(z, \bar{z}) \frac{\partial}{\partial z_{j}}$ with $a_{j}$ being analytic.

We shall need some basic properties about the complexification of real analytic varieties. Recall that a germ $M$ of real analytic variety of $\mathbb{R}^{n}$ at 0 is contained in a unique germ $M^{c}$ of complex variety at $0 \in \mathbb{C}^{n}$ such that $M=M^{c} \cap \mathbb{R}^{n}$, and such that germs of holomorphic function at $0 \in \mathbb{C}^{n}$ vanishing on $M^{c}$ are precisely the germs of complex-valued real analytic function at $0 \in \mathbb{R}^{n}$ that vanish on $M$. We shall refer the germ $M^{c}$ as the Bruhat-Whitney-Cartan complexification of $M$. A result of H. Cartan [7] says that $M$ is irreducible if and only if $M^{c}$ is irreducible; consequently, a germ of real analytic function vanishing on an open subset of $M^{*}$, of which the closure contains the origin, vanishes entirely on the germ $M$.

The main purpose of this section is to study Levi-flat sets that are defined by quadratic forms and invariant under the $\mathbb{C}$-linear symplectic mapping

$$
\Lambda: \xi_{j} \rightarrow \lambda_{j} \xi_{j}, \quad \eta_{j} \rightarrow \lambda_{j}^{-1} \eta_{j}, \quad j=1, \ldots, n,
$$

where $\lambda_{1}, \ldots, \lambda_{n}$ satisfy the non-resonance condition (1.2). Throughout the section, we assume that $\Lambda$ is elliptic, that is that $\left|\lambda_{j}\right|=1, j=1, \ldots, n$.

A complex-valued quadratic form on $\mathbb{C}^{2 n}$ is a quadratic homogeneous polynomial in $\xi, \eta, \bar{\xi}, \bar{\eta}$; the space of all complex-valued quadratic forms will be denoted by $E$. Let $E^{p s h}$ be the set of complex-valued quadratic forms containing no terms of the form $\xi^{\alpha} \eta^{\beta} \bar{\xi}^{\gamma} \bar{\eta}^{\delta}$ with $|\alpha|+|\beta|=1$, and $E^{h r m}$ the set of quadratic forms containing only the terms $\xi^{\alpha} \eta^{\beta} \bar{\xi}^{\gamma} \bar{\eta}^{\delta}$ with $|\alpha|+|\beta|=1$. Sending $f$ to $f \circ \Lambda$, the transformation $\Lambda: E \rightarrow E$ is $\mathbb{C}$-linear. Put

$$
\lambda_{-j}=\bar{\lambda}_{j}, \quad \xi_{-j}=\bar{\xi}_{j}, \quad \eta_{-j}=\bar{\eta}_{j}
$$


Notice that (2.1) holds for $j= \pm 1, \ldots, \pm n$. The eigenvalues of the linear transformation $\Lambda: E \rightarrow E$ are 1 and $\lambda_{i} \lambda_{j}(i+j \neq 0)$, to which the corresponding eigen-spaces are

$$
\begin{aligned}
E_{1} & =\bigoplus_{i=1}^{n} \mathbb{C} \cdot \xi_{i} \eta_{i} \oplus \mathbb{C} \cdot \bar{\xi}_{i} \bar{\eta}_{i} \oplus \mathbb{C} \cdot \xi_{i} \bar{\xi}_{i} \oplus \mathbb{C} \cdot \eta_{i} \bar{\eta}_{i}, \\
E_{i j} & =\mathbb{C} \cdot \xi_{i} \xi_{j} \oplus \mathbb{C} \cdot \bar{\eta}_{i} \bar{\eta}_{j} \oplus \mathbb{C} \cdot \xi_{i} \bar{\eta}_{j} \oplus \mathbb{C} \cdot \bar{\eta}_{i} \xi_{j}
\end{aligned}
$$

with

$$
i, j= \pm 1, \ldots, \pm n, \quad j \geq i, \quad i+j \neq 0 .
$$

Let $\mathcal{Q}$ be the vanishing set of finitely many real quadratic forms on $\mathbb{C}^{2 n}$, and let $\mathcal{I}=\mathcal{I}_{\mathcal{Q}}$ be the set of complex-valued quadratic forms vanishing on $\mathcal{Q}$. Assume that $\mathcal{Q}$ is invariant under $\Lambda$, so $\mathcal{I}$ is invariant under $\Lambda$ also. ¿From the theory of linear algebra one knows that

$$
\mathcal{I}=\mathcal{I} \cap E_{1} \oplus \bigoplus \mathcal{I} \cap E_{i j} \equiv \mathcal{I} \cap E_{1} \oplus \mathcal{I} \cap E_{0} .
$$

We shall only classify the Levi-flat sets $\mathcal{Q}$ for which $\mathcal{I}$ is contained in one of the subspaces

$$
E_{1} \cap E^{p s h}, \quad E_{1} \cap E^{h r m}, \quad E_{0} \cap E^{p s h}, \quad E_{0} \cap E^{h r m} .
$$

We start with the following.

Proposition 2.1. - Let $\mathcal{Q} \subset \mathbb{C}^{2 n}$ be a generic real analytic set defined by real quadratic forms. Assume that $\mathcal{Q}$ has codimension $m \leq 2 n$ and $\mathcal{I}_{\mathcal{Q}}$ is contained in $E^{p s h} \cap E_{1}$. Then there exists an $m \times n$ matrix $\left(a_{i j}\right)$ of rank $m$ such that

$$
\mathcal{Q}: \Re\left\{\sum a_{i j} \xi_{j} \eta_{j}\right\}=0, \quad i=1, \ldots, m .
$$

Conversely, (2.2) is a Levi-flat real analytic set in $\mathbb{C}^{2 n}$ of minimal $C R$ dimension $2 n-m$, provided the rank of $\left(a_{i j}\right)$ is $m$.

Proof. - Take $k$ quadratic forms $q_{i}(\xi \eta)=\sum_{j=1}^{n} a_{i j} \xi_{j} \eta_{j}$ so that $\Re q_{1}, \ldots, \Re q_{k}$ form a basis for real quadratic forms vanishing on $\mathcal{Q}$. Since $\mathcal{Q}$ is generic and of codimension at most $2 n$, then $\mathcal{Q}$ is not contained in any proper complex subvariety of $\mathbb{C}^{2 n}$. This implies that $q_{1}, \ldots, q_{k}$ are linearly independent over $\mathbb{C}$. For the proof, we assume for the sake of contradiction that $q_{k}=c_{1} q_{1}+\cdots+c_{k} q_{k-1}$. Then

$$
\sum_{j=1}^{k-1} \Im c_{j} \cdot \Im q_{j}=-\Re q_{k}+\sum_{j=1}^{k-1} \Re c_{j} \Re q_{j}
$$

TOME 51 (2001), FASCICULE 1 
vanishes on $\mathcal{Q}$. Hence, $\sum_{j=1}^{k-1} \Im c_{j} \cdot q_{j}$ vanishes on $\mathcal{Q}$. Since $\Re q_{1}, \ldots, \Re q_{k}$ are linearly independent over $\mathbb{R}$, then at least one of $\Im c_{j}$ is nonzero. Therefore, $\mathcal{Q}$ is contained in the complex variety $\sum_{j=1}^{k-1} \Im c_{j} \cdot q_{j}=0$, which is a contradiction.

To show that $k=m$, we need only to verify the last statement in the proposition. Without loss of generality, one may assume that $\left(a_{i j}\right)_{1 \leq i, j \leq m}$ is nonsingular. Then $\mathcal{Q} \backslash \cup_{j=1}^{m}\left\{\xi_{j}=0\right\}$ is parameterized by

$$
\eta_{i}=\sqrt{-1}\left\{\sum_{j \leq m} b_{i j} t_{j}+\sum_{j>m} c_{i j} \xi_{j} \eta_{j}\right\} / \xi_{i}, \quad i=1, \ldots, m
$$

with $\left(b_{i j}\right)=\left(a_{i j}\right)_{1 \leq i, j \leq m}^{-1}$ and $t_{i} \in \mathbb{R}$. This shows that $\mathcal{Q} \backslash \cup_{j=1}^{m}\left\{\xi_{j}=0\right\}$ is a generic Levi-flat $C R$ manifold of $C R$ dimension $2 n-m$. Next, we want to show that $\mathcal{Q} \cap \cup_{j=1}^{m}\left\{\xi_{j}=0\right\}$ has dimension less than $4 n-m$. It suffices to verify that $\mathcal{Q}^{\prime}=\mathcal{Q} \cap\left\{\xi_{1}=0\right\}$ has dimension less than $4 n-m$. Note that the rank of $\left(a_{i j}\right)_{1 \leq i \leq m, 2 \leq j \leq m}$ is $m-1$. Without loss of generality, we may further assume that $\left(a_{i j}\right)_{2 \leq i, j \leq m}$ has rank $m-1$. Obviously, $\mathcal{Q}^{\prime}$ is contained in $\mathbb{C} \times \mathcal{Q}^{\prime \prime}$ for $\mathcal{Q}^{\prime \prime} \subset \mathbb{C}^{2 n-2}: \Re\left\{\sum_{j=2}^{m} a_{i j} \xi_{j} \eta_{j}\right\}=0, i=2, \ldots, m$. Applying induction on $n$ and $m$, one can verify that $\mathcal{Q} \cap\left\{\xi_{1}=\eta_{1}=0\right\}$ is a real analytic set of dimension at most $4(n-1)-(m-1)$; hence $\operatorname{dim} \mathcal{Q}^{\prime}<4 n-m$. The proof of the proposition is complete.

Proposition 2.2. - Let $\mathcal{Q} \subset \mathbb{C}^{2 n}$ be an irreducible generic real analytic set defined by real quadratic forms and of dimension at least $2 n$. Assume that $\Lambda(\mathcal{Q})=\mathcal{Q}$, and that $\mathcal{I}$ is contained in $E^{p s h} \cap E_{0}$. There is a $\mathbb{C}$ linear symplectic transformation which leaves $\Lambda$ in the diagonal form (2.1) and transforms $\mathcal{Q}$ into the set

$$
\xi_{j} \eta_{\sigma(j)}+\mu_{j} \bar{\xi}_{\sigma(j)} \bar{\eta}_{j}=0, \quad j=1, \ldots, 2 L
$$

with $\sigma(2 j-1)=2 j=\sigma^{-1}(2 j-1)$ for $j=1, \ldots, L$, and

$$
\left|\mu_{j}\right|=1, \quad \mu_{\sigma(j)}=\mu_{j}
$$

Proof. - Since $\mathcal{I}$ is invariant under $\Lambda$, we have $\mathcal{I}=\oplus I_{i j}^{p s h}$ for

$$
\mathcal{I}_{i j}^{p s h} \equiv \mathcal{I} \cap E^{p s h} \cap E_{i j} .
$$

Assume that $\mathcal{I}_{i j}^{p s h} \neq\{0\}$. We first want to show that $\operatorname{dim}_{\mathbb{C}} \mathcal{I}_{i j}^{\text {psh }}=1$. Without loss of generality, one may assume that $i, j>0$. Assume for the sake of contradiction that $\operatorname{dim}_{\mathbb{C}} \mathcal{I}_{i j}^{p s h}>1$. Since $\operatorname{dim}_{\mathbb{C}} E_{i j}^{p s h}=2$ then 
$\mathcal{I}_{i j}^{p s h}=E_{i j}^{p s h} ;$ hence, $\mathcal{Q}$ is contained in $\xi_{i} \xi_{j}=0$, which contradicts that $\mathcal{Q}$ is generic and of dimension at least $2 n$. Next, we want to show that

$$
\operatorname{dim}_{\mathbb{C}} \mathcal{I}_{i j}^{p s h}+\operatorname{dim}_{\mathbb{C}} \mathcal{I}_{i^{\prime} j^{\prime}}^{p s h} \leq 1
$$

for $\{i, j\} \neq\left\{i^{\prime}, j^{\prime}\right\}$ and $\{i, j\} \cap\left\{i^{\prime}, j^{\prime}\right\} \neq \emptyset$. Let us focus on the case $i, j>0$, and other cases can be checked similarly. Assume for the sake of contradiction that on $\mathcal{Q}$

$$
a \xi_{i} \xi_{j}+b \bar{\eta}_{i} \bar{\eta}_{j}=0, \quad c \xi_{i} \xi_{j^{\prime}}+d \bar{\eta}_{i} \bar{\eta}_{j^{\prime}}=0
$$

for some $a, b, c, d \neq 0$. Eliminating $\xi_{i}$ and $\bar{\eta}_{i}$ yields

$$
a d \xi_{j} \bar{\eta}_{j^{\prime}}-b c \xi_{j^{\prime}} \bar{\eta}_{j}=0
$$

on $\mathcal{Q} \backslash\left(\left\{\xi_{i}=0\right\} \cup\left\{\xi_{j^{\prime}}=0\right\}\right)$. Since $\mathcal{Q}$ is irreducible and generic, then (2.6) holds on $\mathcal{Q}$ also, which contradicts that $\mathcal{I} \cap E^{h r m}=\{0\}$.

Next, we want to show that all $I_{i i}^{p s h}=0$. Otherwise, one may assume that $I_{1,1}^{p s h} \neq 0$, so $\mathcal{Q}$ is defined by the vanishing of $\xi_{1}^{2}-a \bar{\eta}_{1}^{2}$ and other quadratic forms independent of $\xi_{1}, \eta_{1}$. Denote by $\mathcal{Q}^{\prime} \subset \mathbb{C}^{2 n-2}$ the vanishing set of the complex-valued quadratic forms in $\mathcal{I}$ that are independent of $\xi_{1}, \eta_{1}$. Obviously, the decomposition

$$
\mathcal{Q}=\left(\left\{\xi_{1}+\sqrt{a} \bar{\eta}_{1}=0\right\} \cup\left\{\xi_{1}-\sqrt{a} \bar{\eta}_{1}=0\right\}\right) \times \mathcal{Q}^{\prime}
$$

contradicts that $\mathcal{Q}$ is irreducible.

To achieve (2.3), let $\mathcal{I}$ contain one of quadrics

$$
\xi_{i} \eta_{j}+a \bar{\eta}_{i} \bar{\xi}_{j}, \quad \xi_{i} \xi_{j}+a \bar{\eta}_{i} \bar{\eta}_{j}, \quad \eta_{i} \eta_{j}+a \bar{\xi}_{i} \bar{\xi}_{j}
$$

with $i \neq j$ and $a \neq 0$. By substituting $\left(\eta_{j},-\xi_{j}\right)$ for $\left(\xi_{j}, \eta_{j}\right)$ in the second quadratic form and replacing the third quadratic form by its conjugate divided by $\bar{a}$, one may assume that the first quadratic form is in $\mathcal{I}$. Next, interchange $\left(\xi_{i}, \eta_{i}\right)$ with $\left(\xi_{1}, \eta_{1}\right)$ and $\left(\xi_{j}, \eta_{j}\right)$ with $\left(\xi_{2}, \eta_{2}\right)$, respectively. Thus $\mathcal{I}$ contains

$$
\xi_{1} \eta_{2}+\mu_{1} \bar{\eta}_{1} \bar{\xi}_{2}, \quad \mu_{1} \neq 0 .
$$

By the transformation $\left(\xi_{1}, \eta_{1}\right) \rightarrow\left(\sqrt{\left|\mu_{1}\right|} \xi_{1}, \eta_{1} / \sqrt{\left|\mu_{1}\right|}\right)$, one gets $\left|\mu_{1}\right|=1$. Inductively, one finds the remaining coordinates $\left(\xi_{3}, \eta_{3}, \ldots, \xi_{n}, \eta_{n}\right)$ so that $\mathcal{Q}$ is given by (2.3)-(2.4). The proof of the proposition is complete.

Analogous to Proposition 2.2, we have the following.

Proposition 2.3. - Let $\mathcal{Q} \subset \mathbb{C}^{2 n}$ be an irreducible generic real analytic set defined by real quadratic forms and of dimension at least $2 n$. 
Assume that $\mathcal{Q}$ is invariant under $\Lambda$, and that $\mathcal{I}$ is contained in $E^{h r m} \cap E_{0}$ and satisfies the non-degeneracy conditions

$$
\operatorname{dim}_{C} \mathcal{I}_{i j}^{h r m} \cdot \operatorname{dim}_{C} \mathcal{I}_{j k}^{h r m}=0
$$

for all distinct $i, j, k$. Then there is a $\mathbb{C}$-linear symplectic transformation which leaves $\Lambda$ in the diagonal form (2.1) and transforms $\mathcal{Q}$ into

$$
\xi_{j} \bar{\xi}_{\sigma(j)}-\mu_{j} \bar{\eta}_{j} \eta_{\sigma(j)}=0, \quad j=1, \ldots, 2 L
$$

with $\mu_{\sigma(j)}=\bar{\mu}_{j},\left|\mu_{j}\right|=1$, and $\sigma(2 j-1)=2 j=\sigma^{-1}(2 j-1)$ for $j=1, \ldots, L$.

The proof is almost the same as that of Proposition 2.2, except (2.7), replacing (2.5), is part of the assumptions of the proposition. One can show that $\mathcal{Q}$ is equivalent to $\xi_{j} \bar{\eta}_{\sigma(j)}+\mu_{j} \bar{\eta}_{j} \xi_{\sigma(j)}=0, j=1, \ldots, 2 L$. However, the latter is equivalent to (2.8) by a symplectic transformation. The details are left to the reader.

We depart from the symplectic coordinates for a moment.

Proposition 2.4. - Let $\mathcal{Q} \subset \mathbb{C}^{n}$ be defined by the vanishing of some linear combinations of $z_{1} \bar{z}_{1}, \ldots, z_{n} \bar{z}_{n}$. Assume that $\mathcal{Q}$ is generic, Leviflat, and of codimension $m \leq n$. Then $m$ is less than $n$ and rearranging $z_{1}, \ldots, z_{n}$ gives

$$
\mathcal{Q}: z_{j} \bar{z}_{j}-c_{j} z_{k_{j}} \bar{z}_{k_{j}}=0, \quad j=1, \ldots, m
$$

for some $c_{j}>0$ and $k_{j}>m$.

Proof. - For the space of $\mathbb{R}$-linear combinations of $z_{1} \bar{z}_{1}, \ldots, z_{n} \bar{z}_{n}$ that vanish on $\mathcal{Q}$, choose a basis $q_{1}, \ldots, q_{k}$ so that for a possible rearrangement of $z_{1}, \ldots, z_{n}$

$$
q_{j}(z, \bar{z})=z_{j} \bar{z}_{j}-\sum_{\alpha>k} a_{j \alpha} z_{\alpha} \bar{z}_{\alpha}, \quad j=1, \ldots, k .
$$

Since $\mathcal{Q}$ is not contained in $\cup_{j=1}^{n} z_{j}=0$, then

$$
D=\left\{\left(z_{k+1}, \ldots, z_{n}\right) \mid \sum_{l>k} a_{j l} z_{l} \bar{z}_{l}>0, j=1, \ldots, k\right\}
$$

is a nonempty open set in $\mathbb{C}^{n-k}$, of which the boundary contains the origin. Obviously, $\left\{q_{1}=\cdots=q_{k}=0\right\} \backslash \cup_{j=1}^{k}\left\{z_{j}=0\right\}$ is a smooth submanifold in $\mathbb{C}^{n}$ of codimension $k$; in particular, $k \geq m$. On the other hand, if $k>m$, then $\mathcal{Q}^{*}$ is contained in $\cup_{j=1}^{k}\left\{z_{j}=0\right\}$, which contradicts that $\mathcal{Q}$ is generic. Therefore, $k=m$. 
To determine $a_{j \alpha}$ we consider $(1,0)$ tangent vectors of $\mathcal{Q}_{c} \backslash \cup_{j=1}^{k}\left\{z_{j}=\right.$ $0\}$, spanned by

$$
L_{\alpha}=\sum_{j=1}^{m} a_{j \alpha} \frac{\bar{z}_{\alpha}}{\bar{z}_{j}} \frac{\partial}{\partial z_{j}}+\frac{\partial}{\partial z_{\alpha}}, \quad \alpha>m
$$

One has

$$
\left[L_{\alpha}, \bar{L}_{\beta}\right]=\sum_{j=1}^{m} a_{j \alpha} a_{j \beta} \frac{z_{\alpha} \bar{z}_{\beta}}{z_{j} \bar{z}_{j}^{2}} \frac{\partial}{\partial \bar{z}_{j}}-\sum_{j=1}^{m} a_{j \alpha} a_{j \beta} \frac{z_{\alpha} \bar{z}_{\beta}}{\bar{z}_{j} z_{j}^{2}} \frac{\partial}{\partial z_{j}}, \quad \alpha \neq \beta .
$$

Since $\mathcal{Q}$ is Levi-flat, the Lie bracket $\left[L_{\alpha}, \bar{L}_{\beta}\right]$, restricted to $\mathcal{Q}_{c} \backslash \cup\left\{z_{j}=0\right\}$, is locally in the span of vector fields $(2.11)$ and their conjugates. This implies that $\left[L_{\alpha}, \bar{L}_{\beta}\right]$ vanishes on $\mathcal{Q}_{c} \backslash \cup\left\{z_{j}=0\right\}$, i.e.,

$$
a_{j \alpha} a_{j \beta} \frac{z_{\alpha} \bar{z}_{\beta}}{z_{j} \bar{z}_{j}^{2}}=0, \quad \text { on } \mathcal{Q} \backslash \cup_{j=1}^{m}\left\{z_{j}=0\right\} .
$$

Since $\mathcal{Q} \backslash \cup_{j=1}^{m}\left\{z_{j}=0\right\}$ is not contained in $z_{\alpha} z_{\beta}=0$, then $a_{j \alpha} a_{j \beta}=0$ for $\alpha \neq \beta$. On the other hand, from (2.10) one knows that at least one of $a_{j \alpha}(\alpha>m)$ is positive. This shows that $\mathcal{Q}$ has the form (2.9). The proof of the proposition is complete.

Returning to symplectic coordinates, we now want to prove the following.

Proposition 2.5. - Let $\mathcal{Q} \subset \mathbb{C}^{2 n}$ be a Levi-flat real analytic set defined by elements in $E^{h r m} \cap E_{1}$. Assume that $\mathcal{Q}$ is generic and of codimension $m \leq 2 n$, and that $\mathcal{Q}$ contains no $\mathbb{C}$-linear coordinate subspaces (i.e., vanishing sets of some of $\xi_{1}, \eta_{1}, \ldots, \xi_{n}, \eta_{n}$ ) of dimension greater than $2 n-2 m$. Then under a change of symplectic coordinates which leaves $\Lambda$ in the form (2.1), $\mathcal{Q}$ is given by

$$
\left|\xi_{j}\right|^{2}-\mu_{j}\left|\eta_{\sigma(j)}\right|^{2}=0, \quad j=1, \ldots, m,
$$

where $\sigma:\{1, \ldots, m\} \rightarrow\{1, \ldots, n\}$ is injective, and $\mu_{j}$ satisfy

$$
\mu_{j}= \begin{cases}1 / \mu_{\sigma(j)}>0 & \text { if } \tau_{j} \text { is even and positive, } \\ 1 & \text { otherwise }\end{cases}
$$

with $\tau_{j}$ being the smallest positive integer satisfying $\sigma^{\tau_{j}}(j)=j\left(\tau_{j}=0\right.$ when $\sigma^{k}(j)>m$ for some $\left.k\right)$.

Proof. - In view of Proposition 2.4, such a set takes the form (2.9) with $z_{1}, \ldots, z_{2 n}$ being a permutation of $\xi_{1}, \eta_{1}, \ldots, \xi_{n}, \eta_{n}$. Since $\mathcal{Q}$ does not contain any $\mathbb{C}$-linear coordinate subspace of dimension greater than 
$2 n-2 m$, all $k_{1}, \ldots, k_{m}$ in $(2.9)$ are distinct; consequently, $m \leq n$ and $\mathcal{Q}$ is given by $m$ equations

$$
\left|\xi_{i_{1}}\right|^{2}=c_{i_{1}}^{\prime}\left|\xi_{\alpha\left(i_{1}\right)}\right|^{2}, \quad\left|\xi_{i_{2}}\right|^{2}=c_{i_{2}}^{\prime \prime}\left|\eta_{\beta\left(i_{2}\right)}\right|^{2}, \quad\left|\eta_{i_{3}}\right|^{2}=c_{i_{3}}^{\prime \prime}\left|\eta_{\gamma\left(i_{3}\right)}\right|^{2},
$$

in which $c_{i_{1}}^{\prime}, c_{i_{2}}^{\prime \prime}, c_{i_{3}}^{\prime \prime \prime}$ are positive, and indices $i_{1}, i_{2}, i_{3}$ run over subsets $I_{1}, I_{2}, I_{3}$ of $\{1, \ldots, n\}$, respectively. Moreover, $I_{1}, I_{2}$ and $\alpha\left(I_{1}\right)$ are mutually disjoint, and so are $I_{3}, \beta\left(I_{2}\right)$, and $\gamma\left(I_{3}\right)$. We shall first permute the symplectic coordinates to eliminate the first and last groups of equations in (2.14). For each $j \in \alpha\left(I_{1}\right)$, we change the coordinates $\left(\xi_{j}, \eta_{j}\right)$ to $\left(-\eta_{j}, \xi_{j}\right)$, and for each $k \in \beta^{-1}\left(\alpha\left(I_{1}\right) \cap \beta\left(I_{2}\right)\right)$, we change the coordinates $\left(\xi_{k}, \eta_{k}\right)$ to $\left(-\eta_{k}, \xi_{k}\right)$ and replace $c_{k}^{\prime \prime}$ with $1 / c_{k}^{\prime \prime}$. Thus, equations in the first group are eliminated. Next, we apply the coordinate change $\left(\xi_{j}, \eta_{j}\right)$ to $\left(-\eta_{j}, \xi_{j}\right)$ for each $j \in I_{3}$ and $j \in \beta^{-1}\left(I_{3} \cap \beta\left(I_{2}\right)\right)$. After the above coordinate changes, only the second group of equations in (2.14) remains. By a permutation of symplectic coordinates, we achieve (2.12) for an injective $\sigma$.

To obtain the normalization (2.13), we apply induction on the number of equations in (2.12). If $m=1$, one readily obtains (2.13) by applying the transformation $\left(\xi_{j}, \eta_{j}\right) \rightarrow\left(\sqrt{\mu_{1}} \xi_{j}, \eta_{j} / \sqrt{\mu_{1}}\right)$ for $j=1, \sigma(1)$.

For induction, we assume that (2.13) can be achieved if the number of equations in (2.12) is less than $m$. We first consider the case there exists the smallest integer $\tau_{1}$ with $\sigma^{\tau_{1}}(1)=1$. Note that such $\tau_{1}$ always exists if $\sigma^{k}(1) \leq m$ for all integer $k$. In this case, the equations in (2.12) break into two groups: the equations involving only $\left(\xi_{\sigma^{k}(1)}, \eta_{\sigma^{k}(1)}\right)$ for $k=1, \ldots, \tau_{1}$ and the ones not involving any of those variables. If both groups are non-empty, (2.12) follows from the induction assumption. Thus, one may assume, without loss of generality, that $\tau_{1}=m, \sigma^{j}(1)=j+1$ for $j=1, \ldots, m-1$.

Consider a symplectic transformation

$$
\xi_{j} \rightarrow c_{j} \xi_{j}, \quad \eta_{j} \rightarrow \eta_{j} / c_{j}, \quad c_{j}>0, \quad j=1, \ldots, n .
$$

The transformation (2.15) sends (2.12) to $\left|\xi_{j}\right|^{2}-\tilde{\mu}_{j}\left|\eta_{\sigma(j)}\right|^{2}=0$ with $\tilde{\mu}_{j}=c_{j}^{2} c_{j+1}^{2} \mu_{j}$. Put $c_{1}=1$ and $c_{j}=1 /\left(c_{j-1} \sqrt{\mu_{j-1}}\right)$ for $j=2, \ldots, m$. Then $\tilde{\mu}_{j}=1$ for $j=1, \ldots, m-1$. To normalize $\tilde{\mu}_{m}$, consider first the case that $m$ is even. In this case

$$
\delta_{1} \mu \equiv \mu_{1} \mu_{2}^{-1} \mu_{3} \cdots \mu_{m}^{-1}
$$

is invariant under (2.15). Therefore, (2.12) are equivalent to $\left|\xi_{j}\right|^{2}-$ $\mu_{j}^{\prime}\left|\eta_{j+1}\right|^{2}=0, j=1, \ldots, m$ for $\mu_{1}^{\prime}=(\delta, \mu)^{1 / m}$ and $\mu_{\sigma(j)}^{\prime}=1 / \mu_{j}^{\prime}$, since $\delta_{1} \mu^{\prime}=\delta_{1} \mu$. Consider now the case that $m$ is odd. In this case we apply 
another transformation (2.15) with $c_{1}=\left(\mu_{m}^{\prime}\right)^{-1 / 4}$ and $c_{j+1}=1 / c_{j}$ for $j=1, \ldots, m-1$, which gives us $(2.12)$ with $\mu_{j}=1$ for $j=1, \ldots, m$.

We now consider the case that $\sigma^{k}(1)=m^{\prime}>m$ for some $k$. In this case $1 \leq \tau^{-1}\left(m^{\prime}\right) \leq m$. Let $\tau$ be the largest integer such that $\sigma^{-\tau}\left(m^{\prime}\right)$ is well-defined. The equations in (2.12) are divided into two groups: the ones involving $\eta_{m^{\prime}}, \xi_{\sigma^{-1}\left(m^{\prime}\right)}, \eta_{\sigma^{-1}\left(m^{\prime}\right)}, \ldots, \xi_{\sigma^{-\tau}\left(m^{\prime}\right)}$ only and the ones not involving $\xi_{\sigma^{-k}\left(m^{\prime}\right)}, \eta_{\sigma^{-k}\left(m^{\prime}\right)}$ for $k=0, \ldots, \tau$. Thus by the induction assumption, one may assume that $\tau=m-1$. Put $j_{0}=\sigma^{-m+1}\left(m^{\prime}\right)$ and $j_{k}=\sigma^{k}\left(j_{0}\right)$ for $k=1, \ldots, m-1$. Thus, (2.12) becomes

$$
\left|\xi_{k_{j}}\right|^{2}-\mu_{k_{j}}\left|\eta_{k_{j+1}}\right|^{2}=0, \quad j=0, \ldots, m-1
$$

with $j_{0}, \ldots, j_{m-1} \leq m$ being distinct and $j_{m}=m^{\prime}>m$. Take $c_{k_{m-1}}=1$, and $c_{k_{j}}=1 /\left(c_{k_{j+1}} \sqrt{\mu_{k_{j}}}\right)$ for $k=m-2, \ldots, 0$. Then applying the transformation $(2.15)$ one gets $\mu_{k_{j}}=1$ for $j=0, \ldots, m-1$. The proposition is proved by induction.

To conclude this section we remark that, for holomorphic symplectic mappings, having a Levi-flat invariant set of minimal $C R$ dimension is not a sufficient condition for the convergence of their normalizations. In fact, the sets $M_{2}, \ldots, M_{5}$ below are combinations of 4 types of Levi-flat sets (2.2), (2.3), (2.8) and (2.12).

Examples. - The following are Levi-flat sets of minimal $C R$ dimension:

$$
\begin{aligned}
& M_{1} \subset \mathbb{C}^{4}: \xi_{2}^{2}-\bar{\eta}_{2}^{2}=0, \\
& M_{2} \subset \mathbb{C}^{6}: \xi_{2} \eta_{2}+\overline{\xi_{2} \eta_{2}}=\xi_{3} \xi_{3}+\overline{\eta_{3} \eta_{3}}=0, \\
& M_{3} \subset \mathbb{C}^{6}:\left|\xi_{2}\right|^{2}-\left|\eta_{2}\right|^{2}=\xi_{2} \bar{\eta}_{3}+\xi_{3} \bar{\eta}_{2}=0, \\
& M_{4} \subset \mathbb{C}^{6}:\left|\xi_{2}\right|^{2}-\left|\eta_{2}\right|^{2}=\left|\xi_{3}\right|^{2}-\left|\eta_{3}\right|^{2}=\left|\xi_{2}\right|^{2}-\left|\xi_{3}\right|^{2}=0, \\
& M_{5} \subset \mathbb{C}^{8}: \xi_{2} \bar{\xi}_{3}-\bar{\eta}_{2} \eta_{3}=\xi_{3} \bar{\xi}_{4}-\bar{\eta}_{3} \eta_{4}=0 .
\end{aligned}
$$

Note that all these sets contain the $\left(\xi_{1}, \eta_{1}\right)$-subspace. Let $\psi$ be an elliptic holomorphic symplectic transformation of $\mathbb{C}^{2}$ which is not normalizable by any convergent symplectic transformation. For each $M_{j}$, one can find a map $\varphi$ which is the product of $\psi$ with a suitable elliptic $\mathbb{C}$-linear symplectic transformation of $\mathbb{C}^{2 n-2}(n=2,3$, or 4$)$ such that $M_{j}$ is invariant under $\varphi$. However, $\varphi$ is not normalizable by any convergent symplectic transformation. 


\section{Normalization of Levi-flat invariant sets.}

Throughout the paper we denote by $[p]_{k}$ the sum of homogeneous terms of order $k$, whenever $p$ is a multivariable power series.

In Section 2, four special Levi-flat classes of quadrics were introduced: the sets defined by $(2.2),(2.3),(2.8)$ and $(2.12)$, respectively. We now study Levi-flat sets defined by equations whose lowest order terms correspond to these quadratic polynomials, and we try to simplify their equations by changing holomorphic coordinates. For this purpose, we shall combine two singularities (2.2) and (2.3) since such combination results in no extra difficulties. One can also treat two singularities (2.8) and (2.12) simultaneously. Notice that quantities $\mu_{j}$ in Section 2 are symplectic invariants, but are not the holomorphic ones. Since holomorphic coordinates, not the symplectic ones, are used throughout this section, we can restrict ourselves to the case all $\mu_{j}=1$. We shall also use a simpler permutation $\sigma$ to simplify notations.

To combine two types of singularities (2.2) and (2.3), we let $\sigma$ be a permutation of $1, \ldots, m(m \leq n)$ satisfying

$$
\sigma(j)= \begin{cases}j, & \text { if } 1 \leq j \leq K, \\ \sigma^{-1}(j) \neq j, & \text { if } K<j \leq K+2 L=m .\end{cases}
$$

In particular, $\sigma^{2}=$ Id. Put

$$
q_{j}(\xi, \eta)= \begin{cases}\sum_{k=1}^{n} a_{j k} \xi_{k} \eta_{k}, & 1 \leq j \leq K \\ \xi_{j} \eta_{\sigma(j)}, & K<j \leq m\end{cases}
$$

with

$$
\operatorname{det}\left(a_{i j}\right)_{1 \leq i, j \leq K} \neq 0
$$

Define the set

$$
\mathcal{Q} \subset \mathbb{C}^{2 n}: q_{j}(\xi, \eta)+\bar{q}_{\sigma(j)}(\bar{\xi}, \bar{\eta})=0, \quad j=1, \ldots, m .
$$

Note that we allow $K=0$ or $m$, so that sets (2.2) and (2.3) $\left(\right.$ all $\left.\mu_{j}=1\right)$ are indeed special cases of (3.4). In Proposition 3.5 below, we shall prove that (3.4) is generic, irreducible and Levi-flat.

One of main results of this section is the following.

Theorem 3.1. - Let $M \subset \mathbb{C}^{2 n}$ be a real analytic variety defined by

$$
q_{j}(\xi, \eta)+\bar{q}_{\sigma(j)}(\bar{\xi}, \bar{\eta})+R_{j}(\xi, \eta, \bar{\xi}, \bar{\eta})=0, \quad j=1, \ldots, m
$$


where $\sigma, q_{j}$, and $a_{i j}$ are given by (3.1)-(3.3) and $R_{j}$ are real analytic and satisfy the reality condition

$$
R_{\sigma(j)}(\xi, \eta, \bar{\xi}, \bar{\eta})=\bar{R}_{j}(\bar{\xi}, \bar{\eta}, \xi, \eta)
$$

Assume that $M$ is Levi-flat. Then there are $m$ holomorphic functions $H_{j}(\xi, \eta)=O(3)$ such that $M$, as a germ of real analytic set at the origin, is given by

$$
q_{j}(\xi, \eta)+\bar{q}_{\sigma(j)}(\bar{\xi}, \bar{\eta})+H_{j}(\xi, \eta)+\bar{H}_{\sigma(j)}(\bar{\xi}, \bar{\eta})=0, \quad j=1, \ldots, m
$$

To combine two singularities (2.8) and (2.12), let $\sigma$ be still given by (3.1), and put

$$
q_{j}(\xi, \eta, \bar{\xi}, \bar{\eta})= \begin{cases}\left|\xi_{j}\right|^{2}-\left|\eta_{j}\right|^{2}, & 1 \leq j \leq K \\ \xi_{j} \bar{\xi}_{\sigma(j)}-\bar{\eta}_{j} \eta_{\sigma(j)}, & K<j \leq m\end{cases}
$$

We now state the following rigidity result.

Theorem 3.2. - Let $M \subset \mathbb{C}^{2 n}$ be a Levi-flat real analytic variety defined by

$$
q_{j}(\xi, \eta, \bar{\xi}, \bar{\eta})+R_{j}(\xi, \eta, \bar{\xi}, \bar{\eta})=0, \quad 1 \leq j \leq m,
$$

where $\sigma, q_{j}$ are given (3.1) and (3.8), and $R_{j}(\xi, \eta, \bar{\xi}, \bar{\eta})=O(3)$ are real analytic and satisfy the reality condition (3.6). Then near the origin, $M$ is holomorphically equivalent to the set

$$
\mathcal{Q} \subset \mathbb{C}^{2 n}: q_{j}(\xi, \eta, \bar{\xi}, \bar{\eta})=0, \quad j=1, \ldots, m .
$$

Note that (3.10) becomes (2.12) (all $\mu_{j}=1$ and $\sigma=\mathrm{Id}$ ) when $K=0$, or (2.8) (all $\left.\mu_{j}=1\right)$ when $K=m$. As a consequence of Proposition 3.7 we shall see that the set (3.10) is generic, irreducible, and Levi-flat.

The main purpose of this section is to prove Theorem 3.1 and Theorem 3.2. We start with the following.

Lemma 3.3. - Let $V \subset \mathbb{C}^{2 m} \times \mathbb{C}^{n}$ be a complex variety defined by

$$
f_{j}=x_{j}^{2}+y_{j}^{2}+q_{j}(x, y, z)+h_{j}(x, y, z)=0, \quad j=1, \ldots, m,
$$

where $q_{j}$ is a quadratic polynomial in $x_{j+1}, y_{j+1}, \ldots, x_{m}, y_{m}, z$ for $j=$ $1, \ldots, m$, and $h_{j}$ are holomorphic functions of order at least 3 . Let $f$ be a holomorphic function vanishing on $V$. Then, as a germ of holomorphic function at the origin, $f$ admits a decomposition

$$
f=a_{1} f_{1}+a_{2} f_{2}+\cdots+a_{n} f_{n}
$$

TOME 51 (2001), FASCICULE 1 
for some holomorphic functions $a_{j}$ with $\min _{j}\left\{\operatorname{ord} a_{j}\right\}=$ ord $f-2$.

Proof. - Without loss of generality one may assume that $f_{j}$ is a quadratic Weierstrass polynomial in $x_{j}$. Put $f=g_{0}$. Using the Weierstrass division theorem, one gets

$$
g_{j-1}=a_{j} f_{j}+g_{j}, \quad j=1, \ldots, m
$$

with $\min \left\{\operatorname{ord} a_{j}+2\right.$, ord $\left.g_{j}\right\}=\operatorname{ord}\left\{g_{j-1}\right\} \geq \operatorname{ord} f$. Hence

$$
f=a_{1} f_{1}+\cdots+a_{m} f_{m}+g_{m} .
$$

Assuming that $f \not \equiv 0$, we would like to show that ord $g_{m}>$ ord $f$ and $\min \left\{\operatorname{ord} a_{j}\right\}=\operatorname{ord} f-2$.

Consider the dilation $(x, y, z) \rightarrow(\epsilon x, \epsilon y, \epsilon z)$. Let $V^{\epsilon}$ be defined by

$$
x_{j}^{2}+y_{j}^{2}+q_{j}(x, y, z)+h_{j}(x, y, z, \epsilon) \equiv f_{j}(\epsilon x, \epsilon y, \epsilon z) / \epsilon^{2}=0
$$

for $j=1, \ldots, m$. Obviously, $h_{j}=0$ for $\epsilon=0$. Put

$$
\Delta_{r}=\{t \in \mathbb{C}:|t|<r\}
$$

and let $\Delta_{r}^{k}$ be the products of $k$ copies of $\Delta_{r}$. We shall also drop the superscript in $\Delta_{r}^{k}$ whenever the dimension $k$ is clear from context. Let $\pi$ be the projection $(x, y, z) \rightarrow(y, z)$. Choose $\delta, \delta^{\prime}$ so small that the restriction $\pi: V^{\epsilon} \equiv V^{\epsilon} \cap\left(\Delta_{\delta}^{m} \times \Delta_{\delta^{\prime}}^{m+n}\right) \rightarrow \Delta_{\delta^{\prime}}^{m+n}$ is a $2^{m}$-to-1 branched covering, for which the branch locus is denoted by $B^{\epsilon}$. Obviously,

$$
B^{0}=V^{0} \cap\left\{\left(y_{m}^{2}+q_{m}(x, y, z)\right) \cdots\left(y_{1}^{2}+q_{1}(x, y, z)\right)=0\right\}
$$

is a proper subvariety of $V^{0}$; hence, $B^{\epsilon}$ is also a proper subvariety of $V^{\epsilon}$ for small $\epsilon$. Off the branch locus $B^{\epsilon}, V^{\epsilon}$ is locally given by $x=d_{J}(y, z, \epsilon), J \in$ $\mathbb{Z}_{2}^{m}=\{0,1\}^{m}$ with $d_{J}(\cdot, \epsilon) \rightarrow d_{J}(\cdot, 0)$ as $\epsilon \rightarrow 0$. Note that for $\epsilon=0$ the $k$-th coordinate of $d_{J}$ is obtained by choosing a square root of $-y_{k}^{2}-q_{k}(x, y, z)$, starting with $k=m$. This implies that for $J=\left(j_{1}, \ldots, j_{m}\right) \in \mathbb{Z}_{2}^{m}$ and $J_{k}=$ $\left(j_{k}, \ldots, j_{m}\right)$, one has $d_{J}(y, z, 0)=\left(X_{J_{1}}(y, z), X_{J_{2}}(y, z), \ldots, X_{J_{m}}(y, z)\right)$. Moreover,

$$
X_{\left(0, J_{k+1}\right)}(y, z) \neq X_{\left(1, J_{k+1}\right)}(y, z), \quad J_{k+1} \in \mathbb{Z}_{2}^{m-k-1}
$$

for $(y, z) \notin B^{0}$ and $1 \leq k \leq m$.

Assume for the sake of contradiction that ord $g_{m}=$ ord $f \equiv k$. Note that

$$
g_{m}(\epsilon x, \epsilon y, \epsilon z) / \epsilon^{k}=\left[g_{m}\right]_{k}(x, y, z)+\epsilon \tilde{g}_{m}(x, y, z, \epsilon)
$$


vanishes on $V^{\epsilon}$, and that ord $\tilde{g}_{m}>k$. Since $q_{j}$ is independent of $x_{1}, \ldots, x_{j}$, one gets from $\left[g_{j-1}\right]_{k}=\left[a_{j}\right]_{k-2}\left[f_{j}\right]_{2}+\left[g_{j}\right]_{k}$ that

$$
\left[g_{m}\right]_{k}(x, y, z)=\sum_{\alpha \in \mathbb{Z}_{2}^{m}} c_{\alpha}(y, z) x^{\alpha} .
$$

Now (3.12) yields

$$
\sum_{\alpha \in \mathbb{Z}_{2}^{m}} c_{\alpha}(y, z) d_{J}^{\alpha}(y, z, \epsilon)+\epsilon \tilde{g}_{m}\left(d_{J}(y, z, \epsilon), y, z, \epsilon\right)=0, \quad J \in \mathbb{Z}_{2}^{m}
$$

for $(y, z) \notin \pi\left(B^{\epsilon}\right)$. Letting $\epsilon \rightarrow 0$, one gets

$$
\sum_{\alpha \in \mathbb{Z}_{2}^{m}} c_{\alpha}(y, z) d_{J}^{\alpha}(y, z, 0)=0, \quad J \in \mathbb{Z}_{2}^{m}
$$

for $(y, z) \notin \pi\left(B^{0}\right)$. The above are $2^{m}$ linear equations in $2^{m}$ unknowns $c_{\alpha}(y, z)$, for which the coefficients $d_{J}^{\alpha}(y, z), \alpha, J \in \mathbb{Z}_{2}^{m}$ form a block matrix

$$
D=\left(\begin{array}{cc}
D^{\prime} & D^{\prime} \\
X_{0} D^{\prime} & X_{1} D^{\prime}
\end{array}\right)
$$

where $D^{\prime}$ is the matrix formed by $d_{\left(0, J^{\prime}\right)}^{\left(0, \alpha^{\prime}\right)}$ with $\alpha^{\prime}, J^{\prime} \in \mathbb{Z}_{2}^{m-1}$, and $X_{0}, X_{1}$ are diagonal $2^{m-1} \times 2^{m-1}$ matrices of which the $J^{\prime}$-th diagonal entry is $X_{\left(0, J^{\prime}\right)}(y, z)$ and $X_{\left(1, J^{\prime}\right)}(y, z)$, respectively. From (3.11) it follows that $\operatorname{det}\left(X_{0}-X_{1}\right) \neq 0$. Applying induction on $m$, one gets $\operatorname{det} D=\operatorname{det}\left(X_{0}-X_{1}\right)$ $\operatorname{det} D^{\prime} \neq 0$. Therefore, $c_{\alpha}=0$. This shows that ord $g_{m}>\operatorname{ord} f$ and ord $f=\min \left\{\operatorname{ord} a_{j}\right\}+2$.

However, $g_{m}$ still vanishes on $V$, so one can find another decomposition for $g_{m}$. Inductively, one can at least achieve

$$
f=a_{1} f_{1}+\cdots+a_{m} f_{m}, \quad \min \left\{\operatorname{ord} a_{m}\right\}=\operatorname{ord} f-2
$$

for some formal power series $a_{j}$. By a theorem of M. Artin [1], there are convergent power series $\tilde{a}_{j}(x, y, z)=a_{j}(x, y, z)+O(k)$ satisfying (3.13). This completes the proof of the lemma.

Lemma 3.4. - Let $V, q_{1}, \ldots, q_{m}$ be as in Lemma 3.3, and let $q_{j}(0,0, z)=\sum_{k=1}^{n} a_{j k} z_{k}^{2}$. Assume that the rank of $\left(a_{j k}\right)$ is $m$. Then $V$ is irreducible.

Proof. - As in the proof of Lemma 3.3, let $\pi$ be the projection $(x, y, z) \rightarrow(y, z)$. Choose $\delta, \delta^{\prime}>0$ such that the restriction $\pi: V \equiv$ $V \cap\left(\Delta_{\delta}^{m} \times \Delta_{\delta^{\prime}}^{m+n}\right) \rightarrow \Delta_{\delta^{\prime}}^{m+n}$ is a $2^{m}$-to-1 branched covering, for which the 
set of points of branch order $2^{m}$, i.e., the points $p \in V$ with $\pi^{-1} \pi(p)=\{p\}$, contains a subset $B$ satisfying additional equations

$$
2 x_{j}+\partial_{x_{j}} h_{j}=\partial_{x_{j}} f_{j}=0, \quad j=1, \ldots, m .
$$

For a possible smaller $\delta^{\prime}$, the above equations define a one-to-one covering over $\Delta_{\delta^{\prime}}^{m+n}$. Solving for $x$ from $(3.14)$ and inserting $x$ into $f_{j}(x, y, z)$ yields new functions

$$
\tilde{f}_{j}(y, z)=y_{j}^{2}+q_{j}(0, y, z)+\tilde{h}_{j}(y, z)=0, \quad j=1, \ldots, m
$$

on $B$. Since $\left(a_{j k}\right)$ is of rank $m$, one may assume that $\left(a_{j k}\right)_{1 \leq j, k \leq m}$ is nonsingular. Fix $\left(y^{0}, z^{0}\right)$ on $y_{j}^{2}+q_{j}(0, y, z)=0, j=1, \ldots, m$, so that $z_{m+1}^{0}=\cdots=z_{n}^{0}=0$, while none of $y_{1}^{0}, z_{1}^{0} \ldots, y_{m}^{0}, z_{m}^{0}$ is zero. Consider the dilation $g_{j}(y, z, \epsilon) \equiv \tilde{f}_{j}(\epsilon y, \epsilon z) / \epsilon^{2}$. Then $\partial g / \partial\left(z_{1}, \ldots, z_{m}\right)$ is nonsingular for $(y, z)=\left(y^{0}, z^{0}\right)$ and $\epsilon=0$. By the implicit function theorem, one can verify that for small $\epsilon$, there exist $x(\epsilon), z(\epsilon)$ such that $x(\epsilon), \epsilon y^{0}, z(\epsilon)$ satisfy (3.14)(3.15) and $|x(\epsilon)|,|z(\epsilon)| \leq c \epsilon$ for some constant $c\left(z_{j}(\epsilon)=0\right.$ for $\left.j>m\right)$.

On the other hand, the singular locus $V_{s}$ of $V$ is contained in

$$
\operatorname{det} \partial f / \partial y=2^{m} y_{1} y_{2} \cdots y_{m}+O(m+1)=0,
$$

which obviously does not contain $\left(x(\epsilon), \epsilon y^{0}, z(\epsilon)\right) \in B$ for small $\epsilon \neq 0$. This shows that $V$ contains a smooth point of branch order $2^{m}$. Hence, $V^{*}$ is connected, i.e., $V$ is irreducible. This completes the proof of the lemma.

Note that the above proof is based on branched coverings and the existence of smooth branch points of maximal branch order. Applying the same argument to the family of complex varieties

$$
V^{\epsilon}: f_{j}^{\epsilon}(x, y, z) \equiv f_{j}(\epsilon x, \epsilon y, \epsilon z) / \epsilon^{2}, \quad j=1, \ldots, m
$$

yields that any neighborhood of the origin contains another neighborhood $D$, independent of $\epsilon$, such that each $V^{\epsilon} \cap D$ is connected for small $\epsilon$.

We now apply the above lemmas to the real analytic variety given by (3.5) and (3.6).

Proposition 3.5. - Let $M \subset \mathbb{C}^{2 n}$ be a real analytic set given by (3.5)-(3.6). Then $M$ is a generic and irreducible real analytic variety of codimension $m$. Also, a germ of any real analytic function $f$ vanishing on an open subset of $M^{*}$, of which the closure contains the origin, can be decomposed into $a_{1} r_{1}+\cdots+a_{m} r_{m}$ for germs of some complex-valued real analytic functions $a_{j}$ with $\min _{j}\left\{\right.$ ord $\left.a_{j}\right\}=$ ord $f-2$. Moreover, the set $\mathcal{Q}$, defined by (3.4), is Levi-flat. 
Proof. - Put $r_{j}^{\epsilon}(\xi, \eta, \bar{\xi}, \bar{\eta})=r_{j}(\epsilon \xi, \eta, \epsilon \bar{\xi}, \epsilon \bar{\eta}) / \epsilon^{2}$ for $j=1, \ldots, m$. Note that $\mathcal{Q}$ is defined by $r_{1}^{0}=\cdots=r_{m}^{0}=0$. At $\xi=(0, \ldots, 0), \eta=$ $(1, \ldots, 1)$, one has

$$
\operatorname{det} \partial r^{0} / \partial\left(\xi_{1}, \ldots, \xi_{m}\right)=\operatorname{det} A \neq 0, \quad A=\left(a_{i j}\right)_{1 \leq i, j \leq K} .
$$

This shows that $\operatorname{dim} \mathcal{Q} \geq 4 n-m$, and that $\mathcal{Q}$ is generic if $\operatorname{dim} \mathcal{Q}=$ $4 n-m$. Now the implicit function theorem implies that $M$ enjoys the same property.

To show that $\operatorname{dim} M=4 n-m$, we consider the complex variety

$$
\mathcal{M} \subset \mathbb{C}^{2 n} \times \mathbb{C}^{2 n}: r_{j}(\xi, \eta, \bar{\xi}, \bar{\eta})=\bar{r}_{j}(\bar{\xi}, \bar{\eta}, \xi, \eta)=0, \quad j=1, \ldots, m,
$$

where $\xi, \eta, \bar{\xi}, \bar{\eta}$ are now independent complex variables. By the reality conditions $(3.6), \mathcal{M}$ is actually defined by $r_{j}(\xi, \eta, \bar{\xi}, \bar{\eta})=0$ for $j=1, \ldots, m$; more explicitly, $\mathcal{M}$ is given by

$$
\begin{aligned}
\xi_{j} \eta_{j}+\sum_{k=K+1}^{n} b_{j k} \xi_{k} \eta_{k}+\sum_{k=1}^{n} c_{j k} \bar{\xi}_{k} \bar{\eta}_{k}+\tilde{R}_{j}(\xi, \eta, \bar{\xi}, \bar{\eta})=0, \quad 1 \leq j \leq K, \\
\xi_{j} \eta_{\sigma(j)}+\bar{\xi}_{\sigma(j)} \bar{\eta}_{j}+R_{j}(\xi, \eta, \bar{\xi}, \bar{\eta})=0, \quad K<j \leq m,
\end{aligned}
$$

where $\left(c_{j k}\right)_{1 \leq j, k \leq K}$ is nonsingular. Choose complex linear coordinates $x, y, z$ so that

$$
\begin{array}{lll}
\xi_{j} \eta_{j}=x_{j}^{2}+y_{j}^{2}, & \bar{\xi}_{j} \bar{\eta}_{j}=z_{j}^{2}+z_{m+j}^{2}, & 1 \leq j \leq K, \\
\xi_{j} \eta_{\sigma(j)}=x_{j}^{2}+y_{j}^{2}, & \bar{\xi}_{\sigma(j)} \bar{\eta}_{j}=z_{j}^{2}+z_{m+K+j}^{2}, & K<j \leq m .
\end{array}
$$

Substitute the rest of $z$-variables for $\xi, \eta, \bar{\xi}, \bar{\eta}$ that do not appear in (3.16). Obviously, in the new coordinates, $\mathcal{M}$ has defining functions satisfying all the assumptions in Lemma 3.4; hence, $\mathcal{M}$ is irreducible and of codimension $m$. Since $M \equiv\{(\xi, \eta, \bar{\xi}, \bar{\eta}):(\xi, \eta) \in M\} \subset \mathcal{M}$ is of real dimension at least $4 n-m$, then $\mathcal{M}$ is the Bruhat-Whitney-Cartan complexification of $M$. A result of H. Cartan [7] implies that the complexification of $f$ vanishes on $\mathcal{M}$. In view of Lemma 3.3, the complexification of $f$, and hence $f$, admits a decomposition $a_{1} r_{1}+\cdots+a_{m} r_{m}$.

The above argument shows especially that $\mathcal{Q}$ is irreducible, generic, and of codimension $m$. Finally, we want to show that $\mathcal{Q}$ is Levi-flat. Note that $\mathcal{Q} \backslash \cup_{j=1}^{m}\left\{\eta_{j}=0\right\}$ is the disjoint union of graphs over the $\left(\xi_{m+1}, \ldots, \xi_{n}, \eta\right)$-space, which are given implicitly by

$$
\begin{gathered}
\sum_{k=1}^{n} a_{j k} \xi_{k} \eta_{k}=i t_{j}, \quad \sigma(j)=j, \\
\xi_{j} \eta_{\sigma(j)}=t_{j}+i t_{\sigma(j)}, \quad \xi_{\sigma(j)} \eta_{j}=-\left(t_{j}-i t_{\sigma(j)}\right), \quad \sigma(j)>j
\end{gathered}
$$

TOME 51 (2001), FASCICULE 1 
with $t_{j} \in \mathbb{R}$. Hence, $\mathcal{Q} \backslash \cup_{j=1}^{m}\left\{\eta_{j}=0\right\}$ is smooth and Levi-flat. Since $Q_{c}$ is generic, it is contained in the closure of $\mathcal{Q} \backslash \cup_{j=1}^{m}\left\{\xi_{j}=0\right\}$. Therefore, $\mathcal{Q}_{c}$ is Levi-flat. This completes the proof of the proposition.

Before we prove the first main result of this section, we should recall the Segre varieties associated to a real variety [15]. Let $M$ be a germ of real analytic variety at $0 \in \mathbb{C}^{n}$ defined by $r_{1}(z, \bar{z})=\cdots=r_{m}(z, \bar{z})=0$, where $r_{j}$ are real power series convergent on a polydisc $\Delta_{\epsilon}^{n} \times \Delta_{\epsilon}^{n} \subset \mathbb{C}^{n} \times \mathbb{C}^{n}$. Then the Segre varieties are the family of complex varieties

$$
Q_{w}=\left\{z \in \Delta_{\epsilon}^{n}: r_{1}(z, \bar{w})=\cdots=r_{m}(z, \bar{w})=0\right\}, \quad w \in \Delta_{\epsilon}^{n} .
$$

For a fixed $w \in \Delta_{\epsilon}^{n}$, the Segre variety $Q_{w}$ could be an empty set, or the whole polydisc $\Delta_{\epsilon}^{n}$, or a proper subvariety of dimension between 0 and $n-1$. For our applications of Segre varieties, we shall only deal with real analytic sets $M$ that admit defining functions $r_{1}, \ldots, r_{m}$ such that germs of real analytic function vanishing on $M$ are always generated by $r_{1}, \ldots, r_{m}$. This implies that the Segre varieties $Q_{w}$ are independent of the choice of such defining functions $r_{j}$, except that a possible smaller polydisc $\Delta_{\epsilon}^{n} \times \Delta_{\epsilon}^{n}$ has to be chosen for a given set of such defining functions. Also, note that, given two real analytic varieties $M, M^{\prime}$ admitting such defining functions and a biholomorphic mapping $\phi$ sending $M$ onto $M^{\prime}, \phi$ must send Segre varieties of $M$ into Segre varieties of $M^{\prime}$ (by shrinking the polydiscs on which $M, M^{\prime}$ are defined if necessary). Finally, we should mention that if a germ of complex variety $V$ at $w$ is contained in $M$, then $V$ is also contained in the Segre variety $Q_{w}$ of $M$.

Proof of Theorem 3.1. - Following [6], we start with the dilation $M^{\epsilon}$ defined by

$$
r_{j}^{\epsilon}(\xi, \eta, \bar{\xi}, \bar{\eta}) \equiv r_{j}(\epsilon \xi, \epsilon \eta, \epsilon \bar{\xi}, \epsilon \bar{\eta}) / \epsilon^{2}=0, \quad j=1, \ldots, m
$$

Write

$$
r_{j}^{\epsilon}(\xi, \eta, \bar{\xi}, \bar{\eta})=q_{j}(\xi, \eta, \bar{\xi}, \bar{\eta})+R_{j}(\xi, \eta, \bar{\xi}, \bar{\eta}, \epsilon) .
$$

Parameterize

$$
\gamma^{\epsilon}=M^{\epsilon} \cap\left\{\eta_{j}=1, \quad j \leq m ; \quad \xi_{j}=\eta_{j}=0, \quad j>m\right\}
$$

by $z=\gamma^{\epsilon}(t)=(\xi(t), \eta(t))\left(t \in \mathbb{R}^{m}\right)$ with

$$
\begin{aligned}
& \sum_{k=1}^{m} a_{j k} \xi_{k}(t)=i t_{j}+\alpha_{j}(t, \epsilon), \quad \alpha_{j}=\bar{\alpha}_{j} \quad \text { if } \sigma(j)=j, \\
& \left.\begin{array}{r}
\xi_{j}(t)=t_{j}+i t_{\sigma(j)}+\alpha_{j}(t, \epsilon) \\
\xi_{\sigma(j)}(t)=-\left(t_{j}-i t_{\sigma(j)}-\bar{\alpha}_{j}(t, \epsilon)\right)
\end{array}\right\} \text { if } \sigma(j)>j .
\end{aligned}
$$

ANNALES DE L'INSTITUT FOURIER 
Then $\alpha_{j}$ should satisfy

$$
2 \alpha_{j}+R_{j}(\xi(t), \eta(t), \bar{\xi}(t), \bar{\eta}(t), \epsilon)=0, \quad \sigma(j) \geq j
$$

The implicit function theorem implies that the above equations have a unique solution

$$
\alpha_{j}=\alpha_{j}(t, \epsilon), \quad \sigma(j) \geq j
$$

with $\left.\alpha_{j}\right|_{\epsilon=0} \equiv 0$.

The Segre variety $Q_{\gamma^{\epsilon}(t)}$ of $M^{\epsilon}$ is defined by

$$
\begin{aligned}
& -i t_{j}+\alpha_{j}(t, \epsilon)+\sum_{k=1}^{n} a_{j k} \xi_{k} \eta_{k}+R_{j}=0 \quad \text { if } \sigma(j)=j, \\
& \left.\begin{array}{rl}
-t_{j}-i t_{\sigma(j)}+\alpha_{j}(t, \epsilon)+\xi_{j} \eta_{\sigma(j)}+R_{j} & =0 \\
t_{j}-i t_{\sigma(j)}+\bar{\alpha}_{j}(t, \epsilon)+\xi_{\sigma(j)} \eta_{j}+R_{\sigma(j)} & =0
\end{array}\right\} \quad \text { if } \sigma(j)>j
\end{aligned}
$$

for $R_{j}=R_{j}\left(\xi, \eta, \bar{\gamma}^{\epsilon}(t), \epsilon\right)$. By applying the fixed-point theorem, one can solve (3.17) to get

$$
t_{j}=i h_{j}(\xi, \eta, \epsilon), \quad j=1, \ldots, m,
$$

where $h_{j}$ are holomorphic functions of $\xi, \eta, \epsilon$. It is easy to see that

$$
h_{j}(\xi, \eta, 0)=\sum_{k=1}^{m} c_{j k} q_{k}(\xi, \eta), \quad \operatorname{det}\left(c_{j k}\right) \neq 0 .
$$

This implies that for small $\epsilon$, all $h_{j}(\xi, \eta, \epsilon)$ are well-defined on the domain

$$
D_{s}=\Delta_{2}^{2 n} \cap\left\{\left|q_{j}(\xi, \eta)\right|<s, j=1, \ldots, m\right\},
$$

where $s>0$ is small and independent of $\epsilon$. Clearly, $\gamma^{\epsilon}(t)$ is contained in $D_{s}$ when $\epsilon, t$ are small. As mentioned after the proof of Lemma 3.4, $D_{s}$ contains a neighborhood $D^{\prime}$ of the origin such that for each small $\epsilon$ the smooth locus of $\mathcal{M}^{\epsilon} \cap D^{\prime}$ is connected.

Note that $\partial\left(r_{1}^{\epsilon}, \ldots, r_{m}^{\epsilon}\right) / \partial\left(\xi_{1}, \ldots, \xi_{m}\right)$ is nonsingular near $\gamma^{\epsilon}$. Hence, $M^{\epsilon}$ is smooth and of $C R$-dimension $2 n-m$ near $\gamma^{\epsilon}$. This implies that $M^{\epsilon}$ is Levi-flat near $\gamma^{\epsilon}$; in particular, the branch $Q_{\gamma^{\epsilon}(t)}^{\prime}$ of $Q_{\gamma^{\epsilon}(t)}$, which passes through $\gamma^{\epsilon}(t)$, is contained in $M^{\epsilon}$.

Next, we would like to show that $Q_{\gamma^{\epsilon}(t)}^{\prime} \subset M^{\epsilon}$ sweep out an open subset of $\left(M^{\epsilon}\right)^{*} \cap D^{\prime}$. To this end we take a polydisc $\Delta_{s^{\prime}}^{2 n} \subset D^{\prime}$. Rewrite $(3.17)$ as

$$
\xi_{j}=\eta_{j}^{-1}\left(\sum_{k=1}^{K} b_{j k}\left(i t_{k}-\alpha_{k}-R_{k}\right)+\sum_{k>K} b_{j k} \xi_{k} \eta_{k}\right), \quad \sigma(j)=j,
$$




$$
\left.\begin{array}{r}
\xi_{j}=\eta_{\sigma(j)}^{-1}\left(t_{j}+i t_{\sigma(j)}-\alpha_{j}-R_{j}\right) \\
\xi_{\sigma(j)}=\eta_{j}^{-1}\left(\left(i t_{\sigma(j)}-t_{j}-\bar{\alpha}_{j}\right)-R_{\sigma(j)}\right)
\end{array}\right\} \quad \sigma(j)>j .
$$

Applying the fixed-point theorem to the above equations, one can get a unique solution $\xi_{j}=p_{j}^{\epsilon}\left(\eta, \xi^{\prime \prime}, t\right)$ defined on $E^{m} \times \Delta_{s^{\prime \prime}}^{2 n-2 m}$ for $|t|<s^{\prime \prime}$, where $s^{\prime \prime}<s^{\prime}$ is a positive constant depending only on $s^{\prime}$ and

$$
E=\left\{w \in \mathbb{C}: s^{\prime} / 2<|w|<3 / 2,|\arg w|<\pi / 2\right\} .
$$

This shows that when $\epsilon, t$ are sufficiently small, the branch $Q_{\gamma^{\epsilon}(t)}^{\prime}$ contains the graph

$$
G_{t}^{\epsilon} \subset \mathbb{C}^{2 n}: \xi_{j}=p_{j}^{\epsilon}\left(\xi^{\prime \prime}, \eta, t\right), \quad\left(\eta, \xi^{\prime \prime}\right) \in E^{m} \times \Delta_{s^{\prime \prime}}^{2 n-2 m} .
$$

Obviously, $G_{t}^{\epsilon} \cap \Delta_{s^{\prime}}^{2 n}$ is nonempty if $\epsilon, t$ are small, and $\left(\partial p^{\epsilon} / \partial t\right)\left(\eta, \xi^{\prime \prime}, t\right)$ is nonsingular on $E^{m} \times \Delta_{s^{\prime \prime}}^{2 n-2 m+1}$ for $\epsilon=0$. This shows that $G^{\epsilon} \equiv \cup G_{t}^{\epsilon} \subset M^{\epsilon}$ is an immersed real manifold of dimension $2 n-m$. Thus, $G^{\epsilon} \cap\left(M^{\epsilon}\right)^{*} \cap \Delta_{s^{\prime}}^{2 n}$ is nonempty. On the other hand, $G_{t}^{\epsilon}$ is contained in the Segre variety $Q_{\gamma^{\epsilon}(t)}^{\prime}$ on which the holomorphic functions $h_{j}(\xi, \eta, \epsilon)$ are pure imaginary. Therefore, all $\Re\left\{h_{j}(\xi, \eta, \epsilon)\right\}$ vanish on a nonempty open subset of $\left(M^{\epsilon}\right)^{*} \cap \Delta_{s^{\prime}}^{2 n}$. The latter is, however, a totally real subspace of $\left(\mathcal{M}^{\epsilon}\right)^{*} \cap D$ of maximal dimension. Therefore, the complexification of each $\Re\left\{h_{j}(\xi, \eta, \epsilon)\right\}$ vanishes on $\mathcal{M}^{\epsilon} \cap D$. In return, $\Re\left\{h_{j}(\xi, \eta, \epsilon)\right\}$ vanishes on $M^{\epsilon}$.

We now fix a small $\epsilon$. In view of Proposition 3.5, we have

$$
\Re\left\{h_{j}(\xi, \eta, \epsilon)\right\}=\sum d_{j k}(\xi, \eta, \bar{\xi}, \bar{\eta}) r_{k}^{\epsilon}(\xi, \eta, \bar{\xi}, \bar{\eta}), \quad j=1, \ldots, m .
$$

In particular, $h_{j}(\xi, \eta, \epsilon)-h_{j}(0,0, \epsilon)=O\left(|(\xi, \eta)|^{2}\right)$. ¿From (3.18), we know that $\left(d_{j k}\right)(0)$ is invertible. Let $\left(d_{j k}^{\prime}\right)$ be the inverse of the constant matrix $\left(d_{j k}\right)(0)$. Put

$$
h_{j}^{*}(\xi, \eta)=\sum_{k=1}^{m} d_{j k}^{\prime} h_{k}(\xi, \eta, \epsilon) .
$$

Then $q_{j}$ is the quadratic form of $h_{j}^{*}$. Notice that the right-hand side of (3.19) is real; in particular, its quadratic form is real also. Hence, $d_{j k}(0)=\bar{d}_{j \sigma(k)}(0)$. From $\sigma^{2}=\mathrm{Id}$, it follows that $d_{j k}^{\prime}=\bar{d}_{\sigma(j) k}^{\prime}$. On $M^{\epsilon}$, we have $\bar{h}_{j}(\bar{\xi}, \bar{\eta}, \epsilon)=-h_{j}(\xi, \eta, \epsilon)$. Now a straightforward computation shows that $h_{j}^{*}(\xi, \eta, \epsilon)=-\bar{h}_{\sigma(j)}^{*}(\xi, \eta, \epsilon)$ on $M^{\epsilon}$. This shows that $M^{\epsilon}$, and hence $M$, is of the form (3.7). The proof of the theorem is complete.

We now turn to the proof of Theorem 3.2. The rest of the section is to show that the Levi-flat analytic set defined by (3.9) is holomorphically equivalent to the set (3.10). 
We shall use general holomorphic coordinates for the rest of section. For convenience, we set

$$
z=(x, y), \quad x^{\prime}=\left(x_{1}, \ldots, x_{m}\right), \quad x^{\prime \prime}=\left(x_{m+1}, \ldots, x_{n}\right), \text { etc },
$$

where $(x, y)=\left(x_{1}, \ldots, x_{n}, y_{1}, \ldots, y_{n}\right)$ are coordinates of $\mathbb{C}^{2 n}$. In particular, the last $m-K$ equations in (3.9) are equivalent to the last $m-K$ ones of

$$
r_{j}=x_{j} \bar{y}_{\sigma(j)}+\bar{x}_{\sigma(j)} y_{j}+R_{j}(z, \bar{z})=0, \quad j=1, \ldots, m .
$$

The first $K$ equations in (3.9) are transformed into the first $K$ ones of (3.20) by the transformation

$$
\xi_{j}=(1+i) x_{j}+y_{j}, \quad \eta_{j}=(1+i) x_{j}+i y_{j}, \quad j=1, \ldots, K .
$$

Thus, $M$ is given by (3.20) with $\sigma$ of the form (3.1), and the reality condition (3.6) now reads

$$
R_{\sigma(j)}(z, \bar{z})=\bar{R}_{j}(\bar{z}, z) .
$$

Thus, Theorem 3.2 is reduced to the following.

Theorem 3.6. - Let $M \subset \mathbb{C}^{2 n}$ be a Levi-flat analytic set given by (3.20)-(3.21). Then $M$ is holomorphically equivalent to the set

$$
\mathcal{Q} \subset \mathbb{C}^{2 n}: x_{j} \bar{y}_{\sigma(j)}+\bar{x}_{\sigma(j)} y_{j}=0, \quad j=1,2, \ldots, m .
$$

We need the following.

Proposition 3.7. - Let $M \subset \mathbb{C}^{2 n}$ be a real analytic set defined by (3.20)-(3.21) with $\sigma$ of the form (3.1). Then $M$ is generic, irreducible and of codimension $m$. Also, a germ of any real analytic function $f$ vanishing on a topological component of $M$, of which the closure contains the origin, admits a decomposition $a_{1} r_{1}+\cdots+a_{m} r_{m}$ for some complex-valued real analytic functions $a_{j}$ with $\min \left\{\operatorname{ord} a_{j}\right\}=\operatorname{ord} f-2$. Moreover, the set $\mathcal{Q}$, defined by (3.22), is Levi-flat.

Proof. - The proof is almost the same as that of Proposition 3.5. Take a point $z^{0}=\left(x^{0}, y^{0}\right)$ on $\mathcal{Q}$ such that $x_{j}^{0} \neq 0 \neq y_{j}^{0}$ for $j=1, \ldots, m$. Then $\partial\left(q_{1}, \ldots, q_{m}\right) / \partial\left(x_{1}, \ldots, x_{m}\right) \neq 0$ at $z^{0}$. This shows that $\mathcal{Q}$ has codimension at most $m$, and that $\mathcal{Q}$ is generic if $\operatorname{codim} \mathcal{Q}=m$. By the implicit function theorem, one then knows that $M$ has codimension at most $m$, and it is generic if the codimension is $m$. Using Lemma 3.4, one shows that the complexification of $M$ is the irreducible complex variety of codimension $m$ given by $r_{j}(z, \bar{z})=0, j=1, \ldots, m$. Since $M^{*}$ is a totally 
real subspace in $(\mathcal{M})^{*}$ of maximal dimension, the complexification of $f$ vanishes on $\mathcal{M}$. Lemma 3.3 says that the complexification, and hence $f$, can be decomposed into $a_{1} r_{1}+\cdots+a_{m} r_{m}$ for some real analytic functions $a_{j}$.

Finally, $\mathcal{Q} \backslash \cup_{j=1}^{m}\left\{x_{j}=0\right\}$ is the disjoint union of complex manifolds

$$
y_{j}=i t_{j} x_{j}, \quad 1 \leq j \leq m
$$

with parameters $t_{j} \in \mathbb{C}$ satisfying $t_{\sigma(j)}=\bar{t}_{j}$. Therefore, $\mathcal{Q}$ is Levi-flat. The proof of Proposition 3.7 is complete.

Lemma 3.8. - Let $\sigma$ be a permutation of $1, \ldots, m$, and let $l_{1}, \ldots, l_{m}$ be $\mathbb{C}$-linear functions on $\mathbb{C}^{2 n}$ satisfying

$$
x_{j} \overline{l_{\sigma(j)}(x, y)}+\bar{x}_{\sigma(j)} l_{j}(x, y)=0 .
$$

Then $l_{j}(x, y)=c_{j} x_{j}$ and

$$
c_{\sigma(j)}+\bar{c}_{j}=0 .
$$

If $c, d \in \mathbb{C}^{m}$ satisfy (3.24) with $c-d \in\left(\mathbb{C}^{*}\right)^{m}$, equations

$$
x_{j}^{*}=y_{j}-c_{j} x_{j}, \quad y_{j}^{*}=\left(y_{j}-d_{j} x_{j}\right) /\left(c_{j}-d_{j}\right), \quad 1 \leq j \leq m
$$

define a nonsingular linear transformation with

$$
x_{j}^{*} \bar{y}_{\sigma(j)}^{*}+\bar{x}_{\sigma(j)}^{*} y_{j}^{*}=x_{j} \bar{y}_{\sigma(j)}+\bar{x}_{\sigma(j)} y_{j} .
$$

Proof. - By (3.23) it is clear that $l(x, y)=\left(c_{1} x_{1}, \ldots, c_{m} x_{m}\right)$ with $c_{j}$ satisfying (3.24). Now, $c-d \in\left(\mathbb{C}^{*}\right)^{m}$ implies that the transformation (3.25) is nonsingular. In view of (3.24), the computation for (3.26) is straightforward. The details are left to the reader.

Proposition 3.9. - Let $M \subset \mathbb{C}^{2 n}$ be a Levi-flat real analytic set defined by (3.20)-(3.21). Then in suitable holomorphic coordinates, $M$ is given by

$$
x_{j} \bar{y}_{\sigma(j)}+\bar{x}_{\sigma(j)} y_{j}+R_{j}(z, \bar{z})=0, \quad j=1, \ldots, m
$$

with

$$
\begin{aligned}
R_{j}(z, \bar{z})= & \sum_{k, l=1}^{m} x_{k} \bar{y}_{l} a_{j k l}(z, \bar{z})+\sum_{k, l=1}^{m} \bar{x}_{k} y_{l} \bar{a}_{\sigma(j) k l}(\bar{z}, z) \\
& +\sum_{k, l=1}^{m} x_{k} y_{l} b_{j k l}\left(z, \bar{z}^{\prime \prime}\right)+\sum_{k, l=1}^{m} \bar{x}_{k} \bar{y}_{l} \bar{b}_{\sigma(j) k l}\left(\bar{z}, z^{\prime \prime}\right),
\end{aligned}
$$




$$
\begin{gathered}
b_{j k l}\left(z, 0^{\prime \prime}\right) \equiv b_{\sigma(j) k l}\left(\bar{z}, 0^{\prime \prime}\right) \equiv 0 \\
a_{j k \sigma(j)}(z, 0) \equiv a_{j j l}(0, \bar{z}) \equiv 0 .
\end{gathered}
$$

Proof. - Put $r_{j}^{\epsilon}(z, \bar{z})=r_{j}(\epsilon z, \epsilon \bar{z}) / \epsilon^{2}$ for $\epsilon>0$. Let $M^{\epsilon}$ be the dilation of $M$ defined by $r_{1}^{\epsilon}=\cdots=r_{m}^{\epsilon}=0$. Set

$$
E^{\epsilon}: \partial r_{1}^{\epsilon} \wedge \cdots \wedge \partial r_{m}^{\epsilon} \neq 0
$$

Obviously, $M^{0} \cap E^{0}$ contains an $m$-dimensional totally real submanifold parameterized by

$$
\begin{gathered}
x^{\prime}=(1, \ldots, 1), \quad x^{\prime \prime}=y^{\prime \prime}=(0, \ldots, 0), \\
y_{j}=y_{j}^{0}(t)= \begin{cases}i t_{j}, & \sigma(j)=j, \\
t_{j}+i t_{\sigma(j)} \equiv-\bar{y}_{\sigma(j)}, & \sigma(j)>j\end{cases}
\end{gathered}
$$

for $t_{j} \in \mathbb{R}$. By the implicit function theorem, $M^{\epsilon} \cap E^{\epsilon}$ also contains an $m$-dimensional totally real submanifold $\gamma^{\epsilon}(t)$ parameterized by (3.31) and

$$
y_{j}=y_{j}^{\epsilon}(t)=y^{0}(t)+\alpha_{j}(t, \epsilon), \quad-1<t_{j}<1, \quad j=1, \ldots, m,
$$

where $\alpha_{j}(t)$ are real analytic functions in $t$ and $\epsilon$, satisfying $\alpha_{\sigma(j)}(t, \epsilon)=$ $\bar{\alpha}_{j}(t, \epsilon)$. The Segre variety $Q_{\gamma^{\epsilon}(t)}$ is defined by

$$
y_{j}+x_{j} \bar{y}_{\sigma(j)}(t)+R_{j}\left(z, \gamma^{\epsilon}(t), \epsilon\right)=0, \quad j=1, \ldots, m .
$$

Note that $R_{j} \equiv 0$ when $\epsilon=0$. By the fixed-point theorem, $Q_{\gamma^{\epsilon}(t)} \equiv$ $Q_{\gamma^{\epsilon}(t)} \cap\left\{\left|x_{j}\right|<2,\left|y_{j}\right|<1 / 2\right\}$ is a graph of the form

$$
y_{j}=h_{j}^{\epsilon}\left(x, y^{\prime \prime}, t\right), \quad\left|x_{j}\right|<2, \quad j=1, \ldots, m
$$

for small $\epsilon$ and $t$. In particular, $Q_{\gamma^{\epsilon}(t)}$ is contained in $M^{\epsilon}$.

We would like to show that $0 \in Q_{\gamma^{\epsilon}(t)}$. From (3.32), it follows that for small $\epsilon$ and generic $t, Q_{\gamma^{\epsilon}(t)}$ intersects a fixed $Q_{\gamma^{\epsilon}\left(t_{0}\right)}$ transversely along $S=Q_{\gamma^{\epsilon}(t)} \cap Q_{\gamma^{\epsilon}\left(t_{0}\right)}$. Fix a generic $t$. $S$ contains points which can be arbitrarily close to the origin as $\epsilon$ tends to 0 , and near the origin $S$ is a closed complex submanifold of dimension $2 n-2 m$. To show that $0 \in Q_{\gamma^{\epsilon}(t)}$ it suffices to verify that $S$ contains the origin. Since $Q_{\gamma^{\epsilon}(t)}$ and $Q_{\gamma^{\epsilon}\left(t_{0}\right)}$ are complex submanifolds of codimension $m \leq n$, the span of their tangent spaces at each point in $S$ is the whole space $\mathbb{C}^{2 n}$. Therefore, the differentials $d r_{j}$ vanish on $S$. On the other hand, we have

$$
\partial_{x_{j}} r_{j}=\bar{y}_{\sigma(j)}+O\left(|z|^{2}\right), \quad \partial_{y_{j}} r_{j}=\bar{x}_{\sigma(j)}+O\left(|z|^{2}\right), \quad j=1, \ldots, m .
$$

Near the origin the above equations define a (connected) smooth real submanifold $S^{\prime}$ of dimension $2 n-2 m$. Obviously, $S^{\prime}$ contains the origin. 
Since $S\left(\subset S^{\prime}\right)$ and $S^{\prime}$ have the same real dimension and both are closed sets, they must coincide near the origin. This shows that $S$, and hence $Q_{\gamma^{\epsilon}(t)}$, contains 0. Notice that the union of $Q_{\gamma^{\epsilon}(t)}$ contains an open subset $U$ of $\left(M^{\epsilon}\right)^{*}$ with $\gamma^{\epsilon} \subset U$ and that $0 \in Q_{w}$ for $w \in U$. We conclude that $Q_{0}$ contains the open subset $U$ of $\left(M^{\epsilon}\right)^{*}$; consequently, the complex variety $Q_{0}$ is the whole space $\mathbb{C}^{2 n}$, since $M^{\epsilon}$ is generic. Therefore, $r_{j}(\xi, \eta, 0,0) \equiv 0$ for $j=1, \ldots, m$.

Our next step is to find new coordinates for a fixed $M^{\epsilon}$ so that it has two Segre varieties forming part of coordinate subspaces. To this end, we fix a small $\epsilon$ so that $M^{\epsilon}$ contains two Segre varieties $Q_{\gamma^{\epsilon}\left(t^{\prime}\right)}$ and $Q_{\gamma^{\epsilon}\left(t^{\prime \prime}\right)}$ intersecting transversely at the origin. As shown above, $Q_{\gamma^{\epsilon}\left(t^{\prime}\right)}, Q_{\gamma^{\epsilon}\left(t^{\prime \prime}\right)}$ are given by $y^{\prime}=g\left(x, y^{\prime \prime}\right)$ and $y^{\prime}=h\left(x, y^{\prime \prime}\right)$, respectively. Moreover, $g(0)=h(0)=0$. Since $Q_{\gamma\left(t^{\prime}\right)}$ is contained in $M$, then

$$
r_{j}\left(x, g\left(x, y^{\prime \prime}\right), y^{\prime \prime}, \bar{x}, \bar{g}\left(\bar{x}, \bar{y}^{\prime \prime}\right), \bar{y}^{\prime \prime}\right) \equiv 0
$$

for $j=1, \ldots, m$. Hence,

$$
\left[g_{j}\left(x, y^{\prime \prime}\right)\right]_{1} \bar{x}_{\sigma(j)}+{\overline{\left[g_{\sigma(j)}\left(x, y^{\prime \prime}\right)\right.}}_{1} x_{j}=0 .
$$

This means that $\left[g_{1}\right]_{1}, \ldots,\left[g_{m}\right]_{1}$ satisfy $(3.23)$, so $\left[g_{j}\right]_{1}(x, y)=c_{j} x_{j}$. Similarly, $\left[h_{j}\right]_{1}(x, y)=d_{j} x_{j}$. Define the new coordinates

$$
x_{j}^{*}=y_{j}-g_{j}\left(x, y^{\prime \prime}\right), \quad y_{j}^{*}=\left(y_{j}-h_{j}\left(x, y^{\prime \prime}\right)\right) /\left(c_{j}-d_{j}\right), \quad j=1, \ldots, m,
$$

for which the linear terms are of the form (3.25). Now $M \equiv M^{\epsilon}$ contains two Segre varieties $x_{1}^{*}=\cdots=x_{m}^{*}=0$ and $y_{1}^{*}=\cdots=y_{m}^{*}=0$. For brevity, we replace $z^{*}$ by $z$. In view of $r_{j}(z, 0)=r_{j}(0, \bar{z}) \equiv 0$ and the reality condition (3.21), one sees that $M$ is given by (3.27) with

$$
\begin{aligned}
R_{j}(z, \bar{z})= & \sum_{k, l=1}^{m} x_{k} \bar{y}_{l} a_{j k l}^{\prime}(z, \bar{z})+\sum_{k, l=1}^{m} \bar{x}_{k} y_{l} a_{j k l}^{\prime \prime}(\bar{z}, z) \\
& +\sum_{k, l=1}^{m} x_{k} y_{l} b_{j k l}^{\prime}\left(z, \bar{z}^{\prime \prime}\right)+\sum_{k, l=1}^{m} \bar{x}_{k} \bar{y}_{l} b_{j k l}^{\prime \prime}\left(\bar{z}, z^{\prime \prime}\right),
\end{aligned}
$$

where $a_{j k l}^{\prime}(z, \bar{z})$ is independent of $x_{1}, \ldots, x_{k-1}, \bar{y}_{1}, \ldots, \bar{y}_{l-1}$, and $a_{j k l}^{\prime \prime}(\bar{z}, z)$ is independent of $\bar{x}_{1}, \ldots, \bar{x}_{k-1}, y_{1}, \ldots, y_{l-1}$. Obviously $b_{j k l}^{\prime}, b_{j k l}^{\prime \prime}$, independent of $x_{1}, \ldots, x_{k-1}, y_{1}, \ldots, y_{l-1}$, are unique, and $b_{\sigma(j) k l}^{\prime \prime}\left(\bar{z}, z^{\prime \prime}\right)=\bar{b}_{j k l}^{\prime}\left(\bar{z}, z^{\prime \prime}\right)$ by the reality condition $\bar{R}_{j}=R_{\sigma(j)}$. Put $a_{j k l}=\left(a_{j k l}^{\prime}+\bar{a}_{\sigma(j) k l}^{\prime \prime}\right) / 2$. Then the reality condition $\bar{R}_{j}=R_{\sigma(j)}$ implies that $R_{j}$ are given by $(3.27)-(3.29)$. Note that $a_{j k l}(z, \bar{z})$ remains independent of $x_{1}, \ldots, x_{k-1}, \bar{y}_{1}, \ldots, \bar{y}_{l-1}$.

To achieve (3.30), we need to change coordinates one more time. We shall leave the Segre varieties $x_{1}=\cdots=x_{m}=0$ and $y_{1}=\cdots=y_{m}=0$ 
unchanged. Thus, we consider a holomorphic transformation

$$
\psi: x_{j} \rightarrow x_{j}+u_{j}(z), \quad y_{j} \rightarrow y_{j}+v_{j}(z), \quad j=1, \ldots, m
$$

with

$$
\left.u_{j}\right|_{x^{\prime}=0}=0,\left.\quad v_{j}\right|_{y^{\prime}=0}=0, \quad u_{j}, v_{j}=O(2) .
$$

Put $\psi^{-1}(M)$ in the form of (3.27)-(3.29) with $a_{j k l}^{*}$ in place of $a_{j k l}$. Thus, from $r_{j}^{*}=r_{j} \circ \psi$ one obtains

$$
\begin{array}{r}
a_{j}^{*}(z, \bar{z})+\bar{a}_{\sigma(j)}^{*}(\bar{z}, z)=\bar{y}_{\sigma(j)} u_{j}+x_{j} \bar{v}_{\sigma(j)}+y_{j} \bar{u}_{\sigma(j)}+\bar{x}_{\sigma(j)} v_{j} \\
+u_{j} \bar{v}_{\sigma(j)}+\bar{u}_{\sigma(j)} v_{j}+a_{j}\left(z^{*}, \bar{z}^{*}\right)+\bar{a}_{\sigma(j)}\left(\bar{z}^{*}, z^{*}\right)
\end{array}
$$

for $z^{*}=\left(x^{\prime}+u, x^{\prime \prime}, y^{\prime}+v, y^{\prime \prime}\right)$ and

$$
a_{j}(z, \bar{z})=\sum x_{k} \bar{y}_{l} a_{j k l}(z, \bar{z}), \quad a_{j}^{*}(z, \bar{z})=\sum x_{k} \bar{y}_{l} a_{j k l}^{*}(z, \bar{z}) .
$$

Comparing the coefficients of $\bar{y}_{\sigma(j)} x^{\alpha} y^{\beta}$ and $x_{j} \bar{x}^{\alpha} \bar{y}^{\beta}$ in (3.35), respectively, one gets

$$
\begin{gathered}
\sum_{k=1}^{m} x_{k} a_{j k \sigma(j)}^{*}(z, 0)=u_{j}+\sum_{k=1}^{m}\left(x_{k}+u_{k}(z)\right) a_{j k \sigma(j)}(x+u, y+v, 0), \\
\sum_{l=1}^{m} \bar{y}_{l} a_{j j l}^{*}(0, \bar{z})=\bar{v}_{\sigma(j)}+\sum_{l=1}^{m}\left(\bar{y}_{l}+\bar{v}_{l}(\bar{z})\right) a_{j j l}(0, \bar{x}+\bar{u}, \bar{y}+\bar{v}) .
\end{gathered}
$$

Now, the implicit function theorem implies that there exist unique $u, v=$ $O(2)$ satisfying

$$
\sum_{k=1}^{m} x_{k} a_{j k \sigma(j)}^{*}(z, 0)=\sum_{l=1}^{m} \bar{y}_{l} \bar{a}_{j j l}^{*}(0, \bar{z})=0, \quad j=1, \ldots, m .
$$

Obviously, the unique solution $(u, v)$ satisfies (3.34) also. Note that in the decompositions $(3.28), a_{j k \sigma(j)}^{*}(z, 0)$ is independent of $x_{1}, \ldots, x_{k-1}$ and $a_{j j l}^{*}(0, \bar{z})$ is independent of $\bar{y}_{1}, \ldots, \bar{y}_{l-1}$. Now identities (3.36) yield (3.30). This completes the proof of the proposition.

Proof of Theorem 3.6. - Following [6], we want to show that the Levi-flat set $M$, defined by (3.27)-(3.30), is the one given by (3.22).

Consider a real manifold in $M$ parameterized by $x^{\prime}=(1, \ldots, 1)$, $x^{\prime \prime}=y^{\prime \prime}=0$ and

$$
y_{j}(t)= \begin{cases}i t_{j}+\alpha_{j}(t), & \sigma(j)=j \\ t_{j}+i t_{\sigma(j)}+\alpha_{j}(t), & \sigma(j)>j \\ -t_{\sigma(j)}+i t_{j}+\alpha_{j}(t), & \sigma(j)<j\end{cases}
$$

TOME 51 (2001), FASCICULE 1 
with $t \in \mathbb{R}^{m}, \alpha_{j}(0)=0$ and $\alpha_{\sigma(j)}(t)=\bar{\alpha}_{j}(t)$. By the implicit function theorem, such $\alpha_{j}$ exist.

The Segre variety $Q_{\gamma(t)}$ is defined by

$$
y_{j}+\bar{y}_{\sigma(j)}(t) x_{j}+R_{j}(z, \bar{\gamma}(t))=0, \quad j=1, \ldots, m .
$$

By rescaling the coordinates, one may assume that $R_{j}$ are small functions. Hence, $Q_{\gamma(t)}$, intersecting with a fixed neighborhood of the origin, is a smooth complex submanifold given by

$$
y_{j}=h_{j}\left(x, y^{\prime \prime}, t\right), \quad h_{j}\left(x, y^{\prime \prime}, 0\right)=h_{j}(0, t)=0
$$

for $j=1, \ldots, m$, where the last identity comes from $0 \in Q_{\gamma(t)}$.

Since $Q_{\gamma(t)}$ is contained in $M$, we have

$$
\begin{aligned}
0 \equiv & x_{j} \bar{h}_{\sigma(j)}\left(\bar{x}, \bar{y}^{\prime \prime}, t\right)+\bar{x}_{\sigma(j)} h_{j}\left(x, y^{\prime \prime}, t\right) \\
& +\sum\left\{x_{k} \bar{h}_{l}\left(\bar{x}, \bar{y}^{\prime \prime}, t\right) a_{j k l}(z, \bar{z})+\bar{x}_{k} h_{l}\left(x, y^{\prime \prime}, t\right) \bar{a}_{\sigma(j) k l}(\bar{z}, z)\right\} \\
& +\sum\left\{x_{k} h_{l}\left(\bar{x}, \bar{y}^{\prime \prime}, t\right) b_{j k l}\left(z, \bar{z}^{\prime \prime}\right)+\bar{x}_{k} \bar{h}_{l}\left(\bar{x}, \bar{y}^{\prime \prime}, t\right) \bar{b}_{\sigma(j) k l}\left(\bar{z}, z^{\prime \prime}\right)\right\}
\end{aligned}
$$

for $z=\left(x, h\left(x, y^{\prime \prime}\right), y^{\prime \prime}\right)$. Expanding the right-hand side of (3.37) as power series in $x, y^{\prime \prime}, \bar{x}, \bar{y}^{\prime \prime}$ and collecting quadratic terms yields

$$
\left[h_{j}\right]_{1}\left(x, y^{\prime \prime}, t\right)=c_{j}(t) x_{j}, \quad c_{\sigma(j)}(t)+\bar{c}_{j}(t)=0 .
$$

Now looking at (3.37), we see that the homogeneous terms $x^{\alpha} y^{\prime \prime} \bar{x}_{\sigma(j)}$ for $|\alpha|+|\beta|=2$ give us

$$
\begin{aligned}
{\left[h_{j}\right]_{2}\left(x, y^{\prime \prime}, t\right)=} & -\sum x_{k} \bar{c}_{\sigma(j)}(t)\left[a_{j k \sigma(j)}\right]_{1}\left(x,[h]_{1}\left(x, y^{\prime \prime}, t\right), y^{\prime \prime}, 0\right) \\
& -\sum\left[h_{l}\right]_{1}\left(x, y^{\prime \prime}, t\right)\left[\bar{a}_{\sigma(j) \sigma(j) l}\right]_{1}\left(0, x,[h]_{1}\left(x, y^{\prime \prime}, t\right), y^{\prime \prime}\right) .
\end{aligned}
$$

¿From (3.30) it follows that $\left[h_{j}\right]_{2} \equiv 0$. Assuming for the sake of induction that $\left[h_{j}\right]_{2}=\cdots=\left[h_{j}\right]_{N}=0$ for $1 \leq j \leq m$, one gets from (3.30) and (3.37) that

$$
\begin{aligned}
{\left[h_{j}\right]_{N+1}\left(x, y^{\prime \prime}, t\right)=} & \sum x_{k} \bar{c}_{\sigma(j)}(t)\left[a_{j k \sigma(j)}\right]_{N}(x, y, 0) \\
& +\sum\left[h_{l}\right]_{1}\left(x, y^{\prime \prime}, t\right)\left[\bar{a}_{\sigma(j) \sigma(j) l}\right]_{N}(0, x, y) \equiv 0
\end{aligned}
$$

for $y^{\prime}=[h]_{1}\left(x, y^{\prime \prime}, t\right)$. This shows that $h_{j}\left(x, y^{\prime \prime}, t\right)$ are linear in $x$, and that $Q_{\gamma(t)}$ is given by

$$
y_{j}=c_{j}(t) x_{j}, \quad c_{\sigma(j)}(t)+\bar{c}_{j}(t)=0, \quad j=1, \ldots, m .
$$

Therefore, $M$ contains a portion of $\mathcal{Q}$; consequently, $M$ and $\mathcal{Q}$ coincide as they are irreducible. The proof of the theorem is complete. 


\section{Invariants of Levi-flat sets and symplectic transformations.}

Results in the preceding section allow one to choose much simpler defining functions of Levi-flat sets in suitable holomorphic coordinates. In this section we shall use (more restrictive) symplectic coordinates to study the defining functions for the Levi-flat sets that are invariant under a holomorphic symplectic mapping.

This section is divided into two parts: We shall first complete the proofs of Theorem 1.1 and Theorem 1.3 after we obtain the first-integrals from Levi-flat invariant sets. We then turn to a meromorphic eigenfunction problem, arising from Levi-flat sets, and formulate Theorem 4.9, from which Theorem 1.2 follows. The proof of Theorem 4.9 is given in Section 5 .

¿From now on, Levi-flat sets in $\mathbb{C}^{2 n}$ are of codimension $n$. We shall also take into account that in section 3 we ignored the symplectic invariants $\mu_{j}$ and used a simpler permutation $\sigma$ defined by (3.1). Therefore, we need to return to the 4 types of singularities, which involve $\mu_{j}$ (and a more general $\sigma$ in case of (4.21) below).

Let $\sigma$ be a permutation of $1, \ldots, n$ satisfying

$$
\sigma(j)=j, \quad 1 \leq j \leq K ; \quad \sigma(j) \neq j=\sigma^{2}(j), \quad j>K .
$$

We first consider $\mathcal{Q} \subset \mathbb{C}^{2 n}$ defined by

$$
q_{j}(\xi, \eta)+\mu_{j} \bar{q}_{\sigma(j)}(\bar{\xi}, \bar{\eta})=0, \quad j=1, \ldots, n
$$

with

$$
\begin{gathered}
\mu_{j}=1, \quad 1 \leq j \leq K ; \quad \mu_{\sigma(j)}=\bar{\mu}_{j}, \quad\left|\mu_{j}\right|=1, \quad j>K \\
q_{j}(\xi, \eta)= \begin{cases}\sum_{k=1}^{n} a_{j k} \xi_{k} \eta_{k}, & 1 \leq j \leq K, \\
\xi_{j} \eta_{\sigma(j)}, & K<j \leq K+2 L=n\end{cases}
\end{gathered}
$$

with $\left(a_{i j}\right)_{1 \leq i, j \leq K}$ of rank $K$. Note again that we allow $K=0$, or $n$ as special cases.

With the above notations, consider a Levi-flat real analytic set of the form

$$
q_{j}(\xi, \eta)+\mu_{j} \bar{q}_{\sigma(j)}(\bar{\xi}, \bar{\eta})+R_{j}(\xi, \eta, \bar{\xi}, \bar{\eta})=0, \quad j=1, \ldots, n
$$

with

$$
R_{\sigma(j)}(\xi, \eta, \bar{\xi}, \bar{\eta})=\mu_{j} \bar{R}_{j}(\bar{\xi}, \bar{\eta}, \xi, \eta)
$$

TOME 51 (2001), FASCICULE 1 
Let $\xi^{\prime}=\sqrt{\bar{\mu}} \xi \equiv\left(\sqrt{\bar{\mu}_{1}} \xi_{1}, \ldots, \sqrt{\bar{\mu}_{n}} \xi_{n}\right), \eta^{\prime}=\eta$. Then equations (4.4)-(4.5), after multiplied by $\sqrt{\bar{\mu}_{j}}$, are of the form

$$
\tilde{q}_{j}\left(\xi^{\prime}, \eta^{\prime}\right)+\overline{\tilde{q}}_{\sigma(j)}\left(\bar{\xi}^{\prime}, \bar{\eta}^{\prime}\right)+\tilde{R}_{j}\left(\xi^{\prime}, \eta^{\prime}, \bar{\xi}^{\prime}, \bar{\eta}^{\prime}\right)=0
$$

with

$$
\tilde{q}_{j}\left(\xi^{\prime}, \eta^{\prime}\right)=\sqrt{\bar{\mu}_{j}} q_{j}\left(\sqrt{\mu} \xi^{\prime}, \eta^{\prime}\right), \quad \tilde{R}_{j}\left(\xi^{\prime}, \eta^{\prime}, \bar{\xi}^{\prime}, \bar{\eta}^{\prime}\right)=\sqrt{\bar{\mu}_{j}} R_{j}\left(\sqrt{\mu} \xi^{\prime}, \eta^{\prime}\right) .
$$

Now Theorem 3.1 says that the set is actually given by

$$
\tilde{q}_{j}\left(\xi^{\prime}, \eta^{\prime}\right)+\overline{\tilde{q}}_{\sigma(j)}\left(\bar{\xi}^{\prime}, \bar{\eta}^{\prime}\right)+\tilde{H}_{j}\left(\xi^{\prime}, \eta^{\prime}\right)+\bar{H}_{\sigma(j)}\left(\bar{\xi}^{\prime}, \bar{\eta}^{\prime}\right)=0
$$

Returning to the original coordinates and putting

$$
H_{j}(\xi, \eta)=\sqrt{\mu_{j}} \tilde{H}_{j}(\sqrt{\bar{\mu}} \xi, \eta)
$$

one sees that the set is of the form

$$
M: r_{j}=q_{j}(\xi, \eta)+\mu_{j} \bar{q}_{\sigma(j)}(\bar{\xi}, \bar{\eta})+H_{j}(\xi, \eta)+\mu_{j} \bar{H}_{\sigma(j)}(\bar{\xi}, \bar{\eta})=0
$$

for $j=1, \ldots, n$, where $\sigma, q_{j}$, and $\mu_{j}$ are given by (4.1) and (4.3), and $H_{j}(\xi, \eta)=O(3)$ are holomorphic.

Theorem 4.1. - Let $M, H_{j}, q_{j}$ be as in (4.6). Let $\varphi$ be a holomorphic symplectic mapping defined by (1.1)-(1.2) with $\varphi(M)=M$, and $\Phi$ a formal symplectic transformation such that $\Phi \varphi \Phi^{-1}$ is in the Birkhoff normal form (1.3). Then $\left|\lambda_{\sigma(j)}\right|=\left|\lambda_{j}\right|$ and

$$
\left(q_{j}+H_{j}\right) \circ \Phi^{-1}(\xi, \eta)=q_{j}(\xi, \eta)+\widehat{H}_{j}\left(\xi \eta, \xi^{\prime \prime} \eta_{\sigma}^{\prime \prime}\right), \quad j=1, \ldots, n
$$

with $\widehat{H}_{j}\left(\xi \eta, \xi^{\prime \prime} \eta_{\sigma}^{\prime \prime}\right)=O\left(|(\xi, \eta)|^{4}\right)$ and $\xi^{\prime \prime} \eta_{\sigma}^{\prime \prime}=\left(\xi_{K+1} \eta_{\sigma(K+1)}, \ldots, \xi_{m} \eta_{\sigma(m)}\right)$.

Anticipating the proof of Theorem 4.1, we first prove Theorem 1.1 and Theorem 1.3.

Proof of Theorem 1.1. — Let $M$ be the Levi-flat real analytic set defined by

$$
\Re\left\{\sum_{k=1}^{n} a_{j k} \xi_{k} \eta_{k}\right\}+R_{j}(\xi, \eta, \bar{\xi}, \bar{\eta})=0, \quad j=1, \ldots, n,
$$

where the matrix $\left(a_{j k}\right)$ is of rank $n$ and $R_{j}(\xi, \eta, \bar{\xi}, \bar{\eta})=O(3)$ are real-valued convergent power series. In Section 3 (Theorem 3.1) we have simplified the equation of $M$, (from now on) given by

$$
\Re\left\{q_{j}(\xi, \eta)+H_{j}(\xi, \eta)\right\}=0, \quad j=1, \ldots, n
$$

with $H_{j}(\xi, \eta)=O(3)$ holomorphic, and $q_{j}=\sum_{k=1}^{n} a_{j k} \xi_{k} \eta_{k}$. 
Theorem 4.1 establishes that if a symplectic mapping $\varphi$, given by (1.1)-(1.2) leaves the set $M$ invariant, and if $\Phi$ is a formal symplectic transformation that puts $\varphi$ into its Birkhoff normal form, then $\left(q_{j}+H_{j}\right) \circ$ $\Phi^{-1}$ are power series which depend only on the products $\xi_{j} \eta_{j}$. (Indeed under our present hypotheses $q_{j}$ depend only on $\xi \eta$ and $\sigma$ is just the identity.) These power series are thus invariant under the action of the formally normalized mapping. Hence the functions $q_{j}+H_{j}$ are invariant, under the action of $\varphi$. Since $\left(a_{j k}\right)_{1 \leq j, k \leq n}$ is nonsingular, a theorem of Vey [18] says that $\varphi$ is normalizable by convergent transformations. The proof of Theorem 1.1 is complete.

Proof of Theorem 1.3. - Let $r$ be the defining function of $M$, and $q$ the quadratic form of $r$. Note that the order of $r$ might be one, which can however be ruled out by applying the argument below to $r^{2}$.

Since $M$ is Levi-flat and $\mathcal{Q}: q=0$ is a hypersurface in $\mathbb{C}^{2}$, then $\mathcal{Q}$ is also Levi-flat [6]. Next, we want to show that $\mathcal{Q}$ is invariant under $\Lambda$. Consider first the case that $M$ is the union of two distinct smooth real hypersurfaces. In this case, $r=r_{1} r_{2}$ with $r_{1}(0)=r_{2}(0)=0$. Since $r \circ \varphi$ vanishes on $r_{1}=0$ and on $r_{2}=0$, then $r_{1}, r_{2}$ divide $r \circ \varphi$. In particular, $r \circ \varphi=u r$. Thus, $q \circ \Lambda=u(0) q$, i.e., $\Lambda(\mathcal{Q})=\mathcal{Q}$. Next, we assume that $M$ is irreducible. Then either $r$ is irreducible, or $r=u r_{1}^{2}$ with $r_{1}(0)=0$. When $r$ is irreducible, $r(\xi, \eta, \bar{\xi}, \eta)$ is also irreducible as a germ of holomorphic function in $\xi, \eta, \bar{\xi}, \bar{\eta}$, because $M$ is of codimension 1 (e.g., see [6]). Since $r \circ \varphi$ vanishes on $M^{c}$, then $r$ divides $r \circ \varphi$, which implies that $\Lambda(\mathcal{Q})=\mathcal{Q}$. When $r=u r_{1}^{2}$, it is clear that $r_{1}$ divides $r_{1} \circ \varphi$. Denote by $a \xi+b \eta$ the linear part of $r_{1}$. Then $a \lambda \xi+b \bar{\lambda} \eta=u(0)(a \xi+b \eta)$. Since $u(0)$ is real, the above identity contradicts that not all $a, b$ are zero. (Note that the contradiction also implies that $r$ starts with quadratic terms, as claimed at the beginning of the proof.) Therefore, $\mathcal{Q}$ is invariant under $\Lambda$.

Applying the above argument to $\mathcal{Q}$, one sees that all germs of real analytic functions vanishing on $\mathcal{Q}$ are divisible by $q$, i.e., that $\mathcal{I}=\mathcal{I}_{\mathcal{Q}}$ is spanned by the real quadratic form $q$. Hence, $\mathcal{I}$ is contained in one of $E_{i j}$ and $E_{1}$. Since the former contain no $\Lambda$-invariant $\mathbb{C}$-linear subspace of dimension one, then $\mathcal{I}$ is contained in $E_{1}$. Hence, we get $q(\xi, \eta, \bar{\xi}, \bar{\eta})=$ $\Re\{a \xi \eta\}+b|\xi|^{2}+c|\eta|^{2}$. If $b=c=0$, Theorem 1.1 says that $\varphi$ is normalizable by holomorphic symplectic transformations.

If $b$ or $c$ is not zero, a result in [6] says that $a=0$. Thus, we see that 
$M$ is given by

$$
b|\xi|^{2}+c|\eta|^{2}+R(\xi, \eta, \bar{\xi}, \bar{\eta})=0, \quad b \cdot c<0, \quad b, c \in \mathbb{R}
$$

with $R(\xi, \eta, \bar{\xi}, \bar{\eta})=O(3)$ real-valued. By a change of symplectic coordinates, one may assume $b=-c$. Of course the proof of the theorem is complete, by applying Theorem 1.2.

Alternatively, one can prove the theorem without using Theorem 1.2 when $M$ is given by (4.7), for which we shall prove the next result.

Proposition 4.2. - Let $\varphi$ be a holomorphic symplectic mapping of $\mathbb{C}^{2}$ of the form

$$
\xi^{\prime}=\lambda \xi+O(2), \quad \eta^{\prime}=\lambda^{-1} \eta+O(2), \quad \lambda^{2} \neq 1 .
$$

Let $M \subset \mathbb{C}^{2}$ be a Levi-flat real analytic hypersurface given by (4.7). Assume that as germs of real analytic sets, $\varphi(M)=M$. Then $|\lambda|=1$, and there exists a single change of holomorphic symplectic coordinates that puts $M$ into the complex cone $|\xi|^{2}-|\eta|^{2}=0$ and linearizes $\varphi$ simultaneously.

Proof. - Notice that for this proposition, we allow $\lambda$ to be a root of unity. Also, it is obvious that $|\lambda|=1$, since $r(\xi, \eta, \bar{\xi}, \bar{\eta})=b|\xi|^{2}+c|\eta|^{2}+$ $R(\xi, \eta, \bar{\xi}, \bar{\eta})$ is irreducible and $r \circ \varphi=d r$ for some analytic real function $d$.

Since $M$ is Levi-flat, a theorem in [6] says that there is a holomorphic transformation $\psi_{1}$ such that $\psi_{1}(M)$ is the complex cone $|\xi|^{2}-|\eta|^{2}=0$. (See also Theorem 3.6 in Section 3.) Next, we want to find a holomorphic mapping

$$
\psi_{2}: \xi^{\prime}=p(\xi, \eta) \xi, \quad \eta^{\prime}=p(\xi, \eta) \eta
$$

such that the Jacobian determinant of $\psi_{2}$ satisfies

$$
\operatorname{det}\left(D \psi_{2}\right)=\left\{\operatorname{det}\left(D \psi_{1}\right)\right\} \circ\left\{\psi_{1}^{-1}\right\} .
$$

Thus, $\psi=\psi_{2} \circ \psi_{1}$ is symplectic, and $\psi(M)$ is still the cone $|\xi|^{2}-|\eta|^{2}=0$. Write the right-hand side of $(4.8)$ as $a_{0}^{2}(1+u(\xi, \eta))$ with $a_{0} \neq 0$ a constant, and $u(0)=0$. We seek a solution of the form $p(\xi, \eta)=a_{0}(1+v(\xi, \eta))$. Thus, (4.8) becomes

$$
\xi v_{\xi}+\eta v_{\eta}+2 v=\left(\xi v_{\xi}+v\right)\left(\eta v_{\eta}+v\right)-\xi v_{\xi} \cdot \eta v_{\eta}+u
$$

Comparing coefficients, one sees that there exists a unique formal power series solution $v(\xi, \eta)$ with $v(0)=0$. Let $u_{i j}, v_{i j}$ be the coefficients of $u, v$, respectively. Put

$$
u^{*}=\sum\left|u_{i j}\right| \xi^{i} \eta^{j}, \quad w=\sum(i+j+2)\left|v_{i j}\right| \xi^{i} \eta^{j} .
$$


Obviously, $\xi v_{\xi}, \eta v_{\eta}, \xi v_{\xi}+v, \eta v_{\eta}+v$ are majorized by $w$, for which we write $\xi v_{\xi} \prec w$, etc. Thus, (4.9) implies that

$$
w \prec 2 w^{2}+u^{*}, \quad w(0)=u^{*}(0)=0 .
$$

By the Cauchy majorant argument, $w$, and hence $v$, is convergent.

Now $\varphi^{*}=\psi \varphi \psi^{-1}$ is a holomorphic mapping sending the complex cone $|\xi|^{2}-|\eta|^{2}=0$ into itself. In particular, $\varphi^{*}$ sends a Segre variety of the cone into another Segre variety, i.e., $\varphi^{*}$ sends a complex line (inside the cone) through the origin to another complex line. Hence, one first sees that $\varphi^{*}$ leaves the eigenspaces of its linear part invariant. Thus, $\lambda \neq \bar{\lambda}$ implies that

$$
\varphi^{*}: \xi \rightarrow f(\xi, \eta) \lambda \xi, \quad \eta \rightarrow g(\xi, \eta) \bar{\lambda} \eta
$$

with $f(0)=g(0)=1$. Furthermore, $g / f$ is holomorphic near the origin, and remains constant on each complex line in the cone. Therefore, $f \equiv g$ on the cone, and hence near the origin in $\mathbb{C}^{2}$. This shows that $\varphi^{*}$ is of the form $(\xi, \eta) \rightarrow f(\xi, \eta)(\lambda \xi, \bar{\lambda} \eta)$ with $f(0)=1$. Since $\operatorname{det}\left(\partial \varphi^{*} / \partial(\xi, \eta)\right)=1$, we obtain $f(\xi, \eta) \equiv 1$. In particular, $\psi \circ \varphi \circ \psi^{-1}=\varphi^{*}$ is a linear transformation.

We now turn to the proof Theorem 4.1. Let us first prove the following.

Lemma 4.3. - Let $\mathcal{Q} \subset \mathbb{C}^{2 n}$ be defined by (4.2)-(4.3), and let $h$ be a holomorphic homogeneous polynomial in $\xi, \eta$. Assume that $h$ is realvalued on $\mathcal{Q}$. Then $h$ is a polynomial in $q_{1}, \ldots, q_{n}$.

Proof. - By changing linear coordinates, we may assume that $\mu_{j}=$ -1 , and that $\sigma(j)=L+j$ for $j=K+1, \ldots, K+L$. Put $\xi=\left(\xi^{\prime}, \xi^{\prime \prime}\right), \eta=$ $\left(\eta^{\prime}, \eta^{\prime \prime}\right)$ with

$$
\begin{aligned}
\xi^{\prime \prime} & =\left(\xi_{K+1}, \ldots \xi_{n}\right)=\left(x_{K+1}, \ldots, x_{K+L}, y_{K+1}, \ldots, y_{K+L}\right) \\
\eta^{\prime \prime} & =\left(\eta_{K+1}, \ldots \eta_{n}\right)=\left(z_{K+1}, \ldots, z_{K+L}, w_{K+1}, \ldots, w_{K+L}\right)
\end{aligned}
$$

Assume first that $h$ depends only on $\xi^{\prime \prime}, \eta^{\prime \prime}$. Expand $h(x, y, z, w)=$ $\sum h_{a b c d} x^{a} y^{b} z^{c} w^{d}$. On $\mathcal{Q}$, one has $w_{j}=\bar{y}_{j} \bar{z}_{j} x_{j}^{-1}$ for $j=K+1, \ldots, K+L$. Since $h$ is real on $\mathcal{Q}$, then

$$
\sum h_{a b c d} x^{a-d} y^{b} z^{c} \bar{y}^{d} \bar{z}^{d}=\sum \bar{h}_{a b c d} y^{d} z^{d} \bar{x}^{a-d} \bar{y}^{b} \bar{z}^{c} .
$$

Fix $a, b, c, d$ with $h_{a b c d} \neq 0$. Comparing two sides of the above identity, one sees that there exist $a^{\prime}, b^{\prime}, c^{\prime}, d^{\prime}$ such that

$$
(a-d, b, c, 0, d, d)=\left(0, d^{\prime}, d^{\prime}, a^{\prime}-d^{\prime}, b^{\prime}, c^{\prime}\right) .
$$


Hence, $a=d$ and $b=c$. This shows that $h\left(\xi^{\prime \prime}, \eta^{\prime \prime}\right)=\sum h_{a b b a}\left(\xi^{\prime \prime} \eta_{\sigma}^{\prime \prime}\right)^{(a, b)}$.

For the general case, we shall prove by induction on $l$ that there exist decompositions

$$
h(\xi, \eta)=\sum_{|\beta|<l} c_{\alpha \beta}\left(\xi^{\prime \prime}, \eta^{\prime \prime}\right) \xi^{\prime \alpha} \eta^{\beta}+\sum_{|\gamma| \geq l} c_{\gamma}\left(\xi^{\prime \prime} \eta_{\sigma}^{\prime \prime}\right) q_{1}^{\gamma_{1}} \cdots q_{K}^{\gamma_{K}}
$$

for $l=0,1, \ldots$. Fix $\xi^{\prime \prime}, \eta^{\prime \prime}$ satisfying equations (4.2) for $j>K$, and let $\xi^{\prime}, \eta^{\prime}$ satisfy

$$
\sum_{k=1}^{n} a_{j k} \xi_{k} \eta_{k}=t_{j}, \quad j=1, \ldots, K
$$

for $t_{j} \in \mathbb{R}$. Solving for $\eta_{1}, \ldots, \eta_{K}$ yields

$$
\eta_{j}=\xi_{j}^{-1} b_{j}(t)+\xi_{j}^{-1} \sum_{k=K+1}^{n} c_{j k} \xi_{k} \eta_{k}, \quad j=1, \ldots, K,
$$

where $t \rightarrow b(t)=\left(b_{1}(t), \ldots, b_{K}(t)\right)$ is a nonsingular linear transformation. The decompositions (4.10) are trivial for $l>\operatorname{deg} h$. Assuming that the decompositions (4.10) hold for $l>k$, we want to show that (4.10) for $l=k$ is valid. ¿From (4.10)-(4.11) one sees that terms of order $k$ in $t$ are given by

$$
\sum_{|\beta|=k} c_{\alpha \beta}\left(\xi^{\prime \prime}, \eta^{\prime \prime}\right) \xi^{\prime \alpha-\beta} b^{\beta}(t)
$$

For fixed $\xi^{\prime \prime}, \eta^{\prime \prime}$, the above summation is real-valued for $t \in \mathbb{R}^{K}$ and $\xi^{\prime} \in \mathbb{C}^{K}$. Since the transformation $t \rightarrow b(t)$ is nonsingular, we know that on $\mathcal{Q}$

$$
\begin{aligned}
c_{\alpha \beta}\left(\xi^{\prime \prime}, \eta^{\prime \prime}\right)=0, & \alpha \neq \beta,|\beta|=k, \\
\Im\left\{\sum_{|\beta|=k} B_{\alpha}^{\beta} c_{\beta \beta}\left(\xi^{\prime \prime}, \eta^{\prime \prime}\right)\right\} & =0, \quad|\alpha|=k,
\end{aligned}
$$

where $B_{\alpha}^{\beta}$ are the coefficients of the linear transformation

$$
t^{\beta} \rightarrow b^{\beta}(t)=\sum_{|\alpha|=k} B_{\alpha}^{\beta} t^{\alpha}, \quad|\beta|=k .
$$

Now (4.12) implies that for $\alpha$ with $|\alpha|=k, \sum_{|\beta|=k} B_{\alpha}^{\beta} c_{\beta \beta}\left(\xi^{\prime \prime}, \eta^{\prime \prime}\right)$ are polynomials in $\xi^{\prime \prime} \eta_{\sigma}^{\prime \prime}$. Since the linear transformation (4.13) is nonsingular, then $c_{\beta \beta}\left(\xi^{\prime \prime}, \eta^{\prime \prime}\right)$ are power series in $\xi^{\prime \prime} \eta_{\sigma}^{\prime \prime}$ for $|\beta|=k$. Rewrite (4.10) as

$$
h(\xi, \eta)=\sum_{|\beta|<k} \tilde{c}_{\alpha \beta}\left(\xi^{\prime \prime}, \eta^{\prime \prime}\right) \xi^{\prime \alpha} \eta^{\prime \beta}+\sum_{|\gamma| \geq k} c_{\gamma}\left(\xi^{\prime \prime} \eta_{\sigma}^{\prime \prime}\right) q_{1}^{\gamma_{1}} \cdots q_{K}^{\gamma_{K}} .
$$

By induction, the proof of the lemma is complete. 
To state the next result, we need some notation. For a power series $f(\xi, \eta, \bar{\xi}, \bar{\eta})$, denote by $P_{0} f$ the sum of terms in the power series expansion of $f$, which are not of the form $(\xi \eta)^{a}(\bar{\xi} \bar{\eta})^{b}\left(\xi^{\prime \prime} \eta_{\sigma}^{\prime \prime}\right)^{c}\left(\overline{\xi^{\prime \prime}} \eta_{\sigma}^{\prime \prime}\right)^{d}$.

Lemma 4.4. - Let $r_{1}, \ldots, r_{n}$ be the formal power series in (4.6) with $q_{j}$ being (4.3). Let $d_{1}, \ldots, d_{n}$ be power series in $\xi, \eta, \bar{\xi}, \bar{\eta}$ of order at least $k$. Assume that

$$
\left[P_{0} d_{1}\right]_{k} q_{1}+\cdots+\left[P_{0} d_{n}\right]_{k} q_{n}=0 .
$$

Then there exist formal power series $\tilde{d}_{1}, \ldots, \tilde{d}_{n}$ such that

$$
d_{1} r_{1}+\cdots+d_{n} r_{n}=\tilde{d}_{1} r_{1}+\cdots+\tilde{d}_{n} r_{n}
$$

with ord $\tilde{d}_{j} \geq k$ and $\left[P_{0} \tilde{d}_{j}\right]_{k}=0$.

Proof. - Choose $\mathbb{C}$-linear combinations $r_{1}^{*}, \ldots, r_{K}^{*}$ of $r_{1}, \ldots, r_{K}$ so that

$$
r_{j}^{*}(\xi, \eta, \bar{\xi}, \bar{\eta})=\xi_{j} \eta_{j}+\sum_{k>K} b_{j k} \xi_{k} \eta_{k}+\sum_{k=1}^{n} c_{j k} \overline{\xi_{k} \eta_{k}}+O(3) .
$$

It suffices to prove the lemma for

$$
r_{1}^{*}, \ldots, r_{K}^{*}, r_{K+1}, \ldots, r_{n} .
$$

For brevity, we drop the superscript of $r_{j}^{*}$. For $j>1$, decompose $\left[d_{j}\right]_{k}=$ $u_{j} q_{1}+\left[u_{j}^{\prime}\right]_{k}$ so that $\left[u_{j}^{\prime}\right]_{k}$ contains only terms $\xi^{\alpha} \eta^{\beta} \bar{\xi}^{\gamma} \bar{\eta}^{\delta}$ with $\alpha_{1}=0$, or $\beta_{1}=0$. Put

$$
u_{j}^{\prime}=d_{j}-u_{j} r_{1}, \quad j>1 .
$$

Then

$$
d_{1} r_{1}+\cdots+d_{n} r_{n}=\tilde{d}_{1} r_{1}+u_{2}^{\prime} r_{2}+\cdots+u_{n}^{\prime} r_{n} .
$$

Comparing terms $\xi^{\alpha} \eta^{\beta} \bar{\xi}^{\gamma} \bar{\eta}^{\delta}$ with $\alpha_{1}, \beta_{1} \geq 1$, one sees that $\left[P_{0} \tilde{d}_{1}\right]_{k}=0$, and hence

$$
\left[P_{0} u_{2}^{\prime}\right]_{k} q_{2}+\cdots+\left[P_{0} u_{n}^{\prime}\right]_{k} q_{n}=0 .
$$

Inductively, one determines $\tilde{d}_{2}, \ldots \tilde{d}_{K}$ so that

$$
d_{1} r_{1}+\cdots+d_{n} r_{n}=\tilde{d}_{1} r_{1}+\cdots+\tilde{d}_{K} r_{K}+u_{k+1}^{\prime \prime} r_{K+1}+\cdots+u_{n}^{\prime \prime} r_{n}
$$

and $\left[P_{0} \tilde{d}_{1}\right]_{k}=\cdots=\left[P_{0} \tilde{d}_{K}\right]_{k}=0$. To find $\tilde{d}_{K+1}$, we decompose $u_{j}^{\prime \prime}=$ $v_{j} r_{K+1}+v_{j}^{\prime}$ for $j>K+1$ so that $\left[v_{j}^{\prime}\right]_{k}$ contains only terms of the form $\xi^{\alpha} \eta^{\beta} \bar{\xi}^{\gamma} \bar{\eta}^{\delta}$ with $\alpha_{K+1}=0$ or $\beta_{\sigma(K+1)}=0$. Thus,

$$
u_{K+1}^{\prime \prime} r_{K+1}+\cdots+u_{n}^{\prime \prime} r_{n}=\tilde{d}_{K+1} r_{K+1}+v_{K+2}^{\prime} r_{K+2}+\cdots+v_{n}^{\prime} r_{n} .
$$


Comparing the terms of the form $\xi^{\alpha} \eta^{\beta} \bar{\xi}^{\gamma} \bar{\eta}^{\delta}$ with $\alpha_{K+1}, \beta_{\sigma(K+1)} \geq 1$ yields $\left[P_{0} \tilde{d}_{k+1}\right]_{k}=0$. Now the proof of the lemma is complete by induction.

Proof of Theorem 4.1. - By Proposition 3.5, $r_{1} \circ \varphi, \ldots, r_{n} \circ \varphi$ are in the ideal generated by $r_{1}, \ldots, r_{n}$. In particular,

$$
q_{j}\left(\lambda \xi, \lambda^{-1} \eta\right)+\mu_{j} \bar{q}_{\sigma(j)}\left(\bar{\lambda} \xi, \bar{\lambda}^{-1} \eta\right)=\sum_{k=1}^{n} c_{j k}\left(q_{k}(\xi, \eta)+\mu_{k} q_{\sigma(k)}(\bar{\xi}, \bar{\eta})\right.
$$

for some constants $c_{j k}$, where

$$
\lambda \xi \equiv\left(\lambda_{1} \xi_{1}, \ldots, \lambda_{n} \xi_{n}\right), \quad \lambda^{-1} \eta \equiv\left(\lambda_{1}^{-1} \eta_{1}, \ldots, \lambda_{n}^{-1} \eta_{n}\right) .
$$

Since $\left(a_{j k}\right)_{1 \leq j, k \leq K}$ is nonsingular, then $q_{1}, \ldots, q_{n}$ are linearly independent. Now, a simple computation shows that $c_{j j}=1$ for $j=1, \ldots, K$,

$$
\lambda_{j} \lambda_{\sigma(j)}^{-1}=c_{j j}=\bar{\lambda}_{\sigma(j)} \bar{\lambda}_{j}^{-1}
$$

for $j=K+1, \ldots, n$, and $c_{j k}=0$ for $j \neq k$. In particular, $\left|\lambda_{\sigma(j)}\right|=\left|\lambda_{j}\right|$. Now, we have decompositions

$$
\Delta r_{j} \equiv r_{j} \circ \varphi-\lambda_{j} \lambda_{\sigma(j)}^{-1} r_{j}=\sum D_{j k} r_{k}, \quad 1 \leq j \leq m
$$

with

$$
\min _{k}\left\{\operatorname{ord} D_{j k}\right\}=\min \left\{\operatorname{ord} \Delta r_{j}\right\}-2>0 .
$$

By abuse of notation, we replace $\Phi \varphi \Phi^{-1}$ by $\varphi$, and $\Phi(M)$ by $M$, etc. Then $M$ is of the form (4.6), while $H_{1}, \ldots, H_{n}$ are formal power series.

We need to show that

$$
\left[P_{0} H_{j}\right]_{d+1}=0, \quad j=1, \ldots, n .
$$

For the purpose of induction we shall also prove that there exist decompositions

$$
r_{j} \circ \varphi-\lambda_{j} \lambda_{\sigma(j)}^{-1} r_{j}=\sum \widetilde{D}_{j k} r_{k}, \quad j=1, \ldots, n
$$

with

$$
\left[P_{0} \widetilde{D}_{i j}\right]_{d-1}=0 .
$$

Obviously, (4.17)-(4.19) hold for $d=1$ when formulae (4.18) are replaced by (4.15). Assume for induction that $\left[\widetilde{D}_{i j}\right]_{k}$ for $k<d$ have been determined such that (4.18)-(4.19) hold. We would like to show that (4.18)-(4.19) hold if $d$ is replaced by $d+1$. For brevity we shall temporarily replace $\left[H_{j}\right]_{d+2}$ by $H_{j}$, and $\left[D_{j k}\right]_{d}$ by $D_{j k}$, etc. Then by (4.17)-(4.19), terms of order $d+2$ in (4.18) yield

$$
P_{0} K_{j}(\xi, \eta)+\mu_{j} P_{0} \bar{K}_{\sigma(j)}(\bar{\xi}, \bar{\eta})=\sum P_{0} D_{j k} \cdot\left[r_{k}\right]_{2}
$$


with

$$
K_{j}(\xi, \eta)=H_{j}\left(\lambda \xi, \lambda^{-1} \eta\right)-\lambda_{j} \lambda_{\sigma(j)}^{-1} H_{j}(\xi, \eta) .
$$

Obviously, the real and imaginary parts of the left-hand side of (4.20), i.e., $\Re\left\{P_{0} K_{j}+\bar{\mu}_{j} P_{0} K_{\sigma(j)}\right\}$ and $\Re\left\{-i P_{0} K_{j}+i \bar{\mu}_{j} K_{\sigma(j)}\right\}$, vanish on $\mathcal{Q}$. ¿From Lemma 4.3, it follows that $K_{j}$ are polynomials in $q_{1}, \ldots, q_{n}$; in particular, $P_{0} K_{j}=0$. Now the non-resonance condition (1.2) yields $P_{0} H_{j}=0$, which gives us (4.17) with $d+1$ in place of $d$.

We now have

$$
\sum P_{0}\left[\widetilde{D}_{j k}\right]_{d} \cdot\left[r_{k}\right]_{2}=0
$$

In view of Lemma 4.4 , one can modify $\left[\widetilde{D}_{i j}\right]_{d}$ so that (4.18)-(4.19) hold when $d$ is replaced by $d+1$. This completes the proof of the theorem.

The rest of section is to study the Levi-flat real analytic sets $M \subset \mathbb{C}^{2 n}$ of the form

$$
q_{j}(\xi, \eta, \bar{\xi}, \bar{\eta})+R_{j}(\xi, \eta, \bar{\xi}, \bar{\eta})=0, \quad j=1, \ldots, n
$$

with

$$
q_{j}= \begin{cases}\left|\xi_{j}\right|^{2}-\mu_{j}\left|\eta_{\sigma(j)}\right|^{2}, & 1 \leq j \leq K \\ \xi_{j} \bar{\xi}_{\sigma(j)}-\mu_{j} \bar{\eta}_{j} \eta_{\sigma(j)}, & K<j \leq n,\end{cases}
$$

where $\mu_{j}$ and $\sigma$ (a permutation of $1, \ldots, n$ ) satisfy

$$
\begin{array}{r}
1 \leq \sigma(j) \leq K, \quad \mu_{\sigma(j)}=1 / \mu_{j}>0, \quad 1 \leq j \leq K ; \\
\sigma(j)=\sigma^{-1}(j) \neq j, \quad\left|\mu_{j}\right|=1, \quad \mu_{\sigma(j)}=\bar{\mu}_{j}, \quad K<j \leq n
\end{array}
$$

for some $0 \leq K \leq n+1$. We further assume that $R_{j}$ satisfy the reality conditions

$$
\begin{gathered}
R_{j}(\xi, \eta, \bar{\xi}, \bar{\eta})=\bar{R}_{j}(\bar{\xi}, \bar{\eta}, \xi, \eta), \quad 1 \leq j \leq K \\
R_{\sigma(j)}(\xi, \eta, \bar{\xi}, \bar{\eta})=\bar{R}_{j}(\bar{\xi}, \bar{\eta}, \xi, \eta), \quad K<j \leq n
\end{gathered}
$$

With the above assumptions and the change of coordinates $\xi_{j} \rightarrow \xi_{j}, \eta_{j} \rightarrow$ $\sqrt{\mu_{\sigma^{-1}(j)}} \eta_{j}$, we can then apply Theorem 3.6. Thus, $M$ is given by

$$
0=r_{j}= \begin{cases}F_{j}(\xi, \eta) \bar{F}_{j}(\bar{\xi}, \bar{\eta})-\mu_{j} G_{\sigma(j)}(\xi, \eta) \bar{G}_{\sigma(j)}(\bar{\xi}, \bar{\eta}), & j \leq K \\ F_{j}(\xi, \eta) \bar{F}_{\sigma(j)}(\bar{\xi}, \bar{\eta})-\mu_{j} G_{\sigma(j)}(\xi, \eta) \bar{G}_{j}(\bar{\xi}, \bar{\eta}), & j>K\end{cases}
$$

where $\sigma, \mu_{j}$ are given by (4.22), and

$$
\begin{array}{ll}
F_{j}(\xi, \eta)=\xi_{j}+f_{j}(\xi, \eta), & G_{j}(\xi, \eta)=\eta_{j}+g_{j}(\xi, \eta), \\
f_{j}(\xi, \eta)=O(2), & g_{j}(\xi, \eta)=O(2) .
\end{array}
$$

Introduce meromorphic functions

$$
m_{j}(\xi, \eta)=\frac{\xi_{j}+f_{j}(\xi, \eta)}{\eta_{\sigma(j)}+g_{\sigma(j)}(\xi, \eta)}, \quad j=1, \ldots, n
$$

TOME 51 (2001), FASCICULE 1 
Proposition 4.5. - Let $M$ be a real analytic set given by (4.22)(4.24). Then $m_{1}, \ldots, m_{n}$ depend only on $M$. If $M$ is invariant under $\varphi$ defined by (1.1)-(1.2), then $\left|\lambda_{\sigma(j)}\right|=1 /\left|\lambda_{j}\right|$ and

$$
m_{j} \circ \varphi=\lambda_{j} \lambda_{\sigma(j)} m_{j}, \quad j=1, \ldots, n .
$$

Proof. - We first want to show that $\mu_{j}, m_{j}$ are uniquely determined by $M$. Assume that $M$ is also given by $(4.23)-(4.24)$ with $r_{j}^{*}, \mu_{j}^{*}, f_{j}^{*}, g_{j}^{*}$ in place of $r_{j}, \mu_{j}, f_{j}, g_{j}$, respectively. Define $m_{j}^{*}$ as in (4.25). By the Weierstrass preparation theorem, we may assume that the coefficients of $g_{j}, g_{j}^{*}$ satisfy the normalizing condition

$$
g_{j \alpha \beta}=0, \quad \beta_{j} \neq 0 .
$$

We need to show that $\mu_{j}^{*}=\mu_{j}, f_{j}^{*}=f_{j}$, and $g_{j}^{*}=g_{j}$. Since $r_{j}^{*}-r_{j}$ vanishes on $M$, it follows form Proposition 3.7 that

$$
r_{j}^{*}-r_{j}=\sum D_{j k} r_{k}
$$

with

$$
\min \left\{\operatorname{ord} D_{j k}\right\}=\operatorname{ord}\left(r_{j}^{*}-r_{j}\right)-2 .
$$

Comparing the quadratic terms in (4.28) gives $\mu_{j}^{*}=\mu_{j}$ and $D_{j k}(0)=0$.

Assume for induction that

$$
\Delta f_{j} \equiv f_{j}^{*}-f_{j}=O(d), \quad \Delta g_{j} \equiv g_{j}^{*}-g_{j}=O(d), \quad D_{j k}=O(d-1) .
$$

For brevity, we replace $\left[f_{j}\right]_{d}$ by $f_{j}$, and $\left[D_{j k}\right]_{d-1}$ by $D_{j k}$, etc. In (4.28) terms of the form $\xi^{\alpha} \eta^{\beta} \bar{\xi}^{\gamma} \bar{\eta}^{\delta}$ with $|\alpha|+|\beta|=d$ and $|\gamma|+|\delta|=1$ give us

$$
\begin{aligned}
& \bar{\xi}_{j} \Delta f_{j}-\mu_{j} \bar{\eta}_{\sigma(j)} \Delta g_{\sigma(j)}=\sum D_{j k}(\xi, \eta, 0,0)\left[r_{k}\right]_{2}, \quad j \leq K, \\
& \bar{\xi}_{\sigma(j)} \Delta f_{j}-\mu_{j} \bar{\eta}_{j} \Delta g_{\sigma(j)}=\sum D_{j k}(\xi, \eta, 0,0)\left[r_{k}\right]_{2}, \quad j>K .
\end{aligned}
$$

On $\mathcal{Q}$, the right-hand sides of (4.30)-(4.31) vanish and one can put

$$
\begin{array}{lll}
\eta_{\sigma(j)}=\rho_{j} \bar{\xi}_{j} / \sqrt{\mu_{j}}, & \left|\rho_{j}\right|=1, & j \leq K \\
\eta_{j}=\sqrt{\mu_{j}} \rho_{j} \bar{\xi}_{j}, & \rho_{j} \bar{\rho}_{\sigma(j)}=1, & j>K .
\end{array}
$$

Note that $\Delta g_{\sigma(j)}$ is independent of $\eta_{\sigma(j)}$. Inserting (4.32) into the righthand side of (4.30) and discarding terms containing $\bar{\xi}_{j}$ yields $\Delta g_{\sigma(j)}(\xi, \eta)=$ 0 , where $\eta$ is given by (4.32). This shows that the holomorphic function $\Delta g_{\sigma(j)}$ vanishes on $\mathcal{Q}$. Since $\mathcal{Q}$ contains a totally real submanifold in $\mathbb{C}^{2 n}$ of maximal dimension, then $\Delta g_{j}=0$, i.e., $\left[g_{j}^{*}-g_{j}\right]_{d}=0$ for $j \leq K$. Similarly, inserting (4.32) into the right hand side of (4.31) and discarding terms containing $\bar{\xi}_{\sigma(j)}$ yields $\left[g_{j}^{*}-g_{j}\right]_{d}=0$ for $K<j \leq n$. Now (4.30)-(4.31) 
read $\Delta f_{j}(\xi, \eta)=0$, in which $\eta$ is given by (4.32). This shows that $f_{j}^{*}=f_{j}$. Now formula (4.29) yields $\left[D_{j k}\right]_{d-1}=0$. Thus, under the normalizing condition (4.27) we obtain by induction that $\left[f_{j}\right]_{d}=\left[f_{j}^{*}\right]_{d}$ and $\left[g_{j}^{*}\right]_{d}=\left[g_{j}\right]_{d}$. Therefore, $\mu_{j}, m_{j}$ are uniquely determined by $M$.

Now, we can show that $\left|\lambda_{\sigma(j)}\right|=1 /\left|\lambda_{j}\right|$ and $m_{j}$ satisfy (4.26). Note that $M=\varphi(M)$ is also given by (4.23) with $\mu_{j}$ being replaced by $\left|\lambda_{j} \lambda_{\sigma(j)}\right|^{2} \mu_{j}$ and $F_{j}, G_{j}$ being replaced by

$$
\begin{gathered}
F_{j}^{*}(\xi, \eta)=\lambda_{j}^{-1} F_{j} \circ \varphi(\xi, \eta)=\xi_{j}+O(2), \\
G_{j}^{*}(\xi, \eta)=\lambda_{j} G_{j} \circ \varphi(\xi, \eta)=\eta_{j}+O(2),
\end{gathered}
$$

respectively. By the above uniqueness result, we have $\left|\lambda_{\sigma(j)} \lambda_{j}\right|=1$ and $F_{j}^{*} / G_{\sigma(j)}^{*}=m_{j}$, which gives us (4.26). The proof of the proposition is complete.

The above proposition leads us to the question: If a holomorphic symplectic mapping (1.1)-(1.2) admits $n$ meromorphic eigenfunctions (4.25), can it be put into its Birkhoff normal form by a convergent transformation?

The rest of this section and Section 5 are devoted to this eigenfunction problem.

Before we turn to the eigenfunction problem, we first remark that a holomorphic symplectic mapping of the form (1.1)-(1.2) satisfies $\left|\lambda_{\sigma(j)}\right|$. $\left|\lambda_{j}\right|=1$, when it admits the Levi-flat real analytic set (4.23). Although the existence of eigenfunctions (4.25) puts no extra restriction on the eigenvalues of the mapping, it restricts the higher order terms in the Birkhoff normal, as shown in the following.

TheOREm 4.6. - Let $\varphi$ be a holomorphic symplectic mapping defined by (1.1)-(1.2). Assume that $\varphi$ admits $n$ meromorphic eigenfunctions $m_{j}$ given by (4.25). Let $\Phi$ be a formal symplectic transformation such that

$$
\Phi \varphi \Phi^{-1}:(\xi, \eta) \rightarrow\left(\lambda_{j} \xi_{j} e^{H_{\zeta_{j}}(\zeta)}, \lambda_{j}^{-1} \eta_{j} e^{-H_{\zeta_{j}}(\zeta)}\right)+O(k) .
$$

Then

$$
m_{j} \circ \Phi^{-1}(\xi, \eta)=\frac{\xi_{j} \kappa_{j}(\xi \eta)+\widehat{f}_{j}(\xi, \eta)}{\eta_{\sigma(j)}+\widehat{g}_{\sigma(j)}(\xi, \eta)}, \quad \min \left\{\operatorname{ord} \widehat{f}_{j}, \operatorname{ord} \widehat{g}_{\sigma(j)}\right\} \geq k,
$$

where $\kappa_{j}$ are power series in $\xi_{1} \eta_{1}, \ldots, \xi_{n} \eta_{n}$ with $\kappa_{j}(0)=1$. Moreover, the formal power series $\omega_{1}, \ldots, \omega_{n}$ in the normal form (1.3) of $\varphi$ satisfy

$$
\omega_{j}+\omega_{\sigma(j)}=0 \text {. }
$$

TOME 51 (2001), FASCICULE 1 
Proof. - For brevity, we replace $m_{j} \circ \Phi^{-1}$ by $m_{j}$, and $\varphi$ by $\Phi \circ \varphi \circ \Phi^{-1}$. Put $m_{j}$ in the form (4.25), where $f_{j}, g_{j}$ are now formal power series with $g_{j}$ satisfying the normalizing condition (4.27).

¿From $m_{j} \circ \varphi=\lambda_{j} \lambda_{\sigma(j)} m_{j}$, we get

$$
\begin{aligned}
\left(\eta_{\sigma(j)}+\right. & \left.g_{\sigma(j)}\right)\left(\xi_{j} e^{\omega_{j}}+\lambda_{j}^{-1} f_{j} \circ \varphi\right) \\
& =\left(\xi_{j}+f_{j}\right)\left(\eta_{\sigma(j)} e^{-\omega_{\sigma(j)}}+\lambda_{\sigma(j)} g_{\sigma(j)} \circ \varphi\right)+O(k+1) .
\end{aligned}
$$

Assume for induction that

$$
\begin{array}{r}
f_{j}(\xi, \eta)=\xi_{j} \kappa_{j}(\xi \eta)+O(d), \quad g_{j}(\xi, \eta)=O(d), \\
\omega_{j}(\xi \eta)+\omega_{\sigma(j)}(\xi \eta)=O\left(|(\xi, \eta)|^{d-1}\right)
\end{array}
$$

hold for some $d<k-1$. We would like to show that the above identities hold if $d$ is replaced by $d+1$. Setting $\eta_{\sigma(j)}=0$ in (4.34), one gets from (4.35) and the normalizing condition (4.27) that

$$
g_{\sigma(j)}(\xi, \eta)=\lambda_{\sigma(j)} g_{\sigma(j)}\left(\lambda \xi, \lambda^{-1} \eta\right)+O(d+1) .
$$

Hence, $g_{j}(\xi, \eta)=O(d+1)$. Now, terms of order $d+1$ in (4.34) give us

$$
\xi_{j}\left[\omega_{j}+\omega_{\sigma(j)}\right]_{(d-1) / 2}(\xi \eta)+\lambda_{j}^{-1}\left[f_{j}\right]_{d}\left(\lambda \xi, \lambda^{-1} \eta\right)=\left[f_{j}\right]_{d}
$$

where $\left[\omega_{j}+\omega_{\sigma(j)}\right]_{(d-1) / 2}$ is treated as zero if $(d-1) / 2$ is not an integer. Set

$$
e_{j}=(0, \ldots, 1, \ldots, 0) \in \mathbb{Z}^{n},
$$

where 1 is the $j$-th entry. Collecting terms $\xi^{\alpha} \eta^{\beta}$ for $\alpha-\beta=e_{j}$ yields

$$
\left[\omega_{j}+\omega_{\sigma(j)}\right]_{(d-1) / 2}(\xi \eta)=0 .
$$

We now have $\left[f_{j}\right]_{d}\left(\lambda \xi, \lambda^{-1} \eta\right)=\lambda_{j}\left[f_{j}\right]_{d}(\xi, \eta)$. Therefore, $\left[f_{j}\right]_{d}(\xi, \eta)$ contains only terms $\xi^{\alpha} \eta^{\beta}$ with $\alpha-\beta=e_{j}$, and (4.35) hold if $d$ is replaced by $d+1$. By induction, (4.15) holds for $d=k$. This shows that $\min \left\{\right.$ ord $\hat{f}_{j}$, ord $\left.\hat{g}_{j}\right\} \geq k$, as stated in the theorem.

The identity (4.33) follows from the above result (with $k=\infty$ ) and the existence of $\Phi$ that normalizes $\varphi$. The proof of the theorem is complete.

As a consequence of Proposition 4.5 and Proposition 4.6, we know that if $\varphi$ has an invariant Levi-flat real analytic set defined by (4.21) with $\sigma^{d}=$ Id for some odd integer, then (4.33) implies that all $\omega_{j}=0$, that is that $\varphi$ is formally linearizable. By a theorem of Rüssmann [14], we obtain the following.

Corollary 4.7. - Let $\varphi$ be a holomorphic symplectic mapping defined by (1.1)-(1.2). Assume that $\varphi$ admits an invariant Levi-flat real 
analytic set given by (4.21) with $\sigma^{k}=$ Id for some odd integer $k$. If the eigenvalues $\lambda_{j}$ of $\varphi$ satisfy the Diophantine condition

$$
\left|\lambda^{\alpha}-\lambda_{j}\right| \geq c /|\alpha|^{\tau}, \quad|\alpha|>1, j=1, \ldots, n
$$

for some positive constant $c, \tau$, then $\varphi$ is linearizable by holomorphic symplectic mappings.

For $n=1$, see Proposition 4.2 with weaker hypotheses.

To state the next theorem, we need some notation. Let $\sigma$ be a permutation of $1, \ldots, n$, and let $m_{j}$, given by (4.25), be meromorphic eigenfunctions of $\varphi$. Theorem 4.6 implies that if $\Phi$ normalizes $\varphi$, then

$$
m_{j} \circ \Phi^{-1}(\xi, \eta)=\xi_{j} \kappa_{j}(\xi \eta) / \eta_{\sigma(j)} .
$$

For $1 \leq j \leq n$, recall that $\tau_{j}$ is the smallest positive integer with $\sigma^{\tau_{j}}(j)=j$. Define

$$
\tau_{j} \kappa=1, \quad \text { for } \tau_{j}=1 ; \quad \delta_{j} \kappa=\prod_{k=0}^{\tau_{j}-1} \kappa_{\sigma^{k}(j)}^{(-1)^{k}}, \quad \text { for } \tau_{j}>1 .
$$

One readily sees that

$$
\delta_{j} \kappa \cdot \delta_{\sigma(j)} \kappa= \begin{cases}1, & \text { if } \tau_{j} \text { is one or even, } \\ \kappa_{j}^{2}, & \text { otherwise. }\end{cases}
$$

The dependence of $\delta_{j} \kappa$ on the formal normalizing transformation $\Phi$ is described as the following.

Proposition 4.8. - Let $\delta_{1} \kappa, \ldots, \delta_{n} \kappa$ be as above. Fix $j$ with $1 \leq j \leq n$. The formal power series $\delta_{j} \kappa$ is independent of the choice of $\Phi$ if $\tau_{j}$ is even, but $\delta_{j} \kappa \equiv 1$ for some formal normalizing transformation $\Phi$ if $\tau_{j}$ is odd.

Proof. - Let $\tilde{\Phi}$ be another formal symplectic mapping which transforms $\varphi$ into (1.3). Put $m_{j} \circ \tilde{\Phi}^{-1}=\xi_{j} \tilde{\kappa}_{j} / \eta_{\sigma(j)}$. For $\left(\xi^{\prime}, \eta^{\prime}\right)=\tilde{\Phi} \circ \Phi^{-1}(\xi, \eta)$, one has

$$
\xi_{j}^{\prime} \tilde{\kappa}_{j}\left(\xi^{\prime} \eta^{\prime}\right) / \eta_{\sigma(j)}^{\prime}=\xi_{j} \kappa_{j} / \eta_{\sigma(j)}
$$

Since $\tilde{\Phi} \circ \Phi^{-1}$ preserves the normal form of $\varphi$, the formal theory of Birkhoff's normal form says that

$$
\tilde{\Phi} \circ \Phi^{-1}: \xi_{j}^{\prime}=\xi_{j} e^{G_{\zeta_{j}}(\xi \eta)}, \quad \eta_{j}^{\prime}=\eta_{j} e^{-G_{\zeta_{j}}(\xi \eta)}, \quad j=1, \ldots, n,
$$

where $G$ is a formal power series in $\zeta=\xi \eta$. Now (4.39) yields

$$
e^{G_{\zeta_{j}}(\zeta)+G_{\zeta_{\sigma(j)}}(\zeta)} \tilde{\kappa}_{j}(\zeta)=\kappa_{j}(\zeta),
$$

TOME 51 (2001), FASCICULE 1 
which gives us $\delta_{j} \tilde{\kappa}(\zeta)=\delta_{j} \kappa(\zeta)$ if $\tau_{j}$ is even. When $\tau_{j}$ is odd, we get

$$
e^{2 G_{\zeta_{j}}(\zeta)} \delta_{j} \tilde{\kappa}(\zeta)=\delta_{j} \kappa(\zeta)
$$

Solving the above equation for a formal power series $G$, one obtains a formal normalizing transformation $\tilde{\Phi}$ such that $\delta_{j} \tilde{\kappa} \equiv 1$. The proof of the proposition is complete.

We now state the following theorem.

THEOREM 4.9. - Let $\varphi, m_{1}, \ldots, m_{n}$ be as in Theorem 4.6, and let $\delta_{j} \kappa$ be the associated formal power series. Assume that $\sigma^{2}=\mathrm{Id}$, and that there exists a formal transformation $\Phi$ normalizing $\varphi$ such that all $\delta_{j} \kappa \circ \Phi$ are convergent. Then $\varphi$ is normalizable by holomorphic symplectic mappings.

In conclusion of this section, note that Theorem 1.2 follows from Proposition 4.5 and Theorems 4.6 and 4.9 .

\section{Normalization of holomorphic symplectic mappings with meromorphic eigenfunctions.}

Recall that Proposition 4.5 provides us the relationship between the Levi-flat invariant sets of the form (4.23) of a holomorphic symplectic mapping and meromorphic eigenfunctions of the mapping. The relationship between meromorphic eigenfunctions and Birkhoff normal forms of holomorphic symplectic mappings might be interesting in its own right.

In Section 4, we introduced the following eigenfunctions:

$$
m_{j}=\frac{\xi_{j}+f(\xi, \eta)}{\eta_{\sigma(j)}+g_{\sigma(j)}(\xi, \eta)}, \quad j=1, \ldots, n,
$$

where $\sigma$ is a permutation of $1, \ldots, n$, and $f_{j}(\xi, \eta)=O(3), g_{j}(\xi, \eta)=$ $O(3)$ are holomorphic. We should mention that we have no example of holomorphic symplectic mappings having eigenfunctions $m_{j}$, for which the Birkhoff normalization diverges.

The purpose of this section is to prove Theorem 4.9. However, we need a further reduction for the theorem, which is a necessary step for us to be able to apply the KAM method. Recall from Section 4 (Theorem 4.6) that if a formal holomorphic symplectic mapping $\Phi$ normalizes the symplectic mapping $\varphi$, then $m_{j} \circ \Phi^{-1}$ are of the form

$$
\xi_{j} \kappa_{j}(\xi \eta) / \eta_{\sigma(j)}, \quad j=1, \ldots, n .
$$


Assuming that $\sigma^{2}=$ Id and all $\delta_{j} \circ \Phi$ are convergent, we now modify $m_{j}$ such that

$$
\delta_{j} \kappa \equiv 1, \quad j=1, \ldots, n .
$$

If $\sigma(j)=j$, we simply put $\tilde{m}_{j}=m_{j}$. Otherwise, say $\sigma(1)=2$, we put

$$
\tilde{m}_{1}=m_{1} /\left(\delta_{1} \kappa \circ \Phi\right), \quad \tilde{m}_{2}=m_{2} \text {. }
$$

Then $\delta_{1} \tilde{m}=\tilde{m}_{1} / \tilde{m}_{2}=1=\delta_{2} \tilde{m}$. Let us still denote by $m_{j}$ the modified meromorphic functions $\tilde{m}_{j}$. In such a way we achieve (5.3).

Since $\sigma^{2}=\mathrm{Id}$, Proposition 4.8 implies that (5.3) remains true under all formal symplectic transformations that normalize $\varphi$. Also, (5.3) is equivalent to $\kappa_{j}=\kappa_{\sigma(j)}$, since $\delta_{j} \kappa=\kappa_{j} / \kappa_{\sigma(j)}$ when $\sigma^{2}=\mathrm{Id}$.

Thus, Theorem 4.9 is reduced to the following special case.

THEOREM 5.1. - Let $\varphi$ be a holomorphic symplectic mapping given by (1.1)-(1.2). Let $m_{1}, \ldots, m_{n}$ be $n$ meromorphic eigenfunctions of $\varphi$ of the form (5.1) with $\sigma^{2}=\mathrm{Id}$. Let $\Phi$ be a formal transformation that normalizes $\varphi$ and transforms $\kappa_{j}$ into (5.2). Assume that $\kappa_{\sigma(j)}=\kappa_{j}$ for $j=1, \ldots, n$. Then $\varphi$ can be transformed into its Birkhoff normal form by some holomorphic symplectic transformation.

We now proceed to prove Theorem 5.1, by applying the KAM method. Starting with the holomorphic symplectic mapping $\varphi$ defined by (1.1)-(1.2), we construct a sequence of holomorphic symplectic mappings $\phi_{m}$ as follows. Put $\varphi_{1}=\varphi$. Let $\Phi_{1}$ be the unique normalized formal transformation transforming $\varphi_{1}$ into its normal form. Let $\sum \lambda_{j} \xi_{j} \eta_{j}^{\prime}+H_{1}\left(\xi, \eta^{\prime}\right)$ be the generating function of $\varphi_{1}$, that is,

$$
\varphi_{1}: \xi_{j}^{\prime}=\lambda_{j} \xi_{j}+\partial_{\eta_{j}^{\prime}} H_{1}\left(\xi, \eta^{\prime}\right), \quad \eta_{j}^{\prime}=\lambda_{j}^{-1} \eta_{j}-\lambda_{j}^{-1} \partial_{\xi_{j}} H_{1}\left(\xi, \eta^{\prime}\right) .
$$

Let $\sum \xi_{j} \eta_{j}^{\prime}+S_{1}\left(\xi, \eta^{\prime}\right)$ be the generating function of $\Phi_{1}$. ¿From the formal theory of Birkhoff's normal form, one knows that ord $S_{1}=d_{0} \equiv \operatorname{ord} P H_{1}$ for

$$
P H_{1}\left(\xi, \eta^{\prime}\right)=\sum_{\alpha \neq \beta} H_{1, \alpha \beta} \xi^{\alpha} \eta^{\prime \beta} .
$$

Let $\phi_{1}$ be the holomorphic symplectic mapping generated by the truncated power series

$$
\tilde{S}_{1}\left(\xi, \eta^{\prime}\right)=\sum_{j} \xi_{j} \eta_{j}^{\prime}+\sum_{k=d_{0}}^{2 d_{0}-3}\left[S_{1}\right]_{k}\left(\xi, \eta^{\prime}\right)
$$

with

$$
\left[S_{1}\right]_{k}\left(\xi, \eta^{\prime}\right) \equiv \sum_{\alpha+\beta=k} S_{1, \alpha \beta} \xi^{\alpha} \eta^{\prime \beta} .
$$


Define $\varphi_{2}=\phi_{1} \circ \varphi_{1} \circ \phi_{1}^{-1}$. Repeating the above construction, we find holomorphic symplectic transformations $\varphi_{k}, \phi_{k}$ with $\varphi_{k+1}=\phi_{k} \circ \varphi_{k} \circ \phi_{k}^{-1}$. Let $\sum \lambda_{j} \xi_{j} \eta_{j}^{\prime}+H_{k}\left(\xi, \eta^{\prime}\right)$ be the generating function of $\varphi_{k}$ and put $d_{k}=$ ord $P H_{k}$.

If one of $d_{k}$ is infinite, proof of Theorem 5.1 is trivial; so we will assume that all $d_{k}$ are finite. Then

$$
d_{k+1} \geq 2 d_{k}-2, \quad d_{0} \geq 3 .
$$

In particular, $d_{k}-2 \geq 2^{k}, k=0,1, \ldots$ It is clear that as $k \rightarrow \infty$ the limit of $\phi_{k} \circ \phi_{2} \circ \cdots \circ \phi_{1}$ is a formal symplectic mapping $\phi$ such that $\phi \circ \varphi \circ \phi^{-1}$ is in the Birkhoff normal form. We shall complete the proof of Theorem 5.1 by showing the convergence of $\phi$.

To avoid functional equations involving small divisors, we shall determine $\phi_{1}$ through eigenfunctions $m_{j}$. Changing notations, replace $d_{0}, \tilde{S}_{1}$ and $\phi_{1}$ by $d, S, \phi$, respectively. We also rewrite

$$
m_{j}(\xi, \eta)=\frac{\xi_{j} \kappa_{j}(\xi \eta)+f_{j}(\xi, \eta)}{\eta_{\sigma(j)}+g_{\sigma(j)}(\xi, \eta)}
$$

where $f_{j}, g_{j}$ satisfy

$$
f_{j \alpha \beta}=0, \quad \alpha-\beta=e_{j} ; \quad g_{j \alpha \beta}=0, \quad \beta_{j} \neq 0,
$$

in which $e_{j}=(0, \ldots, 1, \ldots, 0)$. Theorem 4.6 implies that ord $f_{j}$, ord $g_{j} \geq$ $d-1$.

One knows that

$$
\phi: \xi_{j}^{\prime}=\xi_{j}+u_{j}(\xi, \eta), \quad \eta_{j}^{\prime}=\eta_{j}+v_{j}(\xi, \eta), \quad j=1, \ldots, n
$$

is determined by

$$
\xi_{j}^{\prime}=\xi_{j}-S_{\eta_{j}}\left(\xi, \eta^{\prime}\right), \quad \eta_{j}^{\prime}=\eta_{j}+S_{\xi_{j}}\left(\xi, \eta^{\prime}\right),
$$

where $S$, according to the normalizing condition on $\Phi$, satisfies

$$
S_{\alpha \alpha}=0, \quad \text { ord } S=d .
$$

One also has

$$
u_{j}(\xi, \eta)=-S_{\eta_{j}}(\xi, \eta)+O(2 d-3), \quad v_{j}(\xi, \eta)=S_{\xi_{j}}(\xi, \eta)+O(2 d-3) .
$$

Set

$$
m_{j}(\xi, \eta)=\hat{m}_{j} \circ \phi=\frac{\left(\xi_{j}+u_{j}\right) \hat{\kappa}_{j} \circ \phi(\xi, \eta)+\hat{f}_{j} \circ \phi(\xi, \eta)}{\eta_{\sigma(j)}+v_{\sigma(j)}+\hat{g}_{\sigma(j)} \circ \phi(\xi, \eta)}
$$

with $\hat{f}_{j}, \hat{g}_{j}$ satisfying (5.5). By Theorem 4.6, we have

$$
\text { ord } \hat{f}_{j}, \text { ord } \hat{g}_{j} \geq 2 d-3 ; \quad \operatorname{ord}\left(\hat{\kappa}_{j}-\kappa_{j}\right) \geq(d-2) / 2 \text {. }
$$


The linearized equations of (5.9) are

$$
\eta_{\sigma(j)} S_{\eta_{j}}(\xi, \eta)+\xi_{j} S_{\xi_{\sigma(j)}}(\xi, \eta)=p_{j}(\xi, \eta), \quad j=1, \ldots, n
$$

with

(5.11) $p_{j}=\xi_{j} \eta_{\sigma(j)} \nabla_{\zeta} \log \kappa_{j} \cdot\left(\xi S_{\xi}-\eta S_{\eta}\right)$

$$
+\xi_{j} g_{\sigma(j)}-\eta_{\sigma(j)} f_{j} / \kappa_{j}+\xi_{j} \eta_{\sigma(j)}\left(\hat{\kappa}_{j} / \kappa_{j}-1\right)+O(2 d-2) .
$$

In particular,

$$
\xi_{j} S_{\xi_{j}}+\eta_{j} S_{\eta_{j}}=p_{j}(\xi, \eta), \quad \text { for } \sigma(j)=j .
$$

For $\sigma(j) \neq j$, rewrite (5.10) as

$$
\xi_{j}^{-1} S_{\eta_{j}}+\eta_{\sigma(j)}^{-1} S_{\xi_{\sigma(j)}}=\nabla_{\zeta} \log \kappa_{j} \cdot\left(\xi S_{\xi}-\eta S_{\eta}\right)+\tilde{p}_{j}
$$

with

$$
\tilde{p}_{j}(\xi, \eta)=g_{\sigma(j)} / \eta_{\sigma(j)}-f_{j} /\left(\xi_{j} \kappa_{j}\right)+\hat{\kappa}_{j} / \kappa_{j}-1+\cdots,
$$

in which the omitted terms are of the form $\xi^{\alpha} \eta^{\beta} / \xi_{i} \eta_{\sigma(j)}$ with $|\alpha|+|\beta| \geq$ $2 d-2$. Using (5.3), we obtain

$$
\sum_{k=0}^{\tau_{j}-1}(-1)^{k}\left(\frac{S_{\eta_{\sigma^{k}(j)}}}{\xi_{\sigma^{k}(j)}}+\frac{S_{\xi_{\sigma^{k+1}(j)}}}{\eta_{\sigma^{k+1}(j)}}\right)=\sum_{k=0}^{\tau_{j}-1}(-1)^{k} \tilde{p}_{\sigma^{k}(j)} \equiv \Delta_{j} \tilde{p}
$$

in other words,

$$
\frac{S_{\eta_{j}}}{\xi_{j}}-(-1)^{\tau_{j}} \frac{S_{\xi_{j}}}{\eta_{j}}+\sum_{k=1}^{\tau_{j}-1}(-1)^{k}\left(\frac{S_{\eta_{\sigma^{k}(j)}}}{\xi_{\sigma^{k}(j)}}-\frac{S_{\xi_{\sigma^{k}(j)}}}{\eta_{\sigma^{k}(j)}}\right)=\Delta_{j} \tilde{p}
$$

for $\sigma(j) \neq j$.

LEMMA 5.2. - Let $\sum \xi_{j} \eta_{j}+S(\xi, \eta)$, satisfying (5.8), be the generating function of the unique formal mapping $\Phi$ that normalizes $\varphi$. Then $S \equiv[S]_{d}+\cdots+[S]_{2 d-3}$ is uniquely determined by (5.12) and (5.14).

Proof. - Let $S$ be the solution to (5.12) and (5.14), which satisfies the normalization condition (5.8). Fix $\alpha, \beta$ with $\alpha-\beta \neq 0$ and choose $j$ so that

$$
\left|\alpha_{j}-\beta_{j}\right|=\max \left\{\left|\alpha_{k}-\beta_{k}\right|\right\} .
$$

If $\sigma(j)=j$, then (5.12) implies that

$$
S_{\alpha \beta}=p_{j \alpha \beta} /\left(\alpha_{j}+\beta_{j}\right) .
$$

Assume now that $\sigma(j) \neq j$. To determine $S_{\alpha \beta}$, we need to consider all coefficients of $\xi^{\alpha^{\prime}} \eta^{\beta^{\prime}}$ in (5.14), for which

$$
\alpha^{\prime}-\beta^{\prime}=\alpha-\beta ; \quad \alpha_{k}^{\prime} \geq-1, \quad \beta_{k}^{\prime} \geq-1, \quad k=1, \ldots, n .
$$


Put $S_{\alpha \beta}=0$ for $\alpha_{k} \leq-1$ or $\beta_{k} \leq-1$. ¿From (5.14) we obtain

$$
\begin{array}{r}
\left(\beta_{j}^{\prime}-(-1)^{\tau_{j}} \alpha_{j}^{\prime}\right) S_{\alpha^{\prime}, \beta^{\prime}}+\sum_{k=1}^{\tau_{j}-1}(-1)^{k}\left(\beta_{\sigma^{k}(j)}-\alpha_{\sigma^{k}(j)}\right) S_{\alpha^{\prime}+e_{\sigma^{k}(j)}}-e_{j}, \beta^{\prime}+e_{\sigma^{k}(j)}-e_{j} \\
=\left(\Delta_{j} \tilde{p}\right)_{\alpha^{\prime}-e_{j}, \beta^{\prime}-e_{j}} .
\end{array}
$$

If $\alpha_{j}^{\prime}+\beta_{j}^{\prime}=\min \left\{\tilde{\alpha}_{j}+\tilde{\beta}_{j} \mid \tilde{\alpha}-\tilde{\beta}=\alpha-\beta\right\}$, then

$$
S_{\alpha^{\prime} \beta^{\prime}}=\frac{1}{\beta_{j}^{\prime}-(-1)^{\tau_{j}} \alpha_{j}^{\prime}} p_{j, \alpha^{\prime}-e_{j}, \beta^{\prime}-e_{j}} .
$$

Assume that $S_{\alpha^{\prime} \beta^{\prime}}$ have been determined for all $\alpha^{\prime}, \beta^{\prime}$ with $\alpha^{\prime}-\beta^{\prime}=\alpha-\beta$ and $\alpha_{j}^{\prime}+\beta_{j}^{\prime}<l$. Then for $\alpha^{\prime}, \beta^{\prime}$ with $\alpha_{j}^{\prime}+\beta_{j}^{\prime}=l$, we have

$$
\begin{aligned}
S_{\alpha^{\prime} \beta^{\prime}}= & \sum_{k=1}^{\tau_{j}-1}(-1)^{k-1} \frac{\beta_{\sigma^{k}(j)}-\alpha_{\sigma^{k}(j)}}{\beta_{j}^{\prime}-(-1)^{\tau_{j}} \alpha_{j}^{\prime}} S_{\alpha^{\prime}-e_{j}+e_{\sigma(j)}, \beta^{\prime}-e_{j}+e_{\sigma(j)}} \\
& +\frac{1}{\beta_{j}^{\prime}-(-1)^{\tau_{j}} \alpha_{j}^{\prime}}\left(\Delta_{j} \tilde{p}\right)_{\alpha^{\prime}-e_{j}, \beta^{\prime}-e_{j}} .
\end{aligned}
$$

Thus, (5.16)-(5.18) determine all $S_{\alpha^{\prime} \beta^{\prime}}$ for $\alpha^{\prime}-\beta^{\prime}=\alpha-\beta$. This completes the proof of the lemma.

We should point out that the proof of Lemma 5.2 does not depend on any assumption on $\delta_{j} \kappa$, and that the convergence of all $\delta_{j} \kappa$, as assumed in Theorem 4.9, would not give us an immediate good control of $S$ due to the recursive formulae (5.18) when some $\tau_{j} \geq 2$. To get estimates for $S$, it is crucial that all $\nabla_{\zeta} \log \delta_{j} \kappa$ vanish, which is obtained via (5.3) for the modified eigenfunctions. Even if $\delta_{j} \kappa \equiv 1$, the recursive formulae (5.18) lead to another problem: The radius of convergence would shrink by a constant factor of $\tau_{j}-1$ if $\tau_{j}>1$. Therefore, only for $\tau_{j}=1,2$ shall we have the control of the radius of convergence that is useful in iteration.

We need some notation. Let $f=\left(f_{1}, \ldots, f_{m}\right)$ be an $m$-tuple of holomorphic functions defined on $\Delta_{r} \subset \mathbb{C}^{n}$. Expand $f_{j}(z)=\sum f_{j \alpha} z^{\alpha}$ and let

$$
\begin{gathered}
f^{*}(z)=\sum_{\| \alpha \mid} f_{|\alpha|}^{*} z^{\alpha}, \quad f_{k}^{*}=\max \left\{\left|f_{j \alpha}\right| ;|\alpha|=k, j=1, \ldots, m\right\} ; \\
\|f\|_{r}=\sup \left\{\left|f_{j}(z)\right|: z \in \Delta_{r}, j=1, \ldots, m\right\} .
\end{gathered}
$$

In particular, one has $\xi^{*}=\xi_{j}^{*}=\sum\left(\xi_{j}+\eta_{j}\right)$, where $(\xi, \eta)$ are coordinates of $\mathbb{C}^{2 n}$. We also put

$$
(\nabla f)^{*}=\left(\partial_{z_{1}} f, \ldots, \partial_{z_{n}} f\right)^{*}, \quad\|\nabla f\|_{r}=\left\|\left(\partial_{z_{1}} f, \ldots, \partial_{z_{n}} f\right)\right\|_{r} .
$$

Given holomorphic functions $f$ and $g$, one has

$$
(f+g)^{*} \prec f^{*}+g^{*} ; \quad f^{*} \prec g^{*}, \quad \text { if } f \prec g .
$$


Also

$$
f^{*} \prec z^{*}(\nabla f)^{*}, \quad \text { if } f(0)=0 \text {. }
$$

Lemma 5.3. - Let $f$ be a holomorphic function defined on $\Delta_{r} \subset$ $\mathbb{C}^{n}$. Then $\left\|f^{*}\right\|_{(1-\theta) r} \leq\|f\|_{r} / \theta^{n+1}$.

Proof. — Since $\left|f_{\alpha}\right| \leq\|f\|_{r} / r^{|\alpha|}$, then $\left\|f^{*}\right\|_{(1-\theta) r}$ is bounded by

$$
\|f\|_{r} \sum_{\alpha}(1-\theta)^{|\alpha|}=\|f\|_{r} \sum_{k \geq 0}\left(\begin{array}{c}
n+k \\
n
\end{array}\right)(1-\theta)^{k}=\|f\|_{r} / \theta^{n+1} .
$$

This completes the proof of the lemma.

Lemma 5.4. - Let $\phi, S$ be given by (5.6)-(5.8). Assume that

$$
\|S\|_{r} \leq \theta^{2} r^{2} /(4 n), \quad 0<\theta<1 \text {. }
$$

Then

$$
\begin{aligned}
\phi, \phi^{-1}: \Delta_{(1-k \theta) r} & \rightarrow \Delta_{(1-(k-1) \theta) r}, \\
\left\|\phi^{-1}-\operatorname{Id}\right\|_{(1-k \theta) r} & \leq\|S\|_{(1-(k-2) \theta) r} /(r \theta), \quad k \geq 2 .
\end{aligned}
$$

Proof. — Fix $(\xi, \eta) \in \Delta_{(1-k \theta) r}$. Consider the mapping

$$
T: \xi_{j}^{\prime} \rightarrow \xi_{j}-S_{\eta_{j}^{\prime}}\left(\xi, \eta^{\prime}\right), \quad \eta_{j}^{\prime} \rightarrow \eta_{j}-S_{\xi_{j}}\left(\xi, \eta^{\prime}\right) .
$$

¿From (5.20) it follows that $T$ maps $\Delta_{(1-(k-1) \theta) r}$ into itself and

$$
\|\nabla T\|_{(1-\theta) r} \leq 1 /(4 n) .
$$

Hence, with the norm $\|(\xi, \eta)\|=\max \left\{\left|\xi_{j}\right|,\left|\eta_{j}\right|\right\}, T$ is a contraction map. By the fixed-point theorem, equations (5.7) determine a mapping $\phi: \Delta_{(1-k \theta) r} \rightarrow \Delta_{(1-(k-1) \theta) r}$. Applying the above argument to the mapping $\xi_{j} \rightarrow \xi_{j}^{\prime}+S_{\eta_{j}^{\prime}}\left(\xi, \eta^{\prime}\right), \eta_{j} \rightarrow \eta_{j}^{\prime}-S_{\xi_{j}}\left(\xi, \eta^{\prime}\right)$, one gets $\phi^{-1}: \Delta_{(1-k \theta) r} \rightarrow$ $\Delta_{(1-(k-1) \theta) r}$. Consequently, we have

$$
\left\|\phi^{-1}-\operatorname{Id}\right\|_{(1-k \theta) r} \leq\|\nabla S\|_{(1-(k-1) \theta) r} \leq\|S\|_{(1-(k-2) \theta) r} /(r \theta) .
$$

The proof of the lemma is complete.

Let $\kappa_{j}\left(\zeta_{1}, \ldots, \zeta_{n}\right)$ be as in (5.4). Put

$$
\kappa=\left(\kappa_{1}, \ldots, \kappa_{n}\right), \quad \kappa^{-1}=\left(\kappa_{1}^{-1}, \ldots, \kappa_{n}^{-1}\right) .
$$

LEMMA 5.5. - Let $\varphi$ and $S$ be as in Lemma 5.2, and let $\phi$ be given by (5.7). Assume that

$$
\left\|\left(\kappa^{-1}\right)^{*}\right\|_{r^{2}}<2, \quad r^{2}\left\|\left(\nabla_{\zeta} \kappa\right)^{*}\right\|_{r^{2}} \leq 1 /\left(16 n^{2}\right) .
$$

TOME 51 (2001), FASCICULE 1 
There exist constants $c_{1}, c_{2}>1$ such that if

$$
\|f\|_{r}+\|g\|_{r} \leq r \theta^{2 n+4} / c_{1}
$$

then

$$
\begin{aligned}
\phi, \phi^{-1}: \Delta_{(1-k \theta) r} & \rightarrow \Delta_{(1-(k-2) \theta) r}, \quad k \geq 2, \\
\left\|\phi^{-1}-\mathrm{Id}\right\|_{(1-2 \theta) r} & \leq c_{2}\left(\|f\|_{r}+\|g\|_{r}\right) / \theta^{2 n+3} .
\end{aligned}
$$

Proof. — We first introduce the following notation:

$$
\widehat{S}(\xi, \eta)=\sum\left|S_{\alpha \beta}\right| \xi^{\alpha} \eta^{\beta}
$$

which will be used only in the proof of this lemma. Fix $\alpha, \beta$ with $\alpha \neq \beta$ and let $j$ be defined by (5.15). Note that (5.15) implies that

$$
\left|\left\{\xi_{k} S_{\xi_{k}}-\eta_{k} S_{\eta_{k}}\right\}_{\alpha^{\prime} \beta^{\prime}}\right| \leq\left|\alpha_{j}-\beta_{j}\right| \widehat{S}_{\alpha^{\prime} \beta^{\prime}}, \quad k=1, \ldots, n
$$

for $\alpha^{\prime}-\beta^{\prime}=\alpha-\beta$. Hence, for $\sigma(j)=j$ one gets from (5.16) that

$$
\begin{aligned}
\left|S_{\alpha \beta}\right| & \leq\left\{\xi_{j} \eta_{j}\left(\nabla_{\zeta} \kappa\right)^{*}\left(\kappa^{-1}\right)^{*} \widehat{S}+\xi_{j} g_{j}^{*}+\eta_{j}\left(\kappa_{j}^{-1}\right)^{*}\left(f_{j}\right)^{*}\right\}_{\alpha \beta} \\
& \leq\left\{\xi^{*} \eta^{*}\left(\nabla_{\zeta} \kappa\right)^{*}\left(\kappa^{-1}\right)^{*} \widehat{S}+\xi^{*}\left(\kappa^{-1}\right)^{*}\left(f^{*}+g^{*}\right)\right\}_{\alpha \beta} .
\end{aligned}
$$

If $\sigma(j) \neq j,(5.17)-(5.18)$ yield

$$
\begin{aligned}
\left|S_{\alpha \beta}\right| & \leq\left(1+\min \left\{\alpha_{j}, \beta_{j}\right\}\right)\left\{\xi^{*} g^{*}+\eta^{*}\left(\kappa^{-1}\right)^{*} f^{*}\right\}_{\alpha \beta} \\
& \leq(|\alpha|+|\beta|)\left\{\xi^{*} g^{*}+\eta^{*}\left(\kappa^{-1}\right)^{*} f^{*}\right\}_{\alpha \beta} \\
& =\left\{\left(\xi \cdot \partial_{\xi}+\eta \cdot \partial_{\eta}\right)\left(\xi^{*}\left(\kappa^{-1}\right)^{*} f^{*}+\xi^{*} g^{*}\right)\right\}_{\alpha \beta} .
\end{aligned}
$$

Notice that we have used the essential assumption that $\tau_{j}=2$ to obtain the first inequality above. Thus,

$$
\widehat{S} \prec \frac{1}{1-\xi^{*} \eta^{*}\left(\nabla_{\zeta} \kappa\right)^{*}\left(\kappa^{-1}\right)^{*}}\left(\operatorname{Id}+\xi \partial_{\xi}+\eta \partial_{\eta}\right)\left(\xi^{*}\left(\kappa^{-1}\right)^{*}\left(f^{*}+g^{*}\right)\right) .
$$

¿From (5.19) we get $\left\|\xi^{*} \eta^{*}\left(\nabla_{\zeta} \kappa\right)^{*}\left(\kappa^{-1}\right)^{*}\right\|_{r}<1 / 2$ and

$$
\begin{aligned}
\left\|\left(\xi \cdot \partial_{\xi}+\eta \cdot \partial_{\eta}\right)\left(\xi^{*}\left(\kappa^{-1}\right)^{*}\left(f^{*}+g^{*}\right)\right)\right\|_{(1-\theta / 2) r} / \theta & \leq 8 n\left\|\xi^{*}\left(f^{*}+g^{*}\right)\right\|_{(1-\theta / 2) r} / \theta \\
& \leq \tilde{c}_{1} r\left(\|f\|_{r}+\|g\|_{r}\right) / \theta^{2 n+2}
\end{aligned}
$$

for some constant $\tilde{c}_{1} \cdot \gtreqless$ ¿From (5.25) it follows that

$$
\|\widehat{S}\|_{(1-\theta) r} \leq c_{1} r\left(\|f\|_{r}+\|g\|_{r}\right) /\left(4 n \theta^{2 n+2}\right) .
$$

Now, Lemma 5.4 implies that the mapping $\phi$, determined by $(5.6)-(5.7)$, satisfies (5.24), provided (5.22)-(5.23) hold.

The following is a special case of the Weierstrass preparation theorem. ANNALES DE L'INSTITUT FOURIER 
Lemma 5.6. - Let $f$ be a holomorphic function on $\Delta_{r} \subset \mathbb{C}^{2 n}$. Assume that $0<\theta<1 / 2$ and

$$
\|f\|_{r} \leq r \theta / 12, \quad f(\xi, \eta)=O(2) .
$$

Fix $1 \leq j \leq n$. There is a unique decomposition

$$
\eta_{j}+f(\xi, \eta)=(1+u(\xi, \eta))\left(\eta_{j}+g(\xi, \eta)\right), \quad u(0)=0
$$

where $u, g$ are holomorphic on $\Delta_{(1-\theta) r}$ with $g$ satisfying the normalizing condition (4.27). Moreover, for some constant $\tilde{c}_{2}>1$ one has

$$
\left\|g^{*}\right\|_{(1-\theta) r} \leq \tilde{c}_{2}\|f\|_{r} / \theta^{2 n+1}, \quad\left\|u^{*}\right\|_{(1-\theta) r} \leq \tilde{c}_{2}\|f\|_{r} /\left(r \theta^{2 n+2}\right) .
$$

Proof. - From (5.26) it follows that for $\xi_{j} \in \Delta_{r}$ there exists a unique solution $\eta_{j}=-g(\xi, \eta)$ to $\eta_{j}+f(\xi, \eta)=0$, given by

$$
\begin{aligned}
g(\xi, \eta) & =-\frac{1}{2 \pi i} \int_{\left|\eta_{j}\right|=r} \eta_{j} \frac{1+f_{\eta_{j}}(\xi, \eta)}{\eta_{j}+f(\xi, \eta)} d \eta_{j} \\
& =-\frac{1}{2 \pi i} \int_{\left|\eta_{j}\right|=r} \frac{\eta_{j} f_{\eta_{j}}(\xi, \eta)-f(\xi, \eta)}{\eta_{j}+f(\xi, \eta)} d \eta_{j}, \quad(\xi, \eta) \in \Delta_{r} \subset \Delta^{2 n-1} .
\end{aligned}
$$

Thus, Cauchy inequalities imply that

$$
\|g\|_{(1-\theta / 2) r} \leq 2\left(2\|f\|_{r} / \theta+\|f\|_{r}\right) \leq 6\|f\|_{r} / \theta
$$

Now the desired estimate for $g^{*}$ in (5.28) follows from Lemma 5.3.

To estimate $u^{*}$, note that $u=(f-g) /\left(\eta_{j}+g(\xi, \eta)\right)$. ¿From $(5.26)$ and (5.29) it follows that $\|u\|_{(1-\theta / 2) r} \leq 4\left(\|f\|_{r}+\|g\|_{(1-\theta / 2) r}\right) / r \leq 28\|f\|_{r} /(r \theta)$. In view of Lemma 5.3, we obtain (5.27). This completes the proof of the lemma.

Proposition 5.7. - Let $\phi$ be as in Lemma 5.5. Let $\hat{\kappa}_{j}, \hat{f}_{j}, \hat{g}_{j}$ be as in (5.9). There exist constants $c_{3}, c_{4}>1$ such that

$$
\begin{gathered}
\|\hat{f}\|_{(1-6 \theta) r}+\|\hat{g}\|_{(1-6 \theta) r} \leq c_{3}(1-\theta)^{d}\left(\|f\|_{r}+\|g\|_{r}\right) / \theta^{6 n+6} \\
\left\|\left(\nabla_{\zeta} \hat{\kappa}\right)^{*}\right\|_{((1-6 \theta) r)^{2}} \leq\left\|\left(\nabla_{\zeta} \kappa\right)^{*}\right\|_{r^{2}}+c_{3}\left(\|f\|_{r}+\|g\|_{r}\right) /\left(r^{3} \theta^{7 n+8}\right), \\
\left\|\left(\hat{\kappa}^{-1}\right)^{*}\right\|_{((1-6 \theta) r)^{2}} \leq\left\|\left(\hat{\kappa}^{-1}\right)^{*}\right\|_{r^{2}}+c_{3}\left(\|f\|_{r}+\|g\|_{r}\right) /\left(r \theta^{7 n+7}\right)
\end{gathered}
$$

provided (5.22) holds, and

$$
\|f\|_{r}+\|g\|_{r} \leq r \theta^{6 n+6} / c_{4}
$$

Proof. - Changing the notation in (5.6), we write $\phi^{-1}: \xi_{j}^{\prime}=\xi_{j}+u_{j}(\xi, \eta), \quad \eta_{j}^{\prime}=\eta_{j}+v_{j}(\xi, \eta)$.

TOME 51 (2001), FASCICULE 1 
We first want to express $\hat{f}_{j}, \hat{g}_{j}$ in terms of $f_{j}, g_{j}$ and $S$. Start with the decomposition

$$
\eta_{j}+v_{j}(\xi, \eta)+g_{j} \circ \phi^{-1}(\xi, \eta)=\left(1+a_{j}\right)\left(\eta_{j}+\hat{g}_{j}(\xi, \eta)\right) .
$$

¿From (5.24), one sees that

$$
\left\|v_{j}+g_{j} \circ \phi^{-1}\right\|_{(1-2 \theta) r} \leq 2 c_{2}\left(\|f\|_{r}+\|g\|_{r}\right) / \theta^{2 n+3} .
$$

In view of (5.26) and Lemma 5.6, we get from (5.28) that

$$
\begin{gathered}
\left\|a_{j}\right\|_{(1-3 \theta) r} \leq 2 c_{2} \tilde{c}_{2}\left(\|f\|_{r}+\|g\|_{r}\right) /\left((1-2 \theta) r \theta^{4 n+5}\right), \\
\left\|\hat{g}_{j}\right\|_{(1-3 \theta) r} \leq 2 c_{2} \tilde{c}_{2}\left(\|f\|_{r}+\|g\|_{r}\right) / \theta^{4 n+4}
\end{gathered}
$$

for $c_{4}>24 c_{2}$. Since ord $\hat{g}_{j} \geq 2 d-3 \geq d$, the Schwarz lemma yields

$$
\|\hat{g}\|_{(1-3 \theta) r} \leq 2 c_{2} \tilde{c}_{2}\left(\frac{1-4 \theta}{1-3 \theta}\right)^{d}\left(\|f\|_{r}+\|g\|_{r}\right) / \theta^{4 n+4} .
$$

For $0<\theta<1 / 6$ and $c_{4}>c_{2} \tilde{c}_{2},(5.32)-(5.33)$ imply that

$$
\left\|a_{j}\right\|_{(1-3 \theta) r}<1 / 2 \text {. }
$$

We now decompose

$$
\left(\left(\xi_{j}+u_{j}\right) \kappa_{j} \circ \phi^{-1}+f_{j} \circ \phi^{-1}\right) /\left(1+a_{\sigma(j)}\right)=\xi_{j} \hat{\kappa}_{j}+\hat{f}_{j} .
$$

Then

$$
\begin{aligned}
\xi_{j}\left(\hat{\kappa}_{j}-\kappa_{j}\right)+\hat{f}_{j}= & \left\{\xi_{j}\left(\kappa_{j} \circ \phi^{-1}-\kappa_{j}\right)+u_{j} \kappa_{j} \circ \phi^{-1}\right. \\
& \left.+f_{j} \circ \phi^{-1}-\xi_{j} \kappa_{j} a_{\sigma(j)}\right\} /\left(1+a_{\sigma(j)}\right) .
\end{aligned}
$$

We also have

$$
\begin{aligned}
(5.37)\left\|\kappa_{j} \circ \phi^{-1}-\kappa_{j}\right\|_{(1-2 \theta) r} & \leq 2 n r\left\|\nabla_{\zeta} \kappa_{j}\right\|_{((1-\theta) r)^{2}} \cdot\left\|\phi^{-1}-\mathrm{Id}\right\|_{(1-2 \theta) r} \\
& \leq c_{2}\left(\|f\|_{r}+\|g\|_{r}\right) /\left(r \theta^{2 n+3}\right),
\end{aligned}
$$

where the last inequality comes from (5.22) and (5.24). Using (5.33) and (5.37), one gets from (5.36) that

$$
\left\|\xi_{j}\left(\hat{\kappa}_{j}-\kappa_{j}\right)+\hat{f}_{j}\right\|_{(1-3 \theta) r} \leq c_{5}\left(\|f\|_{r}+\|g\|_{r}\right) / \theta^{4 n+5}
$$

for some constant $c_{5}>1$. Note that $\hat{f}_{j}^{*}$ and $\xi_{j}\left(\hat{\kappa}_{j}-\kappa_{j}\right)^{*}$ are majorized by $\left(\xi_{j}\left(\hat{\kappa}_{j}-\kappa_{j}\right)(\xi \eta)+\hat{f}_{j}(\xi, \eta)\right)^{*}$. Thus,

$$
\left\|\hat{f}^{*}\right\|_{(1-4 \theta) r} \leq c_{5}\left(\|f\|_{r}+\|g\|_{r}\right) / \theta^{6 n+6},
$$

$$
\left\|\left(\hat{\kappa}_{j}-\kappa_{j}\right)^{*}\right\|_{((1-4 \theta) r)^{2}} \leq c_{5}\left(\|f\|_{r}+\|g\|_{r}\right) /\left(r(1-4 \theta) \theta^{6 n+6}\right) .
$$


Since ord $\hat{f}_{j}$, ord $\hat{g}_{j} \geq d$, the Schwarz lemma gives

$$
\|\hat{f}\|_{(1-5 \theta) r} \leq c_{5}\left(\frac{1-5 \theta}{1-4 \theta}\right)^{d}\left(\|f\|_{r}+\|g\|_{r}\right) / \theta^{6 n+6} .
$$

Obviously, (5.34) and (5.39) yield (5.30) for $c_{3}>2 c_{2} \tilde{c}_{2}+c_{5}$. Note that $(1-\theta / 2)(1-4 \theta)^{2}>(1-5 \theta)^{2}$. Using (5.38) and Cauchy inequalities, we get

$$
\left\|\nabla_{\zeta}(\hat{\kappa}-\kappa)\right\|_{((1-5 \theta) r)^{2}} \leq \tilde{c}_{5}\left(\|f\|_{r}+\|g\|_{r}\right) /\left(r^{3} \theta^{6 n+7}\right) .
$$

In view of (5.19), we obtain

$$
\left\|\left(\nabla_{\zeta}(\hat{\kappa}-\kappa)\right)^{*}\right\|_{((1-6 \theta) r)^{2}} \leq \tilde{c}_{5}\left(\|f\|_{r}+\|g\|_{r}\right) /\left(r^{3} \theta^{7 n+8}\right)
$$

for some larger $\tilde{c}_{5}$. The above inequality yields the first half of (5.31) for $c_{3}>\tilde{c}_{5}$. For the second half of (5.31), note that

$$
\hat{\kappa}_{j}^{-1}-\kappa_{j}^{-1}=\kappa_{j} \sum_{k \geq 1}\left(\frac{\kappa_{j}-\hat{\kappa}_{j}}{\kappa_{j}}\right)^{k} .
$$

Thus, (5.38) and (5.32) yields

$$
\begin{aligned}
\left\|\left(\hat{\kappa}^{-1}-\kappa^{-1}\right)^{*}\right\|_{((1-6 \theta) r)^{2}} & \leq \frac{2}{\theta^{n+1}} \sum_{k \geq 1}\left(\frac{2 c_{5}\left(\|f\|_{r}+\|g\|_{r}\right)}{r(1-4 \theta) \theta^{6 n+6}}\right)^{k} \\
& \leq 8 c_{5}\left(\|f\|_{r}+\|g\|_{r}\right) /\left(r \theta^{7 n+7}\right) .
\end{aligned}
$$

This completes the proof of the proposition.

Put

$$
r_{k}=\frac{1}{2}\left(1+\frac{1}{k+1}\right) r, \quad k=0,1, \ldots
$$

Rewrite the above as

$$
r_{k+1}=\left(1-6 \theta_{k}\right) r_{k}, \quad \theta_{k}=\frac{1}{6(k+2)^{2}}, \quad k=0,1, \ldots
$$

Let us first prove a numerical result.

LEMMA 5.8. - Let $r_{k}, \theta_{k}$ be given as above, and let $0<r_{0}=r<1$. Let $b_{k}, B_{k}, K_{k}$ be nonnegative numbers satisfying

$$
\begin{gathered}
b_{k+1} \leq c_{3}\left(1-\theta_{k}\right)^{2^{k}} b_{k} / \theta_{k}^{6 n+6} \\
B_{k+1} \leq B_{k}+c_{3} b_{k} /\left(r_{k}^{3} \theta_{k}^{7 n+8}\right), \quad K_{k+1} \leq K_{k}+c_{3} b_{k} /\left(r_{k} \theta_{k}^{7 n+7}\right)
\end{gathered}
$$

for $k \geq 0$. Let $c_{4}$ be the constant given in Proposition 5.7. There exists $c_{6}>1$, independent of $r$, such that if

$$
b_{0} \leq r_{0} \theta_{0}^{6 n+6} / c_{6}, \quad r_{0}^{2} B_{0} \leq 1 /\left(32 n^{2}\right), \quad K_{0}<3 / 2,
$$

TOME 51 (2001), FASCICULE 1 
then for all $k>0$

$$
b_{k} \leq r_{k} \theta_{k}^{6 n+6} / c_{4}, \quad r_{k}^{2} B_{k} \leq 1 /\left(16 n^{2}\right), \quad K_{k}<2 .
$$

$$
\begin{aligned}
& \text { Proof. - Put } \\
& \qquad \hat{b}_{k}=r_{k} \theta_{k}^{7 n+9} /\left(32 n^{2} c_{3} c_{4}\right), \quad k=0,1, \ldots .
\end{aligned}
$$

It is easy to see that

$$
\hat{b}_{k+1} / \hat{b}_{k}=\left(1-6 \theta_{k}\right) \theta_{k+1}^{7 n+9} / \theta_{k}^{7 n+9} \geq\left(1-6 \theta_{0}\right) \theta_{1}^{7 n+9} / \theta_{0}^{7 n+9} \equiv c_{7} .
$$

On the other hand, one has

$$
b_{k+1} / b_{k} \leq c_{3}\left(1-\theta_{k}\right)^{2^{k}} / \theta_{k}^{6 n+6} \rightarrow 0, \quad k \rightarrow \infty .
$$

Hence, there exists $N$, independent of $r$, such that

$$
b_{k+1} / b_{k} \leq \hat{b}_{k+1} / \hat{b}_{k}, \quad k \geq N .
$$

Set

$$
c_{8}=1+\max _{k \leq N} c_{3}\left(1-\theta_{k}\right)^{2^{k}} / \theta_{k}^{6 n+6} .
$$

Then (5.42) implies that

$$
b_{k} \leq b_{0} c_{8}^{N} \leq r_{0} \theta_{0}^{6 n+6} c_{8}^{N} / c_{6}, \quad k \leq N .
$$

This shows that for sufficiently large $c_{6}$, one has $b_{k} \leq \hat{b}_{k}, k=0, \ldots, N$. Now (5.44) implies that $b_{k} \leq \hat{b}_{k}$ for all $k$. In particular, the estimates (5.43) hold for $b_{k}$. Also,

$$
r_{k+1}^{2} B_{k+1} \leq r_{0}^{2} B_{0}+\sum_{j=1}^{k} \frac{c_{3} b_{j}}{r_{j} \theta_{j}^{7 n+8}} \leq 1 /\left(32 n^{2}\right)+\sum_{j=1}^{\infty} \theta_{j} /\left(32 n^{2}\right)<1 /\left(16 n^{2}\right)
$$

and $K_{k+1} \leq K_{0}+\sum_{j=1}^{k} c_{3} b_{j} / r_{j} \theta_{j}^{7 n+7}<2$. This completes the proof of the lemma.

We now complete the proof of Theorem 4.9 as follows. With the notation introduced at the beginning of this section, we would like to show the convergence of a subsequence of $\phi_{1}^{-1} \circ \phi_{2}^{-1} \circ \cdots \circ \phi_{k}^{-1}$. To this end, it suffices to show that the sequence is bounded, or that

$$
\phi_{k}^{-1}: \Delta_{r_{k}} \rightarrow \Delta_{r_{k-1}}, \quad k=1,2, \ldots
$$

are well-defined for suitable $r_{0}=r$.

Start with

$$
m_{j}^{(1)}(\xi, \eta)=m_{j}(\xi, \eta)=\frac{\xi_{j} \kappa_{j}^{(1)}(\xi \eta)+f_{j}^{(1)}(\xi, \eta)}{\eta_{\sigma(j)}+g_{\sigma(j)}^{(1)}(\xi, \eta)} .
$$


Set

$$
b_{0}=\left\|f^{(1)}\right\|_{r_{0}}+\left\|g^{(1)}\right\|_{r_{0}}, B_{0}=\left\|\left(\nabla_{\zeta} \kappa^{(1)}\right)^{*}\right\|_{r_{0}^{2}}, K_{0}=\left\|\left(\left(\kappa^{(1)}\right)^{-1}\right)^{*}\right\|_{r_{0}^{2}} .
$$

Choose $r_{0}=r$ so small that initial condition (5.42) holds. Applying Lemma 5.5 to $\varphi_{1}$ and $\phi_{1}$ gives us $(5.45)$ for $\phi_{1}$. Put $m_{j}^{(2)}=m_{j}^{(1)} \circ \phi_{1}^{-1}$ and

$$
b_{1}=\left\|f^{(2)}\right\|_{r_{1}}+\left\|g^{(2)}\right\|_{r_{1}}, B_{1}=\left\|\left(\nabla_{\zeta} \kappa^{(2)}\right)^{*}\right\|_{r_{1}^{2}}, K_{1}=\left\|\left(\left(\kappa^{(2)}\right)^{-1}\right)^{*}\right\|_{r_{1}^{2}} .
$$

Since $d_{k}>2^{k}$, Proposition 5.7 says that inequality (5.41) holds for $k=1$. Consequently, Lemma 5.8 implies that the initial condition (5.43) holds for $k=1$. Recursively, one sees that the mappings (5.45) are well-defined for all $k$. This completes the proof of Theorem 4.9 .

\section{Hamiltonian vector fields.}

This section is to apply results for holomorphic symplectic mappings obtained in previous sections to holomorphic Hamiltonian systems.

Consider a holomorphic Hamiltonian function

$$
H(\xi, \eta)=\sum \lambda_{j} \xi_{j} \eta_{j}+h(\xi, \eta), \quad h(\xi, \eta)=O(3) .
$$

The corresponding Hamiltonian system of $H$ is

$$
d \xi_{j} / d t=\lambda_{j} \xi_{j}+H_{\eta_{j}}, \quad d \eta_{j} / d t=-\lambda_{j} \eta_{j}-H_{\xi_{j}}, \quad j=1, \ldots, n .
$$

The formal Birkhoff normal form of $H$ is then given by

$$
\widehat{H}(\xi, \eta)=\sum \lambda_{j} \xi_{j} \eta_{j}+\hat{h}(\xi \eta)
$$

where $\hat{h}$ is a formal power series in products $\xi_{1} \eta_{1}, \ldots, \xi_{n} \eta_{n}$, provided $\lambda=\left(\lambda_{1}, \ldots, \lambda_{n}\right)$ satisfies the non-resonance condition

$$
\alpha \cdot \lambda=\alpha_{1} \lambda_{1}+\cdots+\alpha_{n} \lambda_{n} \neq \lambda_{j}, \quad \alpha \in \mathbb{Z}^{n}
$$

for all $\alpha \neq e_{j}=(0, \ldots, 1, \ldots, 0)$. The formal flow of the Hamiltonian (6.3) is given by

$$
\widehat{\varphi}_{t}: \xi_{j}^{\prime}=\xi_{j} e^{t \widehat{H}_{\zeta_{j}}}, \quad \eta_{j}^{\prime}=\eta_{j} e^{-t \widehat{H}_{\zeta_{j}}}, \quad j=1, \ldots, n
$$

for $t \in \mathbb{C}$. We say that a real or complex time $t$ is non-exceptional with respect to $\lambda_{1}, \ldots, \lambda_{n}$, if the eigenvalues $e^{t \lambda_{1}}, \ldots, e^{t \lambda_{n}}$ satisfy the nonresonance condition (1.2). Note that, given $\lambda_{1}, \ldots, \lambda_{n}$ satisfying (6.4), the exceptional $t$ values are countable. 
We need the following.

Proposition 6.1. - Let $H$ be the Hamiltonian function (6.1) with $\lambda_{j}$ satisfying (6.4), and let $\varphi_{t}$ be its Hamiltonian flow. Assume that $t$ is nonexceptional. If $\varphi_{t}$ is in its Birkhoff normal form, so is $H$.

Proof. - Replacing $H$ by $t H$, one may assume that $t=1$. We need to prove that $H$ is a function of $\xi_{1} \eta_{1}, \ldots, \xi_{n} \eta_{n}$. Put

$$
H(x, y)=\tilde{H}\left(\xi_{1} \eta_{1}, \ldots, \xi_{n} \eta_{n}\right)+H_{k}(\xi, \eta),
$$

where $H_{k}(\xi, \eta)=O(k+1)$, and all terms in the power series expansion of $\tilde{H}$ have order at most $k$ in $\xi, \eta$. Note that for two holomorphic vector fields $v(\xi, \eta), \tilde{v}(\xi, \eta)$ with $v(0)=\tilde{v}(0)=0$ and $\tilde{v}(\xi, \eta)-v(\xi, \eta)=O(k)$, the corresponding flows $\varphi_{t}$ and $\tilde{\varphi}_{t}$ satisfy

$$
\tilde{\varphi}_{t}(\xi, \eta)-\varphi_{t}(\xi, \eta)=O\left(|(\xi, \eta)|^{k}\right)
$$

uniformly for $|t|<T<\infty$. The flow $\varphi_{t}$ of $(6.5)$ is of the form

$$
\begin{aligned}
& \xi_{j}(t)=\xi_{j} e^{t \tilde{H}_{\zeta_{j}}(\xi \eta)}+\sum a_{j \alpha \beta}(t) \xi^{\alpha} \eta^{\beta}, \\
& \eta_{j}(t)=\eta_{j} e^{-t \tilde{H}_{\zeta_{j}}(\xi \eta)}+\sum b_{j \alpha \beta}(t) \xi^{\alpha} \eta^{\beta}
\end{aligned}
$$

with

$$
a_{j \alpha \beta}(t)=b_{j \alpha \beta}(t) \equiv 0, \quad|\alpha|+|\beta|<k .
$$

For $\alpha+\beta=k$, one gets

$$
d a_{j \alpha \beta} / d t-\lambda_{j} a_{j \alpha \beta}=\left(\beta_{j}+1\right) H_{\alpha, \beta+e_{j}} e^{(\alpha-\beta) \cdot \lambda t}, \quad \alpha-\beta \neq e_{j}
$$

with $a_{j \alpha \beta}(0)=0$. Hence

$$
a_{j \alpha \beta}(t)=\left(\beta_{j}+1\right) \frac{e^{(\alpha-\beta) \cdot \lambda t}-e^{\lambda_{j} t}}{(\alpha-\beta) \cdot \lambda-\lambda_{j}} H_{\alpha, \beta+e_{j}}
$$

for $|\alpha|+|\beta|=k, \alpha-\beta \neq e_{j}$. Since $\varphi_{1}$ is in the normal form, then $a_{j \alpha \beta}(1)$ vanish for $\alpha-\beta \neq e_{j}$ and $|\alpha|+|\beta|=k$. Hence, $H_{\alpha \beta}=0$ for $\alpha \neq \beta,|\alpha|+|\beta|=k+1$, and $\beta \neq 0$. Computing the coefficients of $b_{j\left(\alpha-e_{j}\right) 0}$ yields $H_{\alpha 0}=0$ for $|\alpha|=k+1$. Thus, $[H]_{k+1}$ is a power series in $\xi_{1} \eta_{1}, \ldots, \xi_{n} \eta_{n}$. By induction, $H$ is a power series in $\xi_{1} \eta_{1}, \ldots, \xi_{n} \eta_{n}$. The proof of the proposition is complete.

With the above observation, we now introduce a notion. Let $M$ be the germ of a set at $0 \in \mathbb{C}^{2 n}$. We say that the germ $M$ is weakly invariant under the flow $\varphi_{t}$, if the germ $\varphi_{t}(M)$ is contained in $M$ for some non-exceptional $t \in \mathbb{C}$. From Theorems 1.1 and 4.1 and Proposition 6.1 , we obtain the following. 
Corollary 6.2. - Let $\varphi_{t}$ be a holomorphic Hamiltonian flow defined by (6.2) and (6.4), and let $M$ be a Levi-flat real analytic set defined by (1.4). Assume that $M$ is weakly invariant under the flow $\varphi_{t}$. Then the Hamiltonian system (6.2) is normalizable by convergent holomorphic symplectic mappings. Moreover, $M$ is invariant under $\varphi_{t}$ for all $t \in \mathbb{C}$.

¿From Theorem 4.6, we have the following.

Corollary 6.3. - Let $M$ be a Levi-flat analytic set defined by (1.5), and let $\varphi_{t}$ be the holomorphic Hamiltonian flow of (6.2) and (6.4). If $M$ is invariant under $\varphi_{t_{0}}$ for some non-exceptional $t_{0}$, then $M$ is invariant under $\varphi_{s t_{0}}$ for all $s \in \mathbb{R}$.

Proof. - By Theorem 3.6, $M$ is actually given by $r_{1}=\ldots=$ $r_{n}=0$ with $r_{j}$ being given by (4.23). Let $m_{j}=F_{j}(\xi, \eta) / G_{\sigma(j)}(\xi, \eta)$ be the meromorphic functions defined by (4.25). Take a formal symplectic transformation $\Phi=\operatorname{Id}+O(2)$ which transforms the Hamiltonian (6.1) into the Birkhoff normal form. Fix a non-exceptional $t_{0}$ such that $\varphi_{t_{0}}(M)=M$. By Theorem 4.6, we know that $m_{j} \circ \Phi^{-1}$ is an eigenfunction of the flow $\Phi \circ \varphi_{t} \circ \Phi^{-1}$ with eigenvalue $e^{t\left(\lambda_{j}+\lambda_{\sigma(j)}\right)}$. Hence, $m_{j}$ is also an eigenfunction of $\varphi_{t}$ with the same eigenvalue. Thus, $F_{j} \circ \varphi_{t}=u_{j} e^{t \lambda_{j}} F_{j}, G_{\sigma(j)} \circ \varphi_{t}=$ $u_{j} e^{-t \lambda_{\sigma(j)}} G_{\sigma(j)}$, where $u_{j}=1+O(1)$ are holomorphic functions dependent of $t$. Note that $\left|e^{t \lambda_{j}}\right|=\left|e^{-t \lambda_{\sigma(j)}}\right|$ hold for $t=t_{0}$, and hence holds for all $t=s t_{0}$ when $s$ is real. Now, it is straightforward that for each real $s$, $r_{j} \circ \varphi_{s t_{0}}=v_{j} r_{j}$ for some $v_{j} \neq 0$. In particular, $M$ is invariant under $\varphi_{s t_{0}}$. This completes the proof of the corollary.

Finally, Theorem 1.2 gives us the following.

Corollary 6.4. - Let $M$ be as in Theorem 1.2, and let $\varphi_{t}$ be as in Corollary 6.3. Assume that $M$ is weakly invariant under the flow $\varphi_{t}$. The Hamiltonian system (6.2) is normalizable by holomorphic symplectic mappings.

\section{Appendix: Birkhoff normal forms in terms of time-one mappings of formal Hamiltonian systems and formal generating functions.}

There are two ways to formulate the Birkhoff normal form for holomorphic symplectic mappings, of which one is of the form (1.3). The 
other normal form can be described in terms of generating functions. For the sake of the reader, we derive one normal form from another in this appendix.

Let $\left(\xi^{\prime}, \eta^{\prime}\right)=\varphi(\xi, \eta)$ be a holomorphic symplectic mapping (1.1). Regarding the mapping as a graph over $\xi, \eta^{\prime}$, on which the 1 -form $\sum\left(\eta_{j}^{\prime} d \xi_{j}+\right.$ $\left.\xi_{j}^{\prime} d \eta_{j}^{\prime}\right)$ is closed, one has

$$
\xi_{j}^{\prime}=\lambda_{j} \xi_{j}+S_{\eta_{j}^{\prime}}\left(\xi, \eta^{\prime}\right), \quad \eta_{j}=\lambda_{j} \eta_{j}^{\prime}+S_{\xi_{j}}\left(\xi, \eta^{\prime}\right)
$$

for some convergent power series $S\left(\xi, \eta^{\prime}\right)=O(3)$. Assume that $\lambda_{j}$ satisfy the non-resonance condition (1.2). By comparing the coefficients as in the Hamiltonian case (see [4], p. 85), there is a formal power series $T\left(\xi, \eta^{\prime}\right)=O(3)$ such that for the formal symplectic mapping $\Phi$ determined by

$$
\xi_{j}^{\prime}=\xi_{j}+T_{\eta_{j}^{\prime}}\left(\xi, \eta^{\prime}\right), \quad \eta_{j}=\eta_{j}^{\prime}+T_{\xi_{j}}\left(\xi, \eta^{\prime}\right),
$$

$\hat{\varphi}=\Phi \circ \varphi \circ \Phi^{-1}$ is of the form (7.1) with

$$
S=\hat{S}\left(\xi \eta^{\prime}\right)
$$

being a formal power series in the products $\zeta_{j}=\xi_{j} \eta_{j}^{\prime}$. Thus, the formal mapping given by (7.1)-(7.2) can also be referred as the Birkhoff normal form of $\varphi$.

Next, we want to put the formal mapping (7.1)-(7.2) into the form (1.3). From (7.1)-(7.2), one has

$$
\xi_{j}^{\prime}=\lambda_{j} \xi_{j}\left(1+\lambda_{j}^{-1} \hat{S}_{\zeta_{j}}\left(\xi \eta^{\prime}\right)\right), \quad \eta_{j}^{\prime}=\lambda_{j}^{-1} \eta_{j}\left(1+\lambda_{j}^{-1} \hat{S}_{\zeta_{j}}\left(\xi \eta^{\prime}\right)\right)^{-1}
$$

for $j=1, \ldots, n$. Hence, $\xi_{j} \eta_{j}$ are invariant by $\hat{\varphi}$. Solving for $\eta_{j}^{\prime}$ from the last $n$ equations above, one sees that

$$
\xi_{j}^{\prime}=\lambda_{j} \xi_{j} e^{G_{j}(\xi \eta)}, \quad \eta_{j}^{\prime}=\lambda_{j}^{-1} \eta_{j} e^{-G_{j}(\xi \eta)}
$$

for some formal power series $G_{j}(\xi \eta)$ with $G_{j}(0)=0$. Note that $G_{j}$ are determined by

$$
e^{G_{j}(\zeta)}=1+\lambda_{j}^{-1} \hat{S}_{\zeta_{j}}\left(\lambda^{-1} \zeta e^{G(\zeta)}\right), \quad j=1, \ldots, n,
$$

where $\lambda^{-1} \zeta e^{G(\zeta)} \equiv\left(\lambda_{1}^{-1} \zeta_{1} e^{G_{1}(\zeta)}, \ldots, \lambda_{n}^{-1} \zeta_{n} e^{G_{n}(\zeta)}\right)$. On the other hand, (7.3) yields

$$
\sum d \xi_{j}^{\prime} \wedge d \eta_{j}^{\prime}-\sum d \xi_{j} \wedge d \eta_{j}=d \sum G_{j}\left(\xi_{j} \eta_{j}\right) d\left(\xi_{j} \eta_{j}\right)
$$

The left-hand side vanishes. Hence, $G_{j}=H_{\zeta_{j}}$ for some formal power series $H$ in $\zeta$. 
Conversely, if $\left(\xi^{\prime}, \eta^{\prime}\right)=\hat{\varphi}(\xi, \eta)$ is given by (1.3), then $\hat{\varphi}$ preserves the products $\xi_{j} \eta_{j}$ and is of the form

$$
\xi_{j}^{\prime}=\lambda_{j} \xi_{j}\left(1+K_{j}\left(\xi \eta^{\prime}\right)\right), \quad \eta_{j}=\lambda_{j} \eta_{j}^{\prime}\left(1+K_{j}\left(\xi \eta^{\prime}\right)\right)
$$

with

$$
1+K_{j}(\zeta)=e^{\omega_{j}(\lambda \zeta K(\zeta))}, \quad j=1, \ldots, n
$$

Now, one has

$$
\sum \xi_{j}^{\prime} d \eta_{j}^{\prime}+\eta_{j} d \xi_{j}=d\left(\sum \lambda_{j} \xi_{j} \eta_{j}^{\prime}\right)+\sum \lambda_{j} K_{j}\left(\xi \eta^{\prime}\right) d\left(\xi_{j} \eta_{j}^{\prime}\right) .
$$

Hence, the last summand is closed, from which it follows that $K_{j}=$ $\lambda_{j}^{-1} \hat{S}_{\zeta_{j}}(\zeta)$ for some formal power series $\hat{S}$. This shows that $\hat{\varphi}$ is of the form (7.1)-(7.2). From (7.4)-(7.5), one can also see that the convergence of $\hat{S}$ implies the convergence of $H$, and vice versa.

Therefore, two normal forms (1.3) and (7.1)-(7.2) are equivalent, i.e., if one of the normal forms is realized by a holomorphic symplectic transformation, so is the other.

\section{BIBLIOGRAPHY}

[1] M. Artin, On the solutions of analytic equations, Invent. Math., 5 (1968), 277-291.

[2] E. BEDFORD, Holomorphic continuation of smooth functions over Levi-flat hypersurfaces, Trans. Amer. Math. Soc., 232 (1977), 323-341.

[3] G.D. Birkhoff, Surface transformations and their dynamical applications, Acta Math., 43 (1920), 1-119.

[4] G.D. Birkhoff, Dynamical Systems, AMS Coll. Publ., vol. 9, 1927, reprinted 1966.

[5] F. Bruhat and H. Cartan, Sur la structure des sous-ensembles analytiques réels, C. R. Acad. Sci. Paris, 244 (1957), 988-991.

[6] D. Burns and X. Gong, Singular Levi-flat real analytic hypersurfaces, Amer. J. Math., 121, no 1 (1999), 23-53.

[7] H. CARTAN, Variétés analytiques réelles et variétés analytiques complexes, Bull. Soc. Math. France, 85 (1957), 77-99.

[8] K. Diederich and J.E. Fornaess, Pseudoconvex domains with real-analytic boundary, Ann. Math. (2), 107, $\mathrm{n}^{\circ} 2$ (1978), 371-384.

[9] L.H. Eliasson, Normal forms for Hamiltonian systems with Poisson commuting integrals-elliptic case, Comment. Math. Helv., 65, $\mathrm{n}^{\circ} 1$ (1990), 4-35.

[10] Н. Іто, Convergence of Birkhoff normal forms for integrable systems, Comment. Math. Helv., 64 (1989), 412-461.

[11] J.K. Moser, The analytic invariants of an area-preserving mapping near a hyperbolic fixed point, Comm. Pure Appl. Math., 9 (1956), 673-692. 
[12] H. RÜSSMANN, Über die Existenz einer Normalform inhaltstreuer elliptischer Transformationen, Math. Ann., 137 (1959), 64-77.

[13] H. RÜssmann, Über das Verhalten analytischer Hamiltonscher Differentialgleichungen in der Nähe einer Gleichgewichtslösung, Math. Ann., 154 (1964), 285300 .

[14] H. RÜssmann, Über die Normalform analytischer Hamiltonscher Differentialgleichungen in der Nähe einer Gleichgewichtslösung, Math. Ann., 169 (1967), 55-72.

[15] B. SEgRe, Intorno al problem di Poincaré della rappresentazione pseudo-conform, Rend. Acc. Lincei, 13 (1931), 676-683.

[16] C.L. Siegel, On integrals of canonical systems, Ann. Math., 42 (1941), 806-822.

[17] C.L. Siegel, Über die Existenz einer Normalform analytischer Hamiltonscher Differntialgleichungen in der Nähe einer Gleichgewichtslösung, Math. Ann., 128 (1954), 144-170.

[18] J. VEy, Sur certains systèmes dynamiques séparables, Amer. J. Math., 100 (1978), $591-614$.

[19] S.M. Webster, On the mapping problem for algebraic real hypersurfaces, Invent. Math., $43, \mathrm{n}^{\circ} 1$ (1977), 53-68.

Manuscrit reçu le 11 avril 2000, accepté le 26 juillet 2000.

Xianghong GONG,

Oklahoma State University

Department of Mathematics Stillwater, OK 74078 (USA).

Current address:

University of Wisconsin - Madison

Department of Mathematics

Madison, WI 53706 (USA).

gong@math.wisc.edu 